University of Louisville

ThinkIR: The University of Louisville's Institutional Repository

$12-2011$

\title{
Theoretical and experimental studies on manipulation of fluorescence by gold nanoparticle : application for molecular imaging.
}

Jianting Wang 1983-

University of Louisville

Follow this and additional works at: https://ir.library.louisville.edu/etd

\section{Recommended Citation}

Wang, Jianting 1983-, "Theoretical and experimental studies on manipulation of fluorescence by gold nanoparticle : application for molecular imaging." (2011). Electronic Theses and Dissertations. Paper 1512.

https://doi.org/10.18297/etd/1512

This Doctoral Dissertation is brought to you for free and open access by ThinkIR: The University of Louisville's Institutional Repository. It has been accepted for inclusion in Electronic Theses and Dissertations by an authorized administrator of ThinkIR: The University of Louisville's Institutional Repository. This title appears here courtesy of the author, who has retained all other copyrights. For more information, please contact thinkir@louisville.edu. 


\title{
THEORETICAL AND EXPERIMENTAL STUDIES ON MANIPULATION OF FLUORESCENCE BY GOLD NANOPARTICLE: APPLICATION FOR MOLECULAR IMAGING
}

\author{
By \\ Jianting Wang \\ B.S., Tsinghua University, China, 2003 \\ M.S., Tsinghua University, China, 2006

\begin{abstract}
A Dissertation
Submitted to the Faculty of the

Graduate School of the University of Louisville in Partial Fulfillment of the Requirements for the Degree of
\end{abstract} \\ Doctor of Philosophy \\ Department of Chemical Engineering \\ University of Louisville \\ Louisville, Kentucky
}

December 2011 


\title{
THEORETICAL AND EXPERIMENTAL STUDIES ON
} MANIPULATION OF FLUORESCENCE BY GOLD NANOPARTICLE: APPLICATION FOR MOLECULAR IMAGING

\author{
By
}

Jianting Wang

B.S., Tsinghua University, China, 2003

M.S., Tsinghua University, China, 2006

A Dissertation Approved on

December 01, 2011

by the Following Committee:

Dissertation Director, Kyung A. Kang, Ph.D.

Paula J. Bates, Ph.D.

Michael H. Nantz. Ph.D.

Thomas L. Starr, Ph.D.

Mahendra K. Sunkara, Ph.D. 


\section{ACKNOWLEDGEMENTS}

I would like to thank my research advisor, Dr. Kyung A. Kang, for her guidance, education, and patience. I would also like to thank the committee members, Drs. Paula J. Bates, Michael H. Nantz, Mahendra K. Sunkara, and Thomas L. Starr, for their invaluable discussion and comments.

Dr. Achilefu and his group members, especially Dr. Sharon Bloch, from the Department of Radiology, Washington University, and Dr. Nantz and his group members from Chemistry Department, University of Louisville are recognized for their joint research effort. Dr. Tariq Malik and Ms. Lavona Casson in the Bates group in the James Graham Brown Cancer Center are acknowledged for their work and consultancy on cell study. Dr. Jacek Jasinski in the Conn Center for Renewable Energy Research at University of Louisville is acknowledged for consulting the theoretical study and helping with the TEM imaging. Dr. Robert Lupitskyy in the Kang group is acknowledged for help in the experiment. Many thanks are to the faculty and students of Chemical Engineering Department and Speed School of Engineering and my colleagues, who have given me helpful suggestions and assistance.

University of Louisville is acknowledged for the University fellowship that supported me for my first two years of study. The Institute for Molecular Diversity and Drug Design $\left(\mathrm{IMD}^{3}\right)$ is acknowledged for the graduate fellowship. School of Interdisciplinary and Graduate Studies at the University of Louisville is acknowledged for the Doctoral Dissertation Completion Award. 
U.S. Army (DOD) Breast Cancer Program (BC074387) is acknowledged for the financial supports for the research project. 


\begin{abstract}
THEORETICAL AND EXPERIMENTAL STUDIES ON MANIPULATION OF FLUORESCENCE BY GOLD NANOPARTICLE: APPLICATION FOR MOLECULAR IMAGING

Jianting Wang

December 2011
\end{abstract}

Gold nanoparticles (GNPs) have shown beneficial properties for biomedical use, e.g., their non-toxic nature and surface properties for easy modification. Upon receiving light, they generate a strong surface plasmon field, which can alter the fluorescence of fluorophores. The level and type of the fluorescence alteration depend on the GNP size and shape, excitation (Ex)/emission (Em) wavelengths and quantum yield of the fluorophore, as well as the distance between the fluorophore and GNP.

In this dissertation, the effect of the properties listed above on the fluorescence output was theoretically analyzed for the fluorophores frequently used in biomedical studies. For fluorescence quenching, fluorophores with the Em wavelength near the GNP plasmon resonance peak $(520 \mathrm{~nm})$ are better suited. As the Em wavelength increases, a shorter distance is required for achieving the same level of quenching. A bigger GNP requires shorter distance for quenching. To obtain fluorescence enhancement, the Em wavelength of the fluorophore needs to be longer than the GNP plasmon resonance peak (e.g., > $650 \mathrm{~nm}$ ). The fluorophore with lower intrinsic quantum yield tends to be 
enhanced more. The GNP needs to be sufficiently large ( $>5 \mathrm{~nm}$ ), and a bigger GNP provides a higher maximum enhancement.

Utilizing the quenching/enhancement ability of GNPs, a near-infrared (NIR) contrast agent that emits fluorescence at a higher level only at the particular cancer site was developed. Cypate, a safe NIR fluorophore, was selected as the fluorophore because NIR penetrates deeper into tissue and because Cypate is non-toxic. Cypate was conjugated to a GNP via two spacers. One is short for the quenching and with a substrate for a breast cancer-specific enzyme, urokinase-type plasminogen activator (uPA). The other is a long, biocompatible polymer chain for fluorescence enhancement. The fluorescence of the contrast agent was quenched by GNP by $93 \%$. In the presence of uPA, the short spacer was cleaved and the remaining long spacer enhanced fluorescence 1.8 times.

The study results are beneficial for developing efficacious optical contrast agents. This novel contrast agent can detect and diagnose breast cancer with high specificity and sensitivity, as FRET or molecular beacon but with a higher sensitivity and without the restriction of using DNA/RNA segments. 


\section{TABLE OF CONTENTS}

PAGE

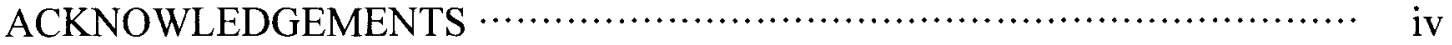

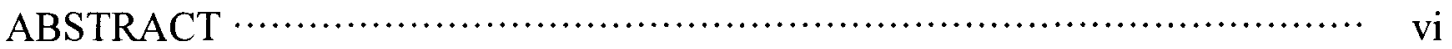

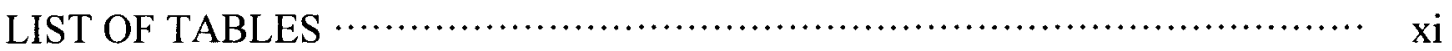

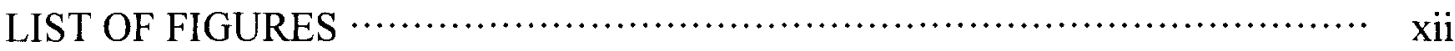

\section{CHAPTER}

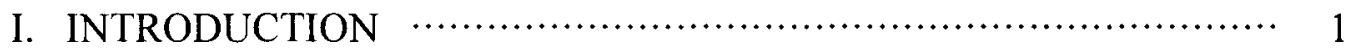

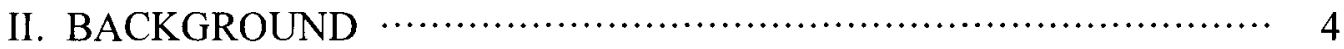

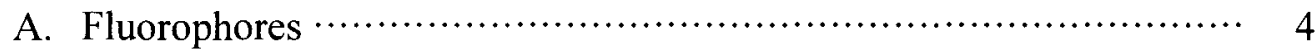

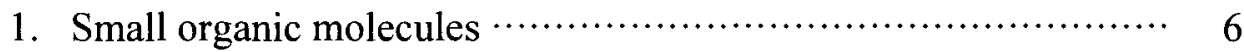

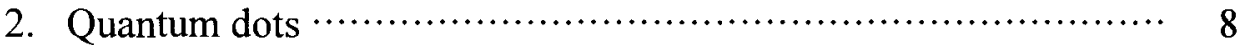

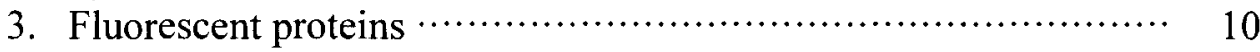

4. Specially designed fluorescent probes …........................ 11

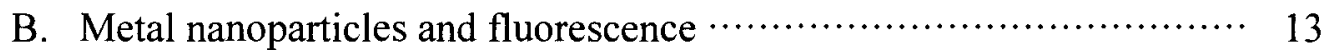

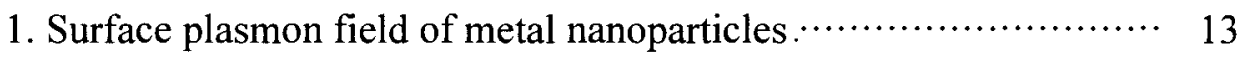

2. Fluorescence alteration by gold nanoparticles …................. 14

C. Theory of fluorescence alteration by metal nanoparticles $\cdots \ldots \ldots \ldots \ldots . .16$

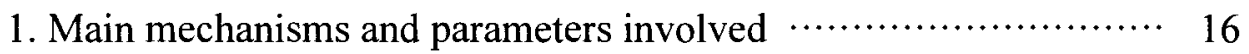

2. Mathematical model for plasmon field strength near a GNP ….... 18

(a) Plasmon field

(b) Fluorescence change of a fluorophore near a GNP

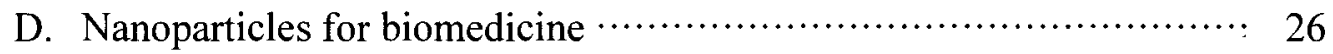

1. Advantages of nanoparticles for biomedical application $\cdots \ldots \ldots \ldots \ldots 26$ 
2. Types of nanoparticle

(a) Organic nanoparticles

(b) Inorganic nanoparticles

E. Fluorescence alteration by GNP studied by the Kang group …........... 34

III. MATERIALS, INSTRUMENTS, AND METHODS

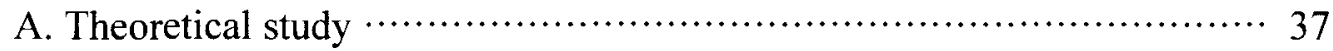

B. Syntheses of molecular spacers and modification of Cypate ……......... 38

1. Materials ……........................................................ 39

2. Instruments ………............................................... 39

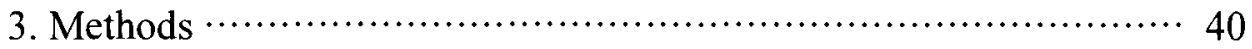

(a) Synthesis of the short spacers

(b) Conjugation of Cypate to short spacers

(c) Synthesis of long spacers

(d) Modification of Cypate

C. Conjugation of Cypate to GNPs

1. Materials …….................................................. 44

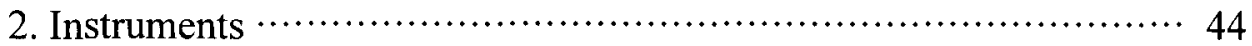

3. Methods …............................................................ 45

(a) Handling of thiolated materials

(b) GNP surface modification and Cypate conjugation

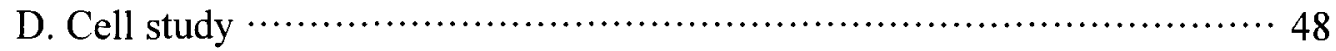

1. Materials ……........................................................... 48

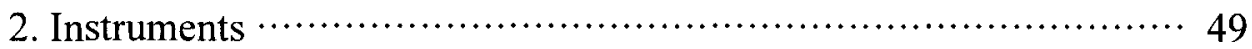

3. Methods ……............................................................ 49

(a) Quantification of uPA in cell media by ELISA

(b) Cell studies with GNP-SSP-Cy

(c) Cell studies with GNP-LSP-mCy

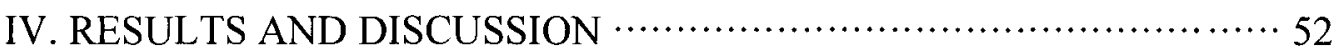

A. Theoretical Analysis of Fluorescence Alteration by GNP …….......... 53

1. Excitation Rate and System Parameters …………................... 53

(a) GNP size and incident light wavelength

(b) Effect of coating material on GNP 


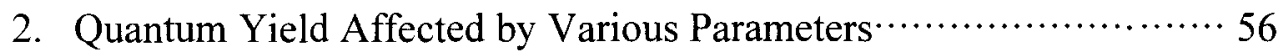

(a) Radiative decay rate

(b) Absorption

(c) Intrinsic quantum yield

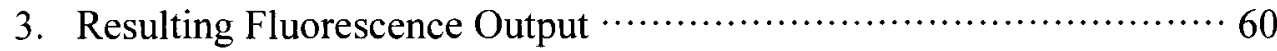

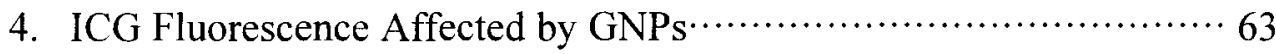

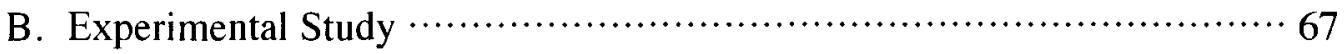

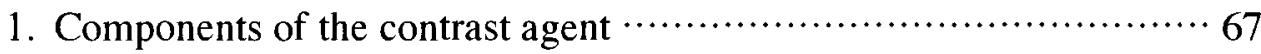
(a) GNP
(b) GNP surface modification
(c) Spacers
(d) Fluorophore
(e) Targeting molecule

2. Characterization of components

(a) The effect of the solvent on Cypate fluorescence

(b) Cypate concentration and self-quenching

(c) Fluorescence of free Cypate affected by free GNP colloids

3. Optimization of spacers

(a) Short spacer

(b) Long spacer

(c) Dual-spacer

4. Cell study

(a) Short spacer performance

(b) Long spacer performance

V. CONCLUSIONS

VI. FUTURE WORK

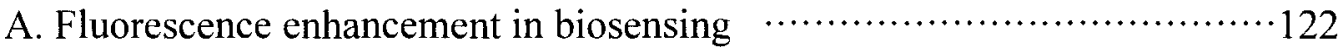

B. Hollow gold nanospheres for conditional fluorescence quenching $\cdots \cdots \cdots \cdot 133$ 


\section{LIST OF TABLES}

TABLE

PAGE

1. Fluorophores studied, and their excitation/emission peak wavelengths and intrinsic quantum yields.

2. Molecular spacers used for conjugating Cypate to GNPs.

3. Normalized radiative decay rate and absorption rate at $0.1,5$ and $10 \mathrm{~nm}$ from a $30 \mathrm{~nm}$ GNP surface, and $\left(1-q^{\circ}\right) / q^{\circ}$ of the fluorophores.

4. Spacers used and their estimated lengths by molecular simulation.

5. Size of nanoparticles, before and after conjugation of sPEG and sPEG/LSPs, measured by light scattering particle size analyzer. 


\section{LIST OF FIGURES}

FIGURE

PAGE

1. Mechanisms ( $a$ and $b$ ) and the parameters (1 4) involved in the fluorescence alteration by GNPs.

2. (a) The coordinates used in the computation of the plasmon field strength on and around a GNP and (b) a schematic diagram of a GNP with polymer coating in a medium.

3. GNP stabilized with (a) large poly ethylene glycol molecules and (b) with small aliphatic-PEG molecules.

4. The enhancement in the excitation decay rates of fluorophores by their respective Ex wavelengths, with respect to the distance from the surface of a

(a) $10 \mathrm{~nm}$ (b) $30 \mathrm{~nm}$ and (c) $50 \mathrm{~nm}$ GNP.

5. Plasmon field distributions computed on/around a $10 \mathrm{~nm}$ GNP coated with PEG, at a thickness of $0,1,2$, or $3 \mathrm{~nm}$, when the incident light at $780 \mathrm{~nm}$ is applied.

6. Absorption rate $\left(\gamma_{\text {dbs }} / \gamma_{t}^{\prime \prime}\right)$ of various fluorophores by a $30 \mathrm{~nm}$ GNP, with respect to the distance from the GNP surface.

7. Theoretically computed quantum yield of (a) various fluorophores

(b) ICG with respect to the distance from a $30 \mathrm{~nm}$ GNP.

8. Theoretical estimation of fluorescence output of various fluorophores affected by (a) 10 (b) 30 and (c) $50 \mathrm{~nm}$ GNP.

9. The enhancement in the excitation decay rates of ICG

(Ex wavelength $780 \mathrm{~nm}$ ) by GNPs at various sizes.

10. (a) Absorption rate $\left(\gamma_{a b s} / \gamma_{r}^{\prime \prime}\right)$ of ICG by a $30 \mathrm{~nm}$ GNP, with respect to the distance from the GNP surface (b) magnified view of the section in the rectangle in (a). 
11. Theoretically computed quantum yield of ICG (Ex/Em 780/830 nm) with the distance from the GNP at various sizes.

12. Theoretically computed fluorescence emission of ICG (Ex/Em 780/830 nm) with the distance from the GNP at various sizes.

13. (a) Design of highly cancer specific fluorescing cancer locator The agent normally does not emit fluorescence because the short spacer place Cypate very close to the GNP. (b) When the cancer locater binds to the cancer cell, the short spacer is cleaved by enzyme from cancer cell and the fluorescence is emitted at an enhanced level.

14. Molecular structures of (a) ICG and (b) Cypate (c) m-Cypate …............... 70

15. Fluorescence of Cypate in water, 1 and $10 \mathrm{mM}$ PBS buffer. …….............. 72

16. Fluorescence of Cypate at various Cypate concentrations. ……................. 73

17. Fluorescence of free Cypate with various concentrations of free GNP.

18. Schematic diagram for fluorescence of free Cypate affected by (a) high and (b) low GNP concentration in solution.

19. A schematic diagram of the uPA triggered fluorescence contrast agent, Cypate conjugated GNP via a short spacer: (a) normally emits little fluorescence (b) in the presence of uPA enzyme, the spacer is cleaved and the fluorescencing ability of the agent is restored.

20. (a) Absorption spectra of GNP, $s$ SP-Cy and GNP-sSP-Cy, and (b) TEM image of GNP-sSP-Cy [GNP, $8.0 \mathrm{~nm}$ ].

21. Relative fluorescence of the GNP-SSP-Cy with the core GNP size of 3.7, 8.0 and $16.4 \mathrm{~nm}$ at $10 \mu \mathrm{M}$ Cypate concentration in PBS buffer.

22. Theoretical estimation of fluorescence emission with the distance from the GNP surface for the GNPs sizes at 3.7, 8.0, and $16.4 \mathrm{~nm}$ for ICG $(\mathrm{Ex} / \mathrm{Em} 780 / 830 \mathrm{~nm})$.

23. The relative fluorescence levels of GNP-SSP-Cy with 3.7

(GNP concentration $31 \mathrm{nM}$ ) and $8.0 \mathrm{~nm}$ (GNP concentration $7 \mathrm{nM}$ ) GNPs at $10 \mu \mathrm{M}$ Cypate in PBS buffer, before and after adding uPA. Reaction time: $5 \mathrm{~min}$; Ex/Em: 780/830 nm.

24. Fluorescence of GNP- $n S S P-m C y$ with $8.0 \mathrm{~nm}$ GNP at various GNP concentrations, before and after adding uPA, Reaction time: $5 \mathrm{~min}$; $\mathrm{Ex} / \mathrm{Em}: 780 / 830 \mathrm{~nm}$. 
25. TEM image of $8.0 \mathrm{~nm}$ GNPs coated with (a) sPEG only and (b) sPEG and C12PEG6

26. Absorption spectra of $8.0 \mathrm{~nm}$ GNP, GNP coated with sPEG alone, with sPEG and C12PEG6, GNP-LSP-mCy, and mCy.

27. Schematic diagram of the function of blocking agent.

28. Relative fluorescence of $\mathrm{mCy}$ conjugated to $8.0 \mathrm{~nm}$ GNP via spacers with various lengths.

29. Relative fluorescence of mCy-BLK conjugated to $8.0 \mathrm{~nm}$ GNP via the spacers with various lengths, compared to the theoretically estimated fluorescence.

30. Relative fluorescence emjamcement of $\mathrm{mCy}$ conjugated to (a) 3.7 (b) 8.0 and (c) $16.4 \mathrm{~nm}$ GNPs via spacers with various lengths, compared to the theoretically estimated fluorescence level.

31. Original design for the synthesis of GNP-DSP-mCy (ideal case).

32. Potential undesired product (a) incomplete conjugation (b) cross linking from the original design for the synthesis of GNP-DSP-mCy. 94

33. The chemical structure of the ring-shaped dual spacer ( $r D S P)$.

34. Absorption spectra of $8.0 \mathrm{~nm}$ GNP, GNP coated sPEG, GNP-rDSP-mCy, and $\mathrm{mCy}$.

35. Fluorescence of GNP-rDSP-Cy (8.0 nm GNP) at $100 \mathrm{nM}$ Cypate, $0.9 \mathrm{nM}$ GNP, before and after adding uPA at 1030 units $/ \mathrm{ml}$ $(\mathrm{Ex} / \mathrm{Em} 780 / 830 \mathrm{~nm})$.

36. uPA concentrations in the media containing MCF-7 (uPA-) or MDA-MB-231 (uPA + ) cells, after 8 and 32 hrs of cell culturing .

37. Fluorescence of GNP-SSP-Cy in media without cells, with MDA-MB-231 cells, and with MCF-7 cells after $2 \mathrm{hr}$ incubation. Ex/Em: 780/805-830 nm. 100

38. Fluorescence of Cypate and GNP-LSP-mCy in (a) MCF-7 (uPA-) and (b) MDA-MB-231 (uPA+) cells, 1, 3 and $24 \mathrm{hrs}$ after the treatment. $\cdots \cdots \cdot 101$

39. Fluorescence of Cypate and GNP-LSP-mCy in (a) MCF-7 (uPA-) and (b) MDA-MB-231 (uPA+) cells, 1, 3 and $24 \mathrm{hrs}$ after the treatment. 


\section{CHAPTER I \\ INTRODUCTION}

Fluorophores have been extensively used as a signal mediator in the biomedical research and disease diagnosis/prognosis. Artificial manipulation of the fluorescence of a fluorophore can be highly beneficial for improving the specificity and/or sensitivity of biosensing/bioimaging. For example, fluorescence quenching can be used for negative sensing or for conditional de-quenching to increase specificity. Fluorescence enhancing can improve sensitivity and the signal to noise ratio.

Since fluorescence is generated by the electrons that are excited by photonic energy, a localized entity affecting the electron state of the fluorophore can change the fluorescence output. Nano-sized metal particles forming a strong electromagnetic (plasmon) field upon receiving photonic energy can be, therefore, good candidates for this purpose [Lakowicz, 2005; Zayats, et al., 2005; Evanoff and Chumanov, 2005]. Gold nanoparticles (GNPs) are one of the most attractive metal nanoparticles for biomedical applications for this purpose because they are chemically stable and non-toxic and also because the gold-thiol chemistry for surface conjugation of biomolecules is well established [Connor, et al., 2005; Love, et al., 2005; Shukla, et al., 2005].

Fluorescence quenching and enhancement using GNPs have been explored by many researchers. The type and the level of fluorescence alteration by GNPs depend on parameters including the metal type, particle size; the excitation and emission 
wavelengths and the quantum yield of the fluorophores; and the distance between the fluorophore and the particle. In this dissertation, the effect of these parameters on the plasmon field distribution of GNPs and on the fluorescence alteration was analyzed for commonly used fluorophores in biomedical studies. The study result is expected to provide information for selecting fluorophore, GNP size, distance between the fluorophore and the GNP, in designing the efficacious contrast agents for molecular imaging.

In parallel to the theoretical analyses, a safe, near-infrared (NIR) contrast agent for cancer detection was developed. The contrast agent specifically targets the cancer cell, and it emits fluorescence, only in the presence of the enzyme secreted by a particular breast cancer type, at a highly enhanced level. Thus, it provides high specificity and sensitivity for cancer detection. It can also be used to characterize the cancer type (diagnosis) by its ability of recognizing the particular enzyme. The same approach can also be used for other disease diagnoses. This contrast agent can be potentially combined with cancer treatment function (e.g., photo-dynamic therapy, hyperthermia) to enable seamless diagnosis and treatment.

The structure of this dissertation after this introduction is as follows:

Chapter II presents the BACKGROUND information on the fluorophore, metal (especially gold) nanoparticles, fluorescence quenching and enhancement by GNPs, and the mechanism and mathematic models of fluorescence alteration by GNPs.

Chapter III describes the MATERIALS, INSTRUMENTS, AND METHODS used in theoretical and experimental studies. 
Chapter IV contains the RESULTS AND DISCUSSIONS of the theoretical analyses of the factors affecting fluorescence alteration by GNP, and the experimental study of fluorescence quenching, restoration, and fluorescence enhancement of Cypate by GNPs.

Chapter V concludes (CONCLUSIONS) the studies performed.

In Chapter VI, suggested FUTURE WORKs are presented.

In the APPENDIX, the application of gold nanoparticle reagent in our fiber-optic biosensing system, and fluorescence alteration of Cypate by hollow gold nanosphere are presented. 


\section{CHAPTER II}

\section{BACKGROUND}

\section{A. FLUOROPHORES}

Fluorophores are molecules that absorb light at a particular wavelength and emit light at another but equally specific wavelength [Hercules, 1966]. The process is such that the electrons of the fluorophore absorb photons and the photons raise the energy level of the electrons to an excited state. During the excitation period, some of the energy is dissipated by molecular collisions or transferred to a proximal molecule, and then the remaining energy is emitted as photons to relax the electrons back to their ground state. Because the emitted photons usually carry energy less than the one received, they are emitted at a longer wavelength. The wavelength shift between the excitation and emission lights is called the Stokes Shift and each fluorophore has its distinct characteristic Stokes shift. If the Stokes Shift is very small and the excitation and emission wavelengths greatly overlap, the detection of emitted fluorescence can be difficult to distinguish from the excitation light. Conversely, fluorophores with large Stokes shifts are easier to use because the two can be distinguished better [Lakowicz, 2006]. Since the emitted light (fluorescence) can be distinguished from the excitation light and is at a particular wavelength, one can use it as an optical signal [Hercules, 1966; Rendell, 1987; Guilbault, 1990]. Fluorophores have been, therefore, extensively used in 
biosensing and imaging for detecting biomolecules, monitoring/studying biological processes, diagnosing/prognosing diseases, etc. [Choy, et al., 2003].

Early studies using fluorophores employed those emitting the light mainly in the visible range of the electromagnetic spectra (e.g., 400-700nm) because of the limitation in detection. The advancement in technology has allowed the fluorescence detection beyond the visible spectra and into the ultraviolet (UV, e.g., 10-400 nm) and near infrared (NIR) ranges (e.g., 700-900 nm). The new fluorophores and detectors offer greater variability, versatility, and multiplexing capabilities.

The quantum yield (QY) of a fluorophore is the ratio of the number of photons emitted as fluorescence to that of the photons absorbed, i.e., the emission efficiency of the fluorophore [Lakowicz, 2006]. QY is unique for each fluorophore. In sensing or imaging applications, fluorophores with higher QY is desired because they provide greater sensitivity.

Photostability is another important property of a fluorophore. When a fluorophore is exposed to an excitation light, the fluorescence level decreases over time due to photon-induced chemical damage, and the decrease in the fluorescence is called photobleaching [Lakowicz, 2006]. Photostability is very important for optical imaging, especially in optical microscopy where the excitation light intensity is high.

Other important properties of fluorophores may include functional groups for bioconjugation, water-solubility, toxicity, pH-sensitivity, etc. Depending on the application, the fluorophore with desired properties needs to be carefully selected. 


\section{Small Fluorescent Organic Molecules}

Small fluorescent organic-molecules are often called organic dyes. Organic dyes are small molecules, at the scale of $\sim 0.5 \mathrm{~nm}$ [Resch-Genger, et al., 2008]. The small size of these fluorophores is an advantage for bioapplications because they can be conjugated to macromolecules, such as antibodies, biotin or avidin, without interfering with their biological function. The first fluorescent compound used in biological research was Fluorescein synthesized by Adolf von Baeyer in 1871 [Guilbault, 1990; Resch-Genger, et al., 2008; Zhang, et al., 2002; Waggoner, 2006]. Fluorescein is still widely used today and its derivatives have been produced to improve their photostability and solubility. Currently a wide variety of dyes are available with various excitation/emission spectra, optimal quantum yields, excitation coefficients and functional groups for conjugation are available. Examples include rhodamine (TRITC), coumarin, cyanine, Alex Fluors, and their derivatives [Rietdorf, 2005].

NIR fluorophores have attracted much attention especially in optical imaging of human because they offer several advantages over the fluorophores in the visible range. NIR is absorbed less by biological tissues than the visible light. In tissue, there is less autofluorescence in NIR than in the visible range, thereby less background noises [Kobayashi, et al., 2002]. In the infrared region, photons are absorbed by water much more than in NIR. NIR, therefore, provides a window of opportunity with deeper tissue penetration, i.e., 2-4 centimeters [Marshall, et al., 2010]. Compared to other commonly used imaging techniques, such as magnetic resonance imaging (MRI), computed tomography (CT), and photon emission tomography (PET), optical imaging is more 
sensitive, more cost-effective, rapid, and easy to use [Choy, et al., 2003; Cassidy and Radda, 2005; Mankoff, 2008, Marshall, et al., 2010].

The early NIR imaging usually used angiogenesis-mediated absorption contrast. The absorption of NIR light in a typical tissue having $8 \%$ blood volume and $29 \%$ lipid content, the dominant absorber is hemoglobin, accounting for 39-64\% of total absorbance at NIR wavelength [Lim, et al., 2003]. NIR optical tomography using time-dependent measurements of light propagation in the breast has been developed for breast cancer screening for two decades [Kang, et al., 1993; Ntziachriostos and Chance, 2001; Honar and Kang, 2002; Wang, et al., 2010]. There has been remarkable advance in the application of NIR on detecting the angiogenesis and increased hemoglobin absorbance for contrast. However, the angiogenesis-mediated absorption contrast only for optical mammography reduces the potential for using NIR techniques to assess sentinel lymph node staging, metastatic spread, and multifocality of breast disease [Frangioni, 2003; Hawrysz and Sevick-Muraca, 2000]. Recent research has been focused on the use of contrast agents for the molecular-based, diagnostic imaging [Marshall, et al., 2010].

Exogenous, optical contrast agents for human use have been limited to the safe organic dyes. In 1959, Indocyanine green (ICG) was approved by the United States Food and Drug Administration (FDA) as a contrast agent for retinal angiography for both absorption and fluorescence imaging. Currently, it still remains the only FDA-approved NIR fluorophore [Alford, et al., 2009; Marshall, et al., 2010]. Fluorescent imaging with ICG has been used for the vascular mapping and tissue perfusion measurement [Frangioni, 2003]. ICG angiography is a routine test in the ophthalmology clinic for detecting the posterior uveitis [Herbort, et al., 1998] and central serous chorioretinophthy 
[Chen, et al., 1999]. ICG has been also used to image the vasculature [Raabe, et al., 2003] and cerebrospinal fluid in brains [Sakatani, et al., 1997] of both rats and human, for improved tumor localization and the assessment of post-resection margins with high sensitivity and specificity [Haglund, et al., 1994]. It has also been studied for sentinel lymph node mapping for cancer staging in breast, skin and gastric cancers [Marshall, 2010].

\section{Quantum dots}

Quantum dots (QDs) are semiconductor nanocrystals and fluorescent nanoparticles $(2-10 \mathrm{~nm})$ that contain a core of the group II and VI elements or group III and $\mathrm{V}$ elements [Gao, 2005; Medintz, 2005]. $\mathrm{CuCl}$ nanocrystals in colloidal solutions are first discovered in the 1980s by Alexei Ekimov [1981] and by Rossetti [1983], and the term "quantum dot" was first used by Mark Reed [1988]. In 1993, Murrays, et al. [1993] developed the procedure for synthesizing of high-quality CdSe QDs dispersed in organic solvents.

In QDs, the excitons are confined in all three spatial dimensions due to the small size of the particle. Therefore, the electronic characteristics of these particles are closely related to the size and shape of the individual crystal. Generally, smaller QD results in larger band gap, which means the difference in energy between the highest valence band and the lowest conduction band is greater. Therefore, more energy is needed to excite the QD, and thus, more energy is released when the crystal returns to its resting state. As a result, by changing the size and shape of the particle, the excitation and emission 
wavelengths can be tuned, typically from the visible to NIR range [Reed, et al., 1988; Wang, et al., 2001; Michalet, et al., 2001; Altinoglu and Adair, 2010].

Compared to organic dyes, QDs have higher quantum yield [ $>50 \%$, West and Halas, 2003] and higher molar extinction coefficients, e.g., roughly an order of magnitude higher than even the strongly absorbing Rhodamine 6G [Leatherdale, et al., 2002; Smithpeter, et al., 1998]. As a result, they emit brighter fluorescence (most QDs are approximately 10-20 times brighter) than organic dyes [Dabbousi, et al., 1997; Chan and Nie, 1998]. Since the light absorption by QDs is greater at a shorter wavelength (higher energy), QDs exhibit an almost continuous, broad-band absorption and a narrow emission spectrum, resulting in a large effective Stokes shifts [Cai, et al., 2007; Michalet, et al., 2005]. Therefore, the excitation and emission spectra of QDs are well separated. QDs also exhibit photostability higher than organic dyes [Santra and Dutta, 2007].

QDs became fluorescent probes for the biosystem in 1998 when two groups simultaneously reported the procedures for making water-soluble QDs and conjugating them to biomolecules [Chan and Nie, 1998; Bruchez et al., 1998, Mazumder, et al., 2009]. Since then, extensive research has been directed toward using QDs in biosensing and bioimaging. QD labeled microorganisms, biomarkers, cancer cells, etc. have been studied in detecting biomolecules and in disease diagnosis, but not in humans [Mazumder, et al., 2009]. Even though NIR QDs have a great potential, there are only a few reports for in vivo NIR imaging using QDs [Altinoglu and Adair, 2010], and all of these reports employed QDs as a tracer in sentinel lymph node mapping. Although some researchers assert that the surface modification of QDs may eliminate the potential harm [Gerion, et al., 2001; Altinoglu and Adair, 2010; Resch-Genger, et al., 2008], the 
cytotoxicity to organelles and membranes, induced apoptosis, and peroxidative stress, are still serious concerns in using QDs for human [Jaiswal and Simon, 2004; Hoshino, et al., 2004; Ballou, et al., 2004; Ryman-Rasmussen, et al., 2007].

\section{Fluorescent proteins}

Green fluorescent protein (GFP) was originally isolated from the light-emitting organ of the jellyfish Aequorea victoria by Shimomura, et al., in 1962. Thirty years later, Chalfie, et al. [1994] characterized the DNA encoding of GFP and used it as a gene expression reporter. In 2006, Martin Chalfie, Osamu Shimomura, and Roger Y. Tsien shared a Nobel Prize in Chemistry for their discovery of GFP. Since then, derivatives of the GFP and many other fluorescing proteins were developed in biological expression systems, and they are now frequently used in biological research [Choy, et al., 2003]. The benefit of using this type of fluorophores is that the plasmid expression can be introduced into cells, organs or organisms, to express the fluorophore either alone or fused to the protein of interest in the biological processes studied [Miyawaki, et al., 2003]. However, the use of fluorescent proteins can be time consuming, and also expressing large amounts of light-producing proteins can cause reactive oxygen species, introducing artifactual responses or toxicity. In addition, the size of the fluorescent protein may change the biological function of the cellular protein to which the fluorophore is fused. Biological fluorophores do not typically provide the level of photostability and sensitivity offered by synthetic fluorescent dyes [Prasher, et al., 1992; Kain, et al., 1995; Choy, et al., 2003].

In terms of the usage of fluorophores for human, until now, only a few organic dyes are used for human due to the safety issue [Kovar, et al., 2007]. Although several 
organic dyes are used for in vivo animal studies, for humans, only fluorescein and ICG were approved by the US FDA [Alford, et al., 2009], and for NIR, ICG is the only one. Cypate, a derivative of ICG, is demonstrated to be non-toxic to animals up to 10 $\mu \mathrm{mol} / \mathrm{kg}$, and considered to be a "biocompatible" dye [Achilefu, et al., 2002], although it has not yet been submitted for FDA approval.

\section{Specially designed fluorescent probes}

The fluorescence emission level can be influenced by interactions with other fluorescing or non-fluorescing molecules. These interactions can be beneficially used to design probes highly specific to a particular biomolecule for biosensing or molecular imaging, in such a way that the fluorescence level or emission wavelength changes in the presence of the biomolecule. The most frequently used fluorescent probes include the molecular beacon and the fluorescence resonance energy transfer (FRET, also known as Förster resonance energy transfer).

Molecular beacon was first developed by Tyagi and Kramer [1996]. In the molecular beacon. a fluorophore and a quencher are attached to the two ends of a hairpinshaped oligonucleotide. When a fluorophore is in contact with a quencher, the energy from excitation is transferred to the quencher, and this energy is then lost by heat [O'Reilly, 1975]. In the absence of a complimentary target sequence, the beacon remains closed and there is no appreciable fluorescence. Once the single stranded loop portion of the hairpin hydridizes to the target sequence. the resulting spatial separation of the fluorophore from the quencher leads to fluorescence emission. Molecular beacons have become widely used in chemistry, biology, biotechnology and medical sciences for the 
particular biomolecular recognition, due to the easy synthesis, molecular specificity and structural tolerance to various modifications [Tan, et al., 2004]. For example, molecular beacons have been commonly used for real-time monitoring of DNA or RNA amplification during polymerase chain reaction (PCR) [Li and Tan, 2003]. Since the initial development there have been various modifications of molecular-beacon sensors, including miniaturized surface-immobilized hybridization assays and microarrays [Vet, $e t$ al., 2002; Broude, 2002; Lou and Tan, 2002, Epstein, et al., 2003]. Molecular beacons have also been used in monitoring genes in living cells.

FRET occurs between two fluorophores. Excitation energy is transferred from a donor to an acceptor fluorophore. In the donor-acceptor pair, the emission wavelength of the donor fluorophore must match the excitation wavelength of the acceptor molecule [Didenko, 2001]. As a result, the donor molecule fluorescence is quenched, and the acceptor molecule becomes excited. It then loses energy via heat or the fluorescent emission [Didenko, 2001]. The initial theory of FRET was developed by Theodor Förster in 1948. The distance dependent FRET was experimentally confirmed by Stryer and Haugland [1967] and Haugland, et al. [1969]. For FRET to occur, the distance between the donor and the acceptor molecule must be within a distance of $0.5-10 \mathrm{~nm}$, depending on the specific donor-acceptor pair. The first biological applications of this phenomenon started in the 1970s, dealing primarily with protein research [Didenko, 2001]. FRETbased DNA applications began to appear in the mid-1980s and 1990s and has gained popularity because of the availability of the fluorescent protein [Piston, 2007; Miyawaki, et al., 1997; Patterson, et al., 2001; Chalfie, et al., 1994]. The detection of FRET and its disruption are both used in the assays. In FRET probes, the fluorophores are usually 
labeled onto two oligonucleotides separately, and when these two oligonucleotides form a duplex, bringing the two fluorophores in close proximity, FRET occurs. Alternatively, the hairpin configuration of the oligonucleotide labeled with the donor and acceptor fluorophores can also result in FRET. DNA-based FRET probes are used in vitro and in vivo in many applications monitoring various types of DNA and RNA reactions, including PCR, hybridization, ligation, cleavage, recombination, and synthesis. They are also applied in sequencing, mutation detection, and as parts of biosensors to assess the concentration of lead, DNA/RNA and protein [Didenko, 2001; Piston, 2007].

\section{B. METAL NANOPARTICLES AND FLUORESCENCE}

With the advance in nanotechnology, the fluorescence alteration by metal nanostructures has attracted much interest. These nanostructures are able to not only quench the fluorescence, but also enhance the fluorescence intensity, providing the sensing/imaging with the potential to increase the sensitivity and specificity.

\section{Surface plasmon field of metal nanoparticles}

Surface plasmon is coherent electron oscillation that exists at the metal-dielectric material interface, such as a thin metal film, a small metal particle, or a sharp metal tip. The existence of surface plasmon was first predicted in 1957 by Ritchie [1957]. The localized surface plasmon polariton is collective electron-charge-oscillation in metallic nanoentities, including nanoparticles, excited by light. The surface plasmon polariton can propagate along the metal surface until the energy is lost either by the absorption by the metal or by the radiation into the free-space. This propagating energy forms a surface plasmon field near the metal surface. For metal nanoparticles, the surface plasmon is 
highly localized near the surface and rapidly decays from the particle surface into the dielectric environment [González-Díaz, et al., 2008]. The local surface plasmon exhibits enhanced field strength at around the resonance wavelength and it is the highest at the resonance wavelength. The resonance condition is determined by the absorption spectroscopy and depends on the size and shape of the particle and dielectric properties of both the metal and the surrounding material. For noble metals, such as gold and silver, the resonance wavelength is in the visible light range [Evanoff and Chumanov, 2005; Kreibig and Vollmer, 1995]. Therefore, gold and silver nanoparticles have been studied extensively in their interaction with optical probes, such as fluorophores [Lakowicz, 2005; Zayats, et al., 2005].

For bio-application, gold nanoparticles (GNPs) have been studied the most because they have been well characterized, and they have little to no long-term toxicity or other adverse effect in vivo [Connor, et al., 2005; Shukla, et al., 2005]. Colloidal gold has been safely used to treat rheumatoid arthritis for over 50 years [Merchant, 1998; Root, et al., 1954]. Recent study results indicate that the colloidal GNPs coated with a protective layer of polyethylene glycol (PEG) exhibit excellent in vivo biodistribution and pharmacokinetic properties after systemic application [James, et al., 2007; Paciotti, et al.,

2004; Paciotti, et al., 2006]. Currently, a PEG coated GNP with targeting molecule is already in a phase I clinical trial for advanced-staged cancer patients [Libutti. et al., $2010]$.

\section{Fluorescence alteration by gold nanoparticles}

Since fluorescence is generated by the electrons excited by photonic energy, a localized entity affecting the electron state of the fluorophore can change the fluorescence 
output. GNP forming surface plasmon upon receiving photonic energy can be, therefore, good candidates for this purpose [Lakowicz, 2005; Zayats, et al., 2005]. When a fluorophore is placed very close to a GNP (e.g., within a few nanometers), the fluorescence is usually quenched because the fluorophore donates the excited electrons to the GNP, thus the electrons take the non-radiative pathway. However, as the distance increases the electric field (plasmon field) near the GNP is still strong but the GNP is not able to interact directly with the electrons of the fluorophore. Thus, the fluorescence can be enhanced [Eustis and El-Sayed, 2006].

The fluorescence quenching using GNPs has been studied by many researchers: Ghosh, et al. [2004] studied the effect of the GNP size on the fluorescence quenching; Schneider, et al. [2006] showed the degree of quenching as a function of the distance between a GNP and a fluorophore; Dulkeith, et al. [2002] observed a decrease in the radiative decay of a fluorophore placed near a GNP; Kato and Caruso [2005] utilized the quenching phenomenon in a competitive fluorescence immunoassay for biosensing and imaging; Dubertret, et al., [2001] used GNPs as fluorescence quenchers in DNA molecular beacons; etc.

Utilizing GNPs for enhancing the fluorescence of fluorophores has also been previously explored by several researchers. Nakamura et al. [2005] studied the level of the fluorescence enhancement with respect to the GNP size, for the fluorophore Rose Bengal; Kuhn, et al. [2006] and Anger, et al. [2006] observed the change in the fluorescence of Nile Blue with the distance from a GNP; Laurent and Asahi [2009] obtained fluorescence enhancement of a film of $N, N^{\prime}$-bis[2,5-di-tert-butylphenyl]3,4,9,10-perylene dicarboximide by a single GNP. 


\section{THEORY OF FLUORESCENCE ALTERATION BY METAL PARTICLES}

Although numerous studies have demonstrated the ability of fluorescence alteration by GNPs, to beneficially utilize fluorescence manipulation in designing contrast agents, it is very important to thoroughly understand the correlation between the manipulable parameters of the system and the fluorescence output. Theoretical analysis using the mathematical model may be one of the most efficient ways for this purpose.

\section{Mechanisms and Parameters}

The mechanism of fluorescence alteration by metal nanoparticles has been studied by many physicists as early as in 1980s. Gersten and Nitzan [1981], and Nitzan and Brus [1981] studied the fluorescence from a single dye molecule adsorbed on a spherical metal particle. They pointed out that the fluorescence change is a result of the competition among the enhanced excitation, enhanced radiative and nonradiative decay rates near the metal particle surface. They concluded that, for the dyes with low quantum yield, the fluorescence is suppressed directly on the metal particle surface, but increased to a maximum at a well-defined distance from the surface. These early theoretical speculations were later verified by experiments with silver islands deposited on silicon oxides [Wokaun, et al., 1983; Lakowics, 2005]. Anger, et al. [2006] and Kuhn, et al. [2006] verified this theoretical prediction of fluorescence using a single-GNP and singledye system. This mechanism has been well accepted by many researchers [Wokaun, $e t$ al., 1983; Anger, et al., 2006; Farahani, et al., 2005; Bharadwaj and Novotny, 2007], although these researchers may use different mathematical models for the fluorescence alteration by metal nanoparticles. 
The main mechanism of fluorescence alteration and the parameters affecting the fluorescence can be described as follows [Gersten and Nitzan, 1981; Bharadwaj and Novotny, 2007]: When a fluorophore is placed in an electromagnetic field (plasmon field) generated by a metal nanoparticle exposed to the incident light at a particular wavelength, two main changes occur with the fluorescence output: (a) its excitation decay rate (Fig. 1a) increases; (b) its the quantum yield (Fig. 1b) changes by the increase in the radiative molecular decay rate, by the re-routing of the radiative decay to nonradiative decay, and by the intrinsic non-radiative decay rate (intrinsic quantum yield) of the fluorophore.

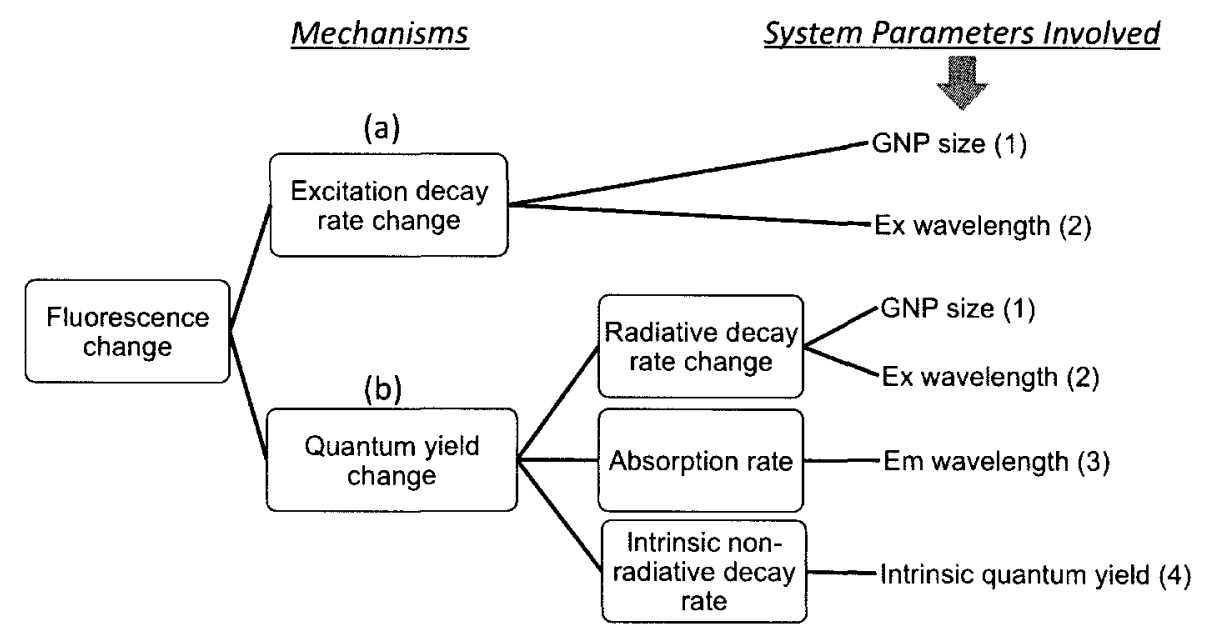

Figure 1. Mechanisms ( $a$ and $b$ ) and the parameters (1 4) involved in the fluorescence alteration by GNPs

The increase in the excitation decay rate is caused by the plasmon field generated by the GNP upon receiving the photonic energy. The level of the increase is determined by the plamson field strength, which depends on the GNP size and the wavelength of the incident light (in a practical sense, the excitation wavelength of the fluorophore) and the distance between the fluorophore and the GNP. The change in the quantum yield is 
affected by three different factors. (1) The increase in the radiative decay rate of a fluorophore is caused by the plasmon field [Gersten and Nitzan, 1981; Bharadwaj and Novotny, 2007]. (2) The non-radiative decay rate is induced by the GNP and it strongly depends on the emission (Em) wavelength of the fluorophore. (3) The intrinsic quantum yield of the fluorophore in the medium of interest also affects the results, especially when the value is exceptionally small [Gersten and Nitzan, 1981; Nitzan and Brus, 1981; Wokaun, et al., 1983].

The four main system parameters affecting the fluorescence change by a GNP are, therefore, the GNP size, the Ex and Em wavelengths, and the intrinsic quantum yield of the fluorophore (Fig. 1). In addition to these four, an important, manipulable parameter is the distance between a fluorophore and a GNP, because the plasmon field strength decays with the distance from the GNP. To achieve a desired fluorescence alteration by GNPs, therefore, it is helpful to thoroughly understand how these five parameters affect the fluorescence output.

\section{Mathematical model for plasmon field strength near a GNP}

\section{(a) Plasmon field}

The plasmon field strength around a metal nanoparticle has been frequently analyzed by the Mie theory [Born and Wolf, 1999; Hartling, et al., 2007]. The Mie theory is based on the scattering of electromagnetic radiation by spherical particles and it is an analytical solution of the Maxwell equation [Mie, 1908]. In biomedical applications, metal nanoparticles are usually coated with a polymer layer to stabilize the particle and increase the hydrophicility. Therefore, the effect of the layer on the field distribution 
must be considered. Neeves and Birnboim [1989] extended the previous model for bare metal nanoparticle to the one for the field strength near a core-shell nanoparticles dispersed in a dielectric medium. This particular model is limited to the dipolar contribution. The particle concentration is to be sufficiently dilute so that the interparticle interaction may be ignored, and the intrinsic dielectric non-linearity is neglected [Neeves and Birnboim, 1989]. The model uses spherical coordinate system (Fig. 2). The GNP (radius, $\mathrm{r}_{1}$ ) is placed in the center of the coordinate (Fig. 2a) and it is coated with a shell (thickness, $r_{2}-r_{1}$ ). The dielectric permittivities of the core, the shell and the medium are $\varepsilon_{l}, \varepsilon_{2}$, and $\varepsilon_{3}$, respectively (Fig. 2b). An incident light $\left(E^{\circ}\right)$ is applied in the $z$ direction, at $\theta=0$. The azimuthal symmetry $(d E / d \phi=0)$ is assumed.

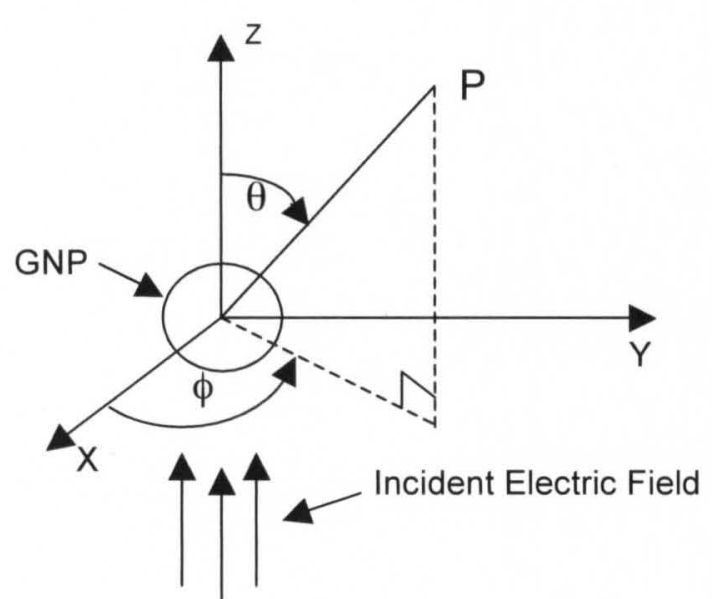

(a)

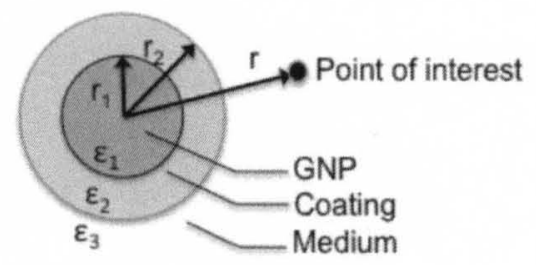

(b)

Figure 2. (a) The coordinates used in the computation of the plasmon field strength on and around a GNP and (b) a schematic diagram of a GNP with polymer coating in a medium.

The plasmon field strength $(E)$ at a position $r$ in the medium can be then described as in $\mathrm{Eq}(1)$. 


$$
\frac{E}{E^{o}}=\left[2 \frac{\varepsilon_{2} \varepsilon_{a}-2 \varepsilon_{3} \varepsilon_{b}}{\varepsilon_{2} \varepsilon_{a}+2 \varepsilon_{3} \varepsilon_{b}}\left(r_{1} / r\right)^{3}+1\right] \cos (\theta) \hat{e}_{r}+\left[\frac{\varepsilon_{2} \varepsilon_{a}-\varepsilon_{3} \varepsilon_{b}}{\varepsilon_{2} \varepsilon_{a}+2 \varepsilon_{3} \varepsilon_{b}}\left(r_{2} / r\right)^{3}-1\right] \sin (\theta) \hat{e}_{\theta},
$$

where $\hat{e}_{r}$ and $\hat{e}_{\theta}$ are the unit vectors in $r$ and $\theta$, respectively, and

$$
\begin{aligned}
& \varepsilon_{a}=\varepsilon_{1}(3-2 P)+2 \varepsilon_{2} P \\
& \varepsilon_{b}=\varepsilon_{1} P+\varepsilon_{2}(3-P) \\
& P=1-\left(r_{1} / r_{2}\right)^{3}
\end{aligned}
$$

The factor $P$ is the ratio of a shell volume to total particle volume. The field strength inside the shell ( $E^{\text {layer }}$; in our case, biopolymer coating) is:

$$
\begin{gathered}
\frac{E^{\text {layer }}}{E^{o}}=\frac{3 \varepsilon_{3}\left[\left(\varepsilon_{1}+2 \varepsilon_{2}\right)+2\left(\varepsilon_{1}-\varepsilon_{2}\right)\left(r_{1} / r\right)^{3}\right]}{\varepsilon_{2} \varepsilon_{a}+2 \varepsilon_{3} \varepsilon_{b}} \cos (\theta) \hat{e}_{r} \\
-\frac{3 \varepsilon_{3}\left[\left(\varepsilon_{1}+2 \varepsilon_{2}\right)-2\left(\varepsilon_{1}-\varepsilon_{2}\right)\left(r_{1} / r\right)^{3}\right]}{\varepsilon_{2} \varepsilon_{a}+2 \varepsilon_{3} \varepsilon_{b}} \sin (\theta) \hat{e}_{\theta} .
\end{gathered}
$$

When there is no shell on the GNP, $r_{l}=r_{2}, P=0$, and $\varepsilon_{I}=\varepsilon_{2}$, and hence, $\varepsilon_{a}=\varepsilon_{b}=3 \varepsilon_{l}$.

For a GNP, $\varepsilon_{l}$ is wavelength dependent. It is available as experimental data [Johnson and Christy, 1972], or it may be obtained by the Drude-Lorentz model (Eq. 6):

$$
\varepsilon_{1}(\omega)=\varepsilon_{o}\left(1-\omega_{p}^{2} \frac{1}{\omega^{2}+i \omega \gamma_{f}}+\omega_{p}^{2} \frac{1}{\omega_{o}^{2}-\omega^{2}+i \omega \gamma_{b}}\right)
$$

where $i$ denotes imaginary number; $\omega$ is the frequency of the incident light; $\omega_{0}$, bound electron resonant frequency; $\omega_{p}$, plasma frequency; and

$$
\gamma_{f}=1 / \tau_{f}=1 / \tau_{o}+V_{f} / r_{1} \text {, and } \gamma_{b}=1 / \tau_{b}
$$

$\tau_{f}$ and $\tau_{b}$ are the free electron scattering and the bound electron decay time, respectively. $V_{f}$ is the Fermi velocity and $\tau_{0}$, the free electron scattering time in the bulk material. The 
dielectric function of a GNP by Drude-Lorentz model has been confirmed to agree with the experimental data by Neeves and Birnboim [1989].

\section{(b) Fluorescence change of a fluorophore near a GNP}

The two approaches in developing the theoretical model for the fluorescence of a fluorophore affected by a metal nanoparticle are the steady state approach and the quantum mechanical approach. Gersten and Nitzan [1981] developed the models in both way and their models were often used by others.

\section{(b-i) Steady state model}

This model is for a system with a single fluorophore and a single metal nanoparticle. The relationship between an excited fluorophore and a metallic particle is presented with the dipolar and higher order multipolar contributions. For the particles with the size corresponding to the Rayleigh limit (dimensions $\leq \lambda / 10, \lambda$ is the wavelength of the incident light or fluorescence), only the dipolar part is relevant for fluorescence radiation. Gersten and Nitzan pointed out that the fluorescence change $(Y)$ of a fluorophore near a metal nanostructure is a product of two factors: $L\left(\omega_{\text {exc }}\right)$, the amplification number of the local field, and $Z\left(\omega_{f l u}\right)$, the partitioning of the excitation energy into the various radiative and non-radiative decay channels, by the presence of the particle.

$$
Y=\left|L\left(\omega_{e x c}\right)\right|^{2} Z\left(\omega_{f l u}\right)
$$

where $\omega_{e x c}$ is the excitation frequency. $L\left(\omega_{e x c}\right)$ can also be expressed as $E / E^{o}$ as used in equation (1). 
Equation (8) was used later by many other researchers [Kummerlen, et al., 1993; Nakamura, et al., 2005; Kuhn, et al., 2006; Bharadwaj and Novotny, 2007; Hartling, et al., 2007]. Bharadwaj and Novotny [2007] expressed this model in a more explicate way:

$$
\frac{\gamma_{e m}}{\gamma_{e m}^{o}}=\left[\frac{\gamma_{e x c}}{\gamma_{e x c}^{o}}\right]\left[\frac{q}{q^{o}}\right]
$$

where $\gamma_{e m}$ is the emission decay rate, $\gamma_{e x c}$ is the excitation rate, and $q$ is the quantum yield, and the superscript " ${ }^{\prime}$, denote the values without the particle. Following to the Fermi's Golden Rule [Fermi 1950], the excitation decay rate below the optical energy saturation can be expressed in terms of $E^{2}$ :

$$
\frac{\gamma_{e x c}}{\gamma_{e x c}^{o}}=\left|\frac{E}{E^{o}}\right|^{2}
$$

The intrinsic quantum yield of the fluorophore $\left(q^{o}\right)$ is defined to be

$$
q^{o}=\frac{\gamma_{r}^{o}}{\gamma_{r}^{o}+\gamma_{n r}^{o}}
$$

where $\gamma_{r}^{o}$ and $\gamma_{n r}^{o}$ are the radiative decay rate and non-radiative decay rate of the fluorophore without the particle, respectively. When the fluorophore is placed in a plasmon field, both radiative decay rate and non-radiative dacay rate changed.

The expression of the radiative decay rate, $\gamma_{r}$, is derived by computing the radiative power induced by the particle using the polarizability of the particle [Gersten and Nitzan, 1981; Bharadwaj and Novotny, 2007]. For a spherical particle without coating, in vacuum, with quasi-static polarizability, they found

$$
\frac{\gamma_{r}}{\gamma_{r}^{o}}=\left|1+2\left(\frac{r_{1}}{r}\right)^{3} \frac{\varepsilon\left(\omega_{1}\right)-1}{\varepsilon\left(\omega_{1}\right)+2}\right|^{2}
$$


This expression for $\gamma_{r} / \gamma_{r}^{o}$ is identical with the $\left|E / E^{o}\right|^{2}$ for the particle in vacuum, at $\theta=0$, and with incident light frequency of $\omega_{1}$.

The particle also changes the non-radiative decay rate of the fluorophore by adding an additional non-radiative decay rate $\left(\gamma_{a b s}\right)$. This rate can be deduced from the power that is radiated by the fluorophore and absorbed by the particle (for us, GNP). Using the electrostatic image theory [Novotny and Hecht, 2006], the power emitted by the fluorophore and absorbed by the particle inside a half-space can be calculated by integrating the dissipated power density, and the energy transfer rate becomes

$$
\frac{\gamma_{a b s}}{\gamma_{r}^{o}}=\frac{3}{16} \operatorname{Im}\left[\frac{\varepsilon_{1}\left(\omega_{2}\right)-1}{\varepsilon_{1}\left(\omega_{2}\right)+1} \frac{1}{\left(\omega_{2} / c\right)^{3}\left(r-r_{1}\right)^{3}}\right] \frac{\left(p_{x}^{2}+p_{y}^{2}+p_{z}^{2}\right)}{\mid \mathbf{p}^{2}},
$$

where $\omega_{1}$ is the emission light frequency, $c$ is the speed of light and $\mathbf{p}=\left[p_{x}, p_{y}, p_{z}\right]$ is the transition dipole moment. At $\theta=0$,

$$
\frac{\left(p_{x}^{2}+p_{y}^{2}+p_{z}^{2}\right)}{\mid \mathbf{p}^{2}}=2
$$

The intrinsic non-radiative decay rate can be written as $\gamma_{n r}^{o}=\gamma_{r}^{o} \cdot\left(1-q^{o}\right) / q^{o}$. This accounts for nonradiative losses inside the fluorophore only, i.e., it is an intrinsic property of the fluorophore. The quantum yield of a fluorophore affected by the particle then becomes [Bharadwaj, et al., 2007]

$$
q=\frac{\gamma_{r} / \gamma_{r}^{o}}{\gamma_{r} / \gamma_{r}^{o}+\gamma_{a b s} / \gamma_{r}^{o}+\left(1-q^{o}\right) / q^{o}}
$$

In summary, for a selected fluorophore, when the particle size, the coating material and the distance between a fluorophore and a particle are known, the fluorescence change by the particle can be estimated using Eqs 1-15. 
Angers, et al. [2006] and Bharadwaj and Novotny [2007] have used this theory to compute the fluorescence alteration of a fluorophore Nile Blue and a single GNP (60 nm). Their theoretical results correlated well with the experimental results. Cheng, et al., [2011] used DNA strands of various lengths to control the distance between a fluorophore and a GNP of various sizes, and their experimental results also matched well with the theoretical prediction using the model.

\section{(b-ii) Quantum mechanical model}

Gersten and Nitzan [1981] have developed a quantum mechanical model that considered the system with the molecular dipole and the particle's plasmons. The model provides the Hamiltonian and the equations of motion for the system in the presence of the incident radiation field. However, the model is limited to the case when the distance between the fluorophore and the particle is greater than the radius of the particle $[(r$ $\left.r_{l}\right)>r_{l}$ ]. Vukovic, et al. [2009] also developed a model using quantum mechanics. The model considers the chemical structure of the fluorophore, instead of using a point-dipole approximation, and is not limited to single particle, but can address a variable number of particles.

The relative brightness, $\Phi^{\mathrm{RB}}$ is determined by

$$
\Phi^{\mathrm{RB}}=q \cdot\left(A / A_{f r e e}\right)
$$

where $q$ is the quantume yield of the fluorophore as described in Eq. (11), $A_{\text {free }}$ is the molecular absorption coefficient in the absence of the particle. The ratio $A / A_{\text {free }}$ accounts for the different population in the excited state induced by the particle on the molecular absorption. This equation actually has the same physical meaning as in Eqs. (8) and (9). While in computing radiative $\left(\gamma_{r}\right)$ and non-radiative decay rate $\left(\gamma_{n r}\right)$ and the absorption 
coefficient $(A)$, the particle is considered as a continuous dielectric characterized by the optical constants and the fluorescence is treated quantum-mechanically [Corni, et al., 2002; Andreussi, et al., 2004]. The model belongs to a family of the polarizable continuum model (PCM) [Tomasi, et al., 2005]. The fluorophore is described by an effective Hamiltonian. The electrostatic interaction energy between the molecular charge density and the polarization of the particle is induced by the molecular charge density itself. These charges are obtained by the boundary element method solution of the integral equation formalism approach to the Poisson problem [Vukovic, et al., 2009].

The absorption coefficient, $A$, is given by

$$
A=\frac{2 \pi}{3 h^{2} c n}\left|\vec{\mu}_{K 0}\right|^{2}
$$

where $\mathrm{h}$ is the Dirac constant, $n$ is the refractive index of the medium, and $\vec{\mu}_{K 0}$ is the transition dipole between the ground state and the $\mathrm{K}^{\text {th }}$ excited state, and it is directly affected by the interaction with the particle (metal) and the solvent:

$$
\vec{\mu}_{K 0}=\vec{\mu}_{K 0}^{m o l}+\vec{\mu}_{K 0}^{m e t}+\vec{\mu}_{K 0}^{\text {sol }}
$$

where $\vec{\mu}_{K 0}^{m o l}$ is the molecular transition dipole, $\vec{\mu}_{K 0}^{\text {met }}$ and $\vec{\mu}_{K 0}^{\text {sol }}$ are the transition dipole effect of the particle and the solvent, respectively.

The radiative decay rate $\left(\gamma_{r}\right)$ and non-radiative decay rate $\left(\gamma_{n r}\right)$ are

$$
\begin{aligned}
& \gamma_{r}=\frac{4 P^{3} n}{3 h c^{3}}\left|\vec{\mu}_{K 0}\right|^{2} \text { and } \\
& \gamma_{n r}=-2 \operatorname{Im}(\boldsymbol{P}),
\end{aligned}
$$

where $\boldsymbol{P}$ is the excitation energy.

Vokuvic, et al. [2009] used this model (Eq. 16-20) to compute the fluorescence of N,N'-dimethylperylene-3,4,9,10-dicarboximide in combination with silver and gold 
nanoparticles, and the results account for continuous change from quenching to enhancement. However, there have not been many experimental verifications of this model.

\section{NANOPARTICLES FOR BIOMEDICINE}

In 1959, Richard Feynman introduced the concept of nanotechnology in his pioneering lecture titled "There's plenty of room at the bottom" at the annual meeting of the American Physical Society [Appenzeller, 1991]. However, nanotechnology has become a rapidly growing field only in the recent decade. The term "nanotechnology" varies greatly based on the specific definition that is used. The U.S. National Science Foundation and the National Nanotechnology Initiative define the nanotechnology as understanding and control of matter at dimensions of $1-100 \mathrm{~nm}$, where unique phenomena enable novel applications [Khademhosseini and Langer, 2006].

\section{Advantages of nanoparticles for biomedical application}

Nanotechnology is an emerging field that would potentially make a major impact to human health [Khademhosseini and Langer, 2006]. Living organisms are built of cells that are typically in the range of $10 \mu \mathrm{m}$. However, the cell parts are much smaller and are in the sub-micron size domain. The proteins are even smaller, with a typical size of $5 \mathrm{~nm}$. This size comparison reveals that using nanoparticles as probes would allow us to understand the cellular machinery at a molecular level, without introducing too much interference [Taton, 2002; Salata, 2004]. Understanding of biological processes on a nanoscale level has been a strong force behind the development of nanotechnology [Whitesides, 2003]. 
The small size of nanoparticles makes them suitable for bio-tagging or labeling. The surface of a nanoparticle can be conveniently used for a molecular assembly. The molecules are usually one or more than one of the following: The small molecules that make the particle biocompatible, antibodies/aptamers/peptide for biomolecule detection or in vivo targeting, fluorophores for signaling, drugs for disease treatment, etc. Since a particle can carry more than one function in a single entity, multifunctionality is a fundamental characteristic of nanoparticles [Salata, 2004; Singh, et al., 2009]. Studies have also shown that nanoparticles feature a longer circulation time in blood, higher uptake rate by cells [Davis, 2008] and a high concentration of drug in/around them [Ferrari, 2005]. In addition to these general advantages, many nano-materials exhibit unique properties, e.g., optical, magnetic properties, etc., which can be beneficially utilized for biomedicine.

These advantages and special properties of nanoparticles have made nanomedicine a booming research area, especially in cancer diagnosis and therapy. During the past decades, advances in cancer biology have not been translated into medicine appropriately, mainly due to the inability of administering contrast agents and therapeutic moieties selectively to the desired targets [Langer and Tirrel, 2004; Ferrari, 2005]. Nanomaterials may be, therefore, excellent candidates for overcoming these issues.

\section{Types of nanoparticles}

Most of the nanoparticles currently being developed for medical use may be classified into two: i.e., particles of organic molecules mainly and those with the inorganic element, usually metals, as the core. 


\section{(a) Organic nanoparticles}

Organic nanoparticles including liposomes, dendrimers, and other bio-polymers are being developed for drug delivery to various human tumors, including breast cancer [Park, 2002; Hofheinz, 2005]. The first nano-vehicle for drug delivery is liposome. As other nanoparticles, liposomes feature longer circulation time especially when the surface is modified with PEG. With the leaky nature of the cancer neovasculature, they may have a significant selectivity in drug delivery to tumors [Papahadjopoulos, et al., 1991; Oku and Namba, 1994]. Liposome-encapsulated formulations of doxorubicin were approved in the 1990s for the treatment of Kaposi's sarcoma, and are now used to treat breast and refractory ovarian cancer. Liposomes continue to be refined and applied to more cancer types [Langer and Tirrel, 2004; Allen, 2002; Park, 2002; Kircher, et al., 2003; Ferrari, 2005].

Dendrimers and various bio-degradable polymers, such as poly(lactic acid) (PLA), poly (glycolic acid) (PGA), poly (lactic co-glycolic acid) (PLGA) and polyanhydride are also being studied to encapsulate drugs. The particles can be designed to release drugs through the bulk erosion, diffusion, or being triggered by the external environment, such as changes in $\mathrm{pH}$, light, temperature or by the presence of certain biomolecules [Khademhosseini and Langer, 2006; Langer and Tirrel, 2004]. They can also be used to encapsulate highly toxic, poorly soluble or relatively unstable drugs whose clinical use was limited without using nano-capsules [Langer, 1998]. These particles may also include disease-targeting molecules for targeted delivery.

In addition to the drug delivery, nanotechnology has contributed to advance in medical techniques, such as implantable nanoporous membranes [Tao and Desai, 2003], 
controlled release biochips [Santini, et al., 1999], and transdermal microneedle systems [Prausnitz, et al., 2004]. New organic nanoparticles are still being discovered and characterized, providing potential future applications.

\section{(b) Inorganic nanoparticles}

Inorganic nanoparticles are usually a metal or metal compound possessing medically beneficial properties, including size-dependent physical properties, optical and magnetic effects [Salata, 2004].

\section{(b-i) Gold nanoparticles}

Noble metal nanoparticles, such as gold and silver, possess unique optical properties. In biosensing, for years, gold and silver nanoparticles have been used in surface-enhanced Raman scattering (SERS). The sensitivity enhancement factor of SERS is as high as $10^{14}-10^{15}$, which improves the detection limit [Boisselier and Astruc, 2009]. GNPs are used to develop biosensors utilizing the surface plasmon peak shift by their surface or environment change, such as the binding of analytes onto GNP surface conjugated with the recognition molecule, the inter-particle distance changes when GNPs bind to each other via the molecules conjugated on the surface [Jain, et al., 2008].

For bioimaging, GNPs are also studied for many other imaging modalities: Large GNPs $(>20 \mathrm{~nm})$ can be imaged using an optical microscope in the phase contrast or differential interference contrast mode [Boisselier and Astruc, 2009]. Small GNPs absorb light and generate heat which can be detected via the local variation of the refractive index using Differential Interference Contrast (DIC) microscopy or via the heat-induced liquid expansion using photoacoustic imaging [Boisselier and Astruc, 2009]. GNPs can 
also be used in optical coherence tomography (OCT), which is an optical analogue to ultrasound with a relatively good resolution (1-10 $\mu \mathrm{m})$ [Skala, et al., 2008]. GNPs generate an enhanced signal for the multiphoton surface plasmon resonance (SPR) microscopy when they are illuminated at their resonance frequency [Yelin, et al., 2003]. They also generate better contrast in X-ray scattering than the organic contrast molecules, providing high signal-to-noise ratio for the $\mathrm{X}$-ray and $\mathrm{X}$-ray computer tomography [Hainfeld, 2006; Cai, et al., 2007; Kim, et al., 2007]. GNPs are also studied to enhance gamma radiation using neutron activation [Sperling, et al., 2008].

For medical therapies, GNPs are studied for optical cancer hyperthermia (photothermal therapy) [Boisselier and Astruc, 2009]. GNPs have high absorption cross sections requiring less irradiation energy and the irradiation via the SPR of GNPs converts energy from light to heat fast [Hirsch, et al., 2003; O'Neal, et al., 2004; Loo, et al., 2005; Huang, et al., 2006]. NIR is desired for its deep penetration into tissue and the small GNPs (10-30 nm) forming larger clusters absorb NIR light $(700-1000 \mathrm{~nm})$ well [Zharov, 2005]. Gold nanoshells and nanorods that absorb light in NIR are also extensively studied for this purpose [Hirsch, et al., 2003; Loo, et al., 2005].

GNPs are typically synthesized by reduction of chloroauric acid $\left[\mathrm{H}\left(\mathrm{AuCl}_{4}\right)\right]$ using citric acid. They are stabilized by citric acid, but not stable in solutions with a high ionic strength. GNPs for biomedical uses require high stability in physiological conditions, while maintaining their optical properties. Stability, from a biological point of view, means good solubility and dispersivity in the physiological environment, as well as the cellular or molecular interaction [Duchesne, et al., 2008]. The solubility can be achieved by coating the GNP surface with a stable and biocompatible hydrophilic shell. 
Shell materials that have been frequently used are the polymeric capping agents, such as, amino- or mercapto-dextrans or poly ethylene glycol (PEG) (Fig. 3a) and the thiol ligands with hydrophilic end groups, which form a self-assembled monolayer (SAM); (Fig. 3b) [Duchesne, et al., 2008]. Compared to the arbitrary polymer coating, the SAM provides important advantages. The thickness of the coating layer may be kept minimal, which is particularly important for our study with spacers of a short length. It avoids the steric hindrance of the GNP during penetrating into cells or synapses.

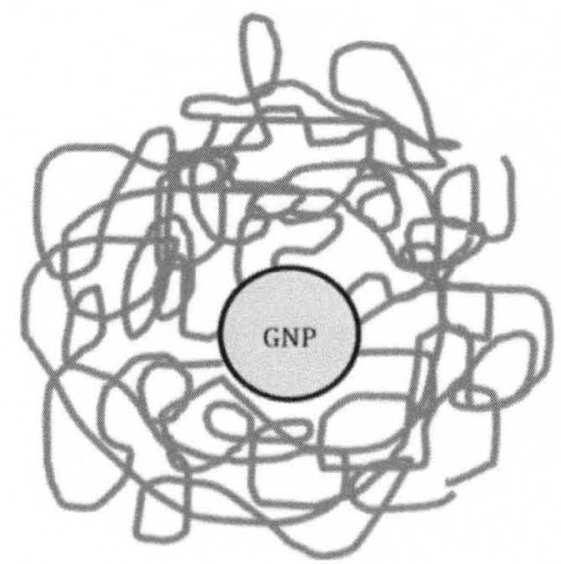

(a)

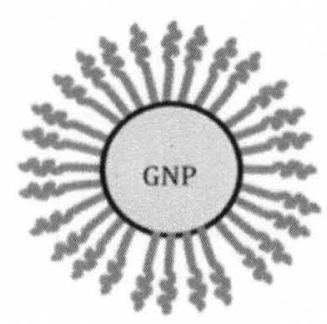

(b)

Figure 3. GNP stabilized with (a) large polyethylene glycol molecules and (b) with small aliphatic-PEG molecules

A number of SAM ligand shells have been developed, and they usually follow a basic design composed of a hydrophobic chain (aliphatic chain), allowing tight packing of the monolayer, and a hydrophilic terminus to provide solubility in physiological environments [Duchesne, et al., 2008; Pciotti, et al., 2006; James, et al., 2007]. The mostused materials for the hydrophilic terminus are PEGs, because PEG layer provides the GNPs with excellent hydrophilicity to the particle, and it minimizes the adsorption of particles to proteins [Ostuni, et al., 2001]. 


\section{(b-ii) Magnetic nanoparticle}

Magnetic nanoparticles (MNP) have recently attracted attention because of their ability of increasing contrast for MRI and cancer hyperthermia. Iron oxide nanoparticles $\left(\mathrm{Fe}_{3} \mathrm{O}_{4}, \mathrm{Fe}_{2} \mathrm{O}_{3}\right)$ are the most explored for these purposes. When the ferrite nanoparticles become smaller than $128 \mathrm{~nm}$ they become superparamagnetic, which prevents selfagglomeration because they exhibit the magnetic property only when the magnetic field is applied [Weissleder, et al., 1990]. They were found to have much greater magnetic susceptibility than traditional MR contrast agents, such as gadolinium [Cuenca, et al., 2006]. They have rapid hepatic uptake after intravenous administration, which makes them useful for the characterization of hepatic tumors [Ferrucci and Stark, 1990]. Ultrasmall-superparamagnetic iron oxide nanoparticles (SPIONs; 5-10 nm) may have better tissue distribution, allowing uptake in lymph nodes and bone marrow [Weissleder, et al., 1990]. They have been used therefore, clinically in human for characterizing lymph node status in patients with cancers [Cuenca, et al., 2006].

MNPs can be heated in an alternating electro-magnetic (AEM) field, by hysteresis loss, Neel relaxation, or/and Brownian relaxation [Hergt, et al., 1998]. Therefore, they are extensively studied for localized cancer hyperthermia [Jin, et al., 2006; Kumar and Mohammad, 2011]. Compared to the traditional hyperthermia treatment, MNPs can be targeted for the cancer by using the disease-specific binding agents, making the treatment tumor - ppecific. The AEM frequencies utilized are harmless to the body and generate heat only in the tumor containing MNPs [Rand, et al., 1985]. This hyperthermia method can be combined with controlled delivery of drugs, utilizing heat-induced bond-breaking 
between drug and polymer on the MNP, heat-enhanced permeability of the drug from the polymer to the tumor, etc. [Kumar and Mohammad, 2011].

Paramagnetic nanoparticles have also been used in concentrated drug delivery to tumors [Cuenca, et al., 2006]. Alexiou, et al. [2000] used magnetic fields to concentrate methotrexate-conjugated nanoparticles in tumor to focus greater amounts of the drug in the desired areas, while being able to lower the systemic or intra-arterial concentration. This drug delivery system was translated into a Phase I clinical trial and demonstrated better patient tolerance of therapy with the successful localization of nanoparticles to the tumors but with no magnetic field-associated toxicities [Lubbe, et al., 1996].

Nanoparticles with a magnetic core, either of elementary Iron or Cobalt, with nonreactive shell have been synthesized recently for biomedical research application [Grass and Stark, 2006]. Magnetic CoPt nanoparticles and iron-oxide-based magnetic materials have been used in clinical practice as contrast agents for MRI [Meng, et al, $2011]$

MNP may also be coated with a gold layer to incorporate the optical properties of gold and achieving multi-functions for disease diagnosis and treatment [Chen, et al., 2003].

In general, nanoparticles features numerous advantages that can be beneficially applied to biomedicine. Although this study focuses only on the unique phenomenon of fluorescence manipulation by GNP, the GNP can be a platform to incorporate many other beneficial functions in the future. 


\section{E. FLUORESCENCE ALTERATION BY GNP, PREVIOUSLY STUDIED BY THE KANG GROUP}

The Kang group has been studying the application of GNPs for fluorophoremediated biosensing and bioimaging since 2005. Hong and Kang [2006] applied GNPs to a fiber-optic immuno-biosensing system to increase its sensitivity. The sensor performs a fluorophore-mediated sandwich immunoassay on the sensor surface, and the fluorescence intensity is correlated with the analyte concentration [Kang et al., 1997; Spiker et al., 1998]. Fluorolink Cyanine 5 (Cy5, Ex/Em 647/679 nm) and Alexa Fluor 647 (AF647; $\mathrm{Ex} / \mathrm{Em} 649 / 666 \mathrm{~nm}$ ) were used as the fluorophore. To adjust the distance between the free GNP and fluorophores in solution, self-assembled monolayers (SAMs) at various thicknesses were coated on the gold particle surface. The effect of GNP size and the SAM thickness on fluorescence enhancement was studied.

Hong and Kang [2006] also found that some solvents can enhance the fluorescence of a fluorophore, by shifting the fluorophore excitation/emission wavelengths [Buschmann, et al., 2003], and/or by changing the trans-cis isomerization direction [Aramendia, et al., 1994; Scaffardi, et al., 1997; Rodríguez, et al., 1997], and/or by shrinking proteins if fluorophores are tagged to them [Bonincontro et al., 1997; Ruckebusch et al., 1999]. They have found that 1-butanol and ethanol gave high enhancement without denaturing the antibodies immobilized on the sensor surface. To maximize the enhancement effect, the two enhancers, the GNP-SAM and the solvent were mixed to form GNP reagent (GNPR). Hong, et al. [2006] and Wang, et al. [2008] applied the GNPR in Alexa fluorophore (Alex-647) mediated four-cardiac marker sensing system to be used for real-time diagnosis of acute myocardial infarction. By 
using GNPR, the size of the sensors was reduced from $3 \mathrm{~cm}$ to $1.5 \mathrm{~nm}$, enabling a portable, mini-sensing chip $(2 \times 2.5 \mathrm{~cm})$, and this system can accurately quantify four cardiac markers simultaneously at clinically significant concentration range within 15 min, requiring a plasma sample volume of only $200 \mu \mathrm{l}$.

In biosensing the distance between the GNP and the fluorophore may not be precisely controlled because only the minimum distance between these two may be controlled by the thickness of the SAM. The distance may also be strongly affected by the GNP concentration, because the reagents are confined in a small sensing channel [Wang, et al., 2008]. However, this problem may be resolved by testing the agent for the controlled sensing protocal.

Unlike in the biosensing, for bioimaging in vivo, the fluorophore needs to be physically linked to the GNP and the distance between the GNP and the fluorophore needs to be well controlled. Kang, et al. [2011] have studied the fluorescence change of an NIR fluorophore, Cypate, with changing the distance from a $10 \mathrm{~nm}$ GNP. The distance was controlled by coating a polymer layer at known thickness on the GNP. Cypate was placed on the outermost layer of the polymer coated GNPs, artificially separated from the GNP at the distance of the polymer layer thickness, and its fluorescence levels were observed. The fluorescence of Cypate on the particle surface was quenched almost completely and, at approximately $5 \mathrm{~nm}$ from the surface, it was enhanced $\sim 17$ times. Then the level went down thereafter. This study experimentally suggested the relationship between the level of Cypate fluorescence alteration and the distance from a GNP. 
To have a thorough understanding of fluorescence alteration by GNP, this dissertation theoretically studies the fluorescence of various fluorophores affected by GNPs. In addition, experimentally, a novel, highly specific and sensitive contrast agent for bioimaging is developed utilizing fluorescence alteration by GNP. 


\section{CHAPTER III}

\section{MATERIALS, INSTRUMENTS, AND METHODS}

\section{A. THEORETICAL STUDY}

The fluorophores selected for our study are the ones frequently used in biomedical studies and at various Em/Ex wavelengths. They are Fluorescein isothiocyanate (FITC), Alexa Fluor 532 (AF532), Cyanine 3 (Cy3), Alexa Fluor 647 (AF647), Alexa Fluor 700 (AF700) and Indocyanine Green (ICG). The fluorophores, their Ex/Em peak wavelengths and intrinsic quantum yield values are listed in Table 1.

Table 1. Fluorophores studied, and their excitation/emission peak wavelengths and intrinsic quantum yields.

\begin{tabular}{cccc}
\hline Fluorophore & $\begin{array}{r}\text { Excitation peak } \\
\text { wavelength (nm) }\end{array}$ & $\begin{array}{c}\text { Emission peak } \\
\text { wavelength (nm) }\end{array}$ & $\boldsymbol{q}^{\mathbf{o}}$ \\
\hline FITC & 494 & 521 & 0.93 \\
AF 532 & 530 & 555 & 0.61 \\
Cy3 & 550 & 570 & 0.15 \\
AF 647 & 650 & 665 & 0.33 \\
AF 700 & 702 & 723 & 0.25 \\
ICG (Cypate) & 780 & 830 & 0.012 \\
\hline
\end{tabular}

Solutions for the mathematical model were computed using Matlab R2008a (The Mathworks Inc., Natick, MA). The dielectric permittivity values used in the plasmon field strength computation are: for GNPs, the experimental data by Johnson and Christy [1972] were used; for the shell, value for polyethylene glycol (PEG), $2.85 \varepsilon_{0}$, was used, 
where $\varepsilon_{\mathrm{o}}$ is the dielectric constant of vacuum, $8.85 \times 10^{-12} \mathrm{C}^{2} / \mathrm{N} \mathrm{m}^{2}$. For the surrounding medium, water, the $\varepsilon_{3}$ value of $1.76 \varepsilon_{0}$ was used.

\section{B. SYNTHESES OF SPACERS AND MODIFICATION OF CYPATE}

In this section, the materials, instruments and methods of making the spacers, SSP-Cy and m-Cypate are presented.

The molecular spacers tested for Cypate fluorescence alteration were synthesized by the Nantz group at the University of Louisville or by the NeoGroup Inc. (Cambridge, MA) through customized peptide synthesis, with our design. The detailed structures of the spacers are listed in Table 2.

Table 2. Molecular spacers used for conjugating Cypate to GNPs.

\begin{tabular}{|c|c|c|c|}
\hline Catagory & Structure & Abbreviation & Producer \\
\hline \multirow{2}{*}{ Short spacer } & SH- $\left(\mathrm{CH}_{2}\right)_{2}$-Gly-Gly-Arg-Gly-Gly-Gly-NH & SSP & \multirow{2}{*}{ The Nantz group } \\
\hline & $\mathrm{SH}$ - $\left(\mathrm{CH}_{2}\right)_{2}$-Gly-Gly-Arg-Gly-Gly-Gly-ONH & $n S S P$ & \\
\hline \multirow{5}{*}{ Long spacer } & $\mathrm{HS}-\left(\mathrm{CH}_{2}\right)_{12}-\left(\mathrm{OCH}_{2} \mathrm{CH}_{2}\right)_{4}-\mathrm{ONH}_{2}$ & $\begin{array}{c}\text { LSP } \\
(\mathrm{C} 12 \mathrm{PEG} 4)\end{array}$ & \multirow{5}{*}{ The Nantz group } \\
\hline & $\mathrm{HS}-\left(\mathrm{CH}_{2}\right)_{12}-\left(\mathrm{OCH}_{2} \mathrm{CH}_{2}\right)_{6}-\mathrm{ONH}_{2}$ & $\begin{array}{c}L \mathrm{SP} \\
(\mathrm{C} 12 \mathrm{PEG} 6)\end{array}$ & \\
\hline & $\mathrm{HS}-\left(\mathrm{CH}_{2}\right)_{12}-\left(\mathrm{OCH}_{2} \mathrm{CH}_{2}\right)_{8}-\mathrm{ONH}_{2}$ & $\begin{array}{c}\text { LSP } \\
\text { (C12PEG8) }\end{array}$ & \\
\hline & $\mathrm{HS}-\left(\mathrm{CH}_{2}\right)_{16}-\left(\mathrm{OCH}_{2} \mathrm{CH}_{2}\right)_{4}-\mathrm{ONH}_{2}$ & $\begin{array}{c}L S P \\
(\mathrm{C} 16 \mathrm{PEG} 4)\end{array}$ & \\
\hline & $\mathrm{HS}-\left(\mathrm{CH}_{2}\right)_{16}-\left(\mathrm{OCH}_{2} \mathrm{CH}_{2}\right)_{6}-\mathrm{ONH}_{2}$ & $\begin{array}{c}L S P \\
(\mathrm{C} 16 \mathrm{PEG} 6)\end{array}$ & \\
\hline Dual spacer & $\begin{array}{l}\text { cyclo[DAP(Aoa)-PEG5-(8-aminooctanoic } \\
\text { acid)-Cys-Gly-Arg-Gly-Gly-Gly]* }\end{array}$ & $r D S P$ & NeoGroup Inc. \\
\hline
\end{tabular}

* The molecular structure of $r D S P$ is shown in Fig. 33.

To enable the oxime coupling of Cypate to the nSSP and LSPs, The Nantz group added aldehyde groups to the Cypate (modified-Cypate; m-Cypate). 


\section{B-1. MATERIALS}

Polystyrene resin and amino acids used in the synthesis of short and long spacers were purchased from Novabiochem (San Diego, CA). The coupling agents, 3(diethoxyphosphoryloxy)-1,2,3-benzotriazin-4(3H)-one (DEPBT), hydroxybenzotriazole (HOBt), N,N-Diisopropylethylamine (DIPEA), $\mathrm{CH}_{2} \mathrm{Cl}_{2}$, trifluoroacetic acid and triethylsilane were obtained from Aldrich (St. Louis, MO). All other materials were purchased from Sigma Aldrich (St. Louis, MO), unless otherwise specified.

Cypate (Cy) was produced and supplied by The Achilefu group at the Department of Radiology, Washington University School of Medicine, St. Louis, MO [Achilefu, et al., 2002; Ye, et al., 2003].

\section{B-2. INSTRUMENTS}

For purifying the spacers after each reaction silica gel flash column chromatography (Teledyne ISCO; Lincoln, NE) was used. Solvent evaporation was done using a bench top solvent purifier SPBT-1 (LC Technology Solutions, Inc.) Synthesized SPs was analyzed by HPLC (Waters Corporation Delta 600 instrument; Waters corp.; Milford, MA) and high-resolution mass spectrometry (HRMS; LTQ FT Ultra mass spectrometer; Thermo Scientific; Waltham, MA) with TriVersa NanoMate system chipbased infusion (Advion Biosciences, Inc.; Ithaca, NY).

The theoretical length estimations of the spacers and other molecules were done using the computer software Hyperchem 7.5 (Hypercube Inc.; Gainesville, FL). 


\section{B-3. METHODS}

The methods described in this section are the revised version of the project report from the Nantz group.

\section{(a) Synthesis of the short spacers}

The short spacer, $\mathrm{HS}-\mathrm{CH}_{2}-\mathrm{CH}_{2}-\mathrm{G}-\mathrm{G}-\mathrm{R}-\mathrm{G}-\mathrm{G}-\mathrm{G}-\mathrm{NH}_{2}(\mathrm{SSP})$ was synthesized using standard solid-phase peptide synthesis techniques [Merrifield, 1963]. For $\mathrm{HS}-\mathrm{CH}_{2}-\mathrm{CH}_{2}-$ G-G-R-G-G-G-ONH ${ }_{2}$ (nSSP), the $\mathrm{HS}-\mathrm{CH}_{2}-\mathrm{CH}_{2}-\mathrm{G}-\mathrm{G}-\mathrm{R}-\mathrm{G}-\mathrm{G}-\mathrm{NH}_{2}$ was synthesized following the same methods used for SSP. Then $100 \mathrm{mg}$ of the resin loaded with it was swelled using Dimethylformamide (DMF) over 30 mins, filtered, and then treated with

$0.21 \mathrm{mmol}$ Fmoc-protected aminooxyglycine, $0.21 \mathrm{mmol}$ DIC, $0.21 \mathrm{mmol}$ Hydroxybenzotriazole (HOBt) and $0.42 \mathrm{mmol}$ Diisopropylethylamine (DIPEA) in $2 \mathrm{~mL}$ of DMF. After shaking the mixture overnight, the resin was filtered and then thoroughly washed with $\mathrm{CH}_{2} \mathrm{Cl}_{2} 7$ times. The resin was dried under vacuum, swelled using dimethylformamide, filtered, and then treated with $20 \%$ piperidine in $3 \mathrm{ml} \mathrm{DMF}$ at room temperature for 40 mins. The piperidine washing process was repeated once. The resin was filtered and washed successively with DMF for 3 times and with $\mathrm{CH}_{2} \mathrm{Cl}_{2}$ for 5 times, and then dried under vacuum.

\section{(b) Conjugation of Cypate to SSP}

0.227 mmol Cypate was added to the $\mathrm{HSCH}_{2} \mathrm{CH}_{2} \mathrm{NH}-\mathrm{G}-\mathrm{G}-\mathrm{R}-\mathrm{G}-\mathrm{G}-\mathrm{G}-\mathrm{NH}_{2}$-loaded resin (65 $\mathrm{mg}, 0.045 \mathrm{mmol}$ peptide) in DMF at room temperature. $0.227 \mathrm{mmol}$ DEPBT, $0.227 \mathrm{mmol} \mathrm{HOBt}$, and $0.45 \mathrm{mmol}$ DIPEA were added as coupling agents. After shaking the mixture overnight, the resin was filtered and thoroughly washed with $\mathrm{CH}_{2} \mathrm{Cl}_{2} 7$ times. The resin was then dried under vacuum. To the $\mathrm{HS}-\mathrm{CH}_{2}-\mathrm{CH}_{2}-\mathrm{NH}-\mathrm{G}-\mathrm{G}-\mathrm{R}-\mathrm{G}-\mathrm{G}-\mathrm{G}-$ 
$\mathrm{NHC}(\mathrm{O})$-Cypate resin prepared above $(15 \mathrm{mg}, 0.01 \mathrm{mmol})$ in $0.3 \mathrm{~mL}$ of $\mathrm{CH}_{2} \mathrm{Cl}_{2}, 0.06 \mathrm{~mL}$ of triethylsilane was added at room temperature. After shaking the mixture for $15 \mathrm{~min}$, $0.04 \mathrm{~mL}$ of trifluoroacetic acid was added and the resin suspension was shaken for $1 \mathrm{hr}$, allowed to stand for $1 \mathrm{hr}$, and then shaken for $3 \mathrm{hrs}$. The resin was filtered and the filtrate was concentrated by rotary evaporation. The residue was washed with diethylether for 5 times and dried under vacuum to obtain the target cysteamine-hexapeptide-cypate (SSPCy) conjugate. The conjugates were characterized with HRMS (FTICR).

\section{(c) Synthesis of long spacers}

Scheme 1 illustrates the procedure for the synthesis of $\mathrm{HS}-\left(\mathrm{CH}_{2}\right)_{12}-\left(\mathrm{OCH}_{2} \mathrm{CH}_{2}\right)_{8}-$ $\mathrm{ONH}_{2}$, as an example of the synthesis of long spacers.

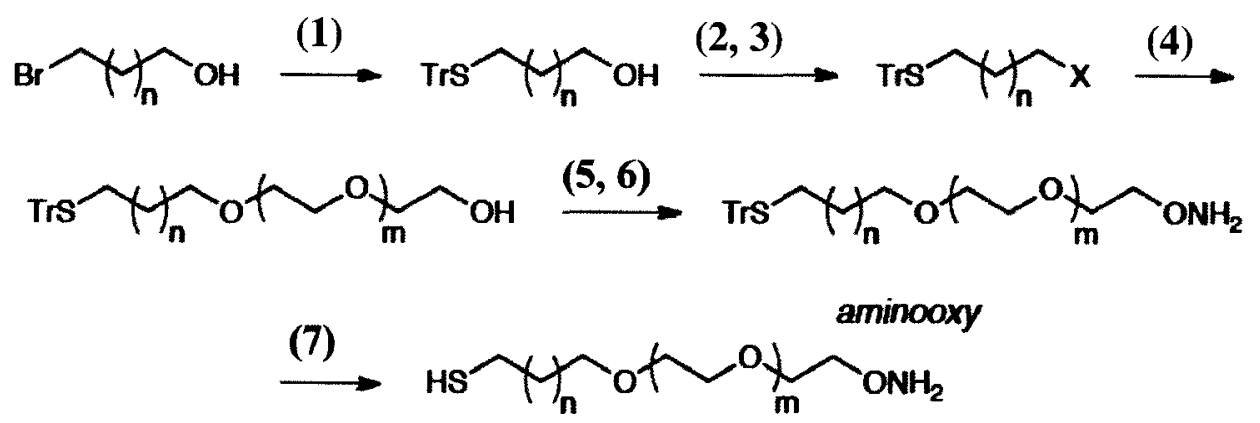

Scheme 1. Synthesis route of the spacers

The sequence number in the content before corresponds to the numbers in Scheme 1. (1) $7.56 \mathrm{mmol}$ Potassium carbonate was added to a solution of $3.78 \mathrm{mmol} 12$-bromododecanol in methanol, at room temperature. After stirring it for 5 minutes, 4.90 mmol triphenylmethanethiol $(\mathrm{TrS})$ was added, and then the mixture was stirred for 16 hours. It was then diluted with diethyl ether and neutralized with 
$\mathrm{Na}_{2} \mathrm{HPO}_{4} / \mathrm{NaH}_{2} \mathrm{PO}_{4}$ buffer. The buffer was removed, and the organic layer was washed with $\mathrm{NaCl}$ brine 3 times. The aqueous layers were combined and extracted with diethyl ether, dried over $\mathrm{Na}_{2} \mathrm{SO}_{4}$ and concentrated by rotary evaporation. The crude residue $\left[\mathrm{TrS}-\left(\mathrm{CH}_{2}\right)_{12}-\mathrm{OH}\right]$ was purified by flash chromatography.

(2) $30 \mathrm{~mL}$ dry $\mathrm{CH}_{2} \mathrm{Cl}_{2}$ was added to a $\mathrm{N}_{2}$ purged flask containing $1.95 \mathrm{mmol}$ $\operatorname{TrS}\left(\mathrm{CH}_{2}\right)_{12} \mathrm{OH}$ and equal mole of sodium bicarbonate.

(3) $4.88 \mathrm{mmol}$ carbon tetrabromide and $5.07 \mathrm{mmol}$ triphenylphosphine in minimal dry $\mathrm{CH}_{2} \mathrm{Cl}_{2}$ were added, and stirred for 12 hours. The resulting solution [TrS$\left.\left(\mathrm{CH}_{2}\right)_{12}-\mathrm{Br}\right]$ was washed, extracted, dried and purified as described in the step (1).

(4) $6.18 \mathrm{mmol}$ tetra(ethylene glycol) in DMF was added to $12.17 \mathrm{mmol}$ sodium hydride in $\mathrm{DMF}$, at $0^{\circ} \mathrm{C}$ under $\mathrm{N}_{2}$. After 5 minutes, $1.03 \mathrm{mmol} \operatorname{TrS}\left(\mathrm{CH}_{2}\right)_{12} \mathrm{Br}$ in DMF was added, and then allowed to reach room temperature and stirred under $\mathrm{N}_{2}$ for 72 hours. The product $\left[\mathrm{TrS}-\left(\mathrm{CH}_{2}\right)_{12}-\left(\mathrm{OCH}_{2} \mathrm{CH}_{2}\right)_{8}-\mathrm{OH}\right]$ was then washed, extracted, dried and purified.

(5) $0.414 \mathrm{mmol} \mathrm{N}$-hydroxyphthalimide and the same mole of triphenylphosphine were added to $0.345 \mathrm{mmol} \operatorname{TrS}-\left(\mathrm{CH}_{2}\right)_{12}-\left(\mathrm{OCH}_{2} \mathrm{CH}_{2}\right)_{8}-\mathrm{OH}$ in dry THF, under $\mathrm{N}_{2}$ at $0^{\circ} \mathrm{C}$. Then $0.414 \mathrm{mmol}$ diisopropyl azodicarboxylate was added dropwise. The solution was warmed to room temperature and stirred overnight. The mixture was concentrated by rotary evaporation, and washed, extracted, dried and purified $\left[\mathrm{TrS}\left(\mathrm{CH}_{2}\right)_{12}-\left(\mathrm{OCH}_{2} \mathrm{CH}_{2}\right)_{8}-\mathrm{ONPhth}\right]$.

(6) $3.35 \mathrm{mmol}$ hydrazine monohydrate was added to a solution of $0.168 \mathrm{mmol}$ $\operatorname{TrS}\left(\mathrm{CH}_{2}\right)_{12} \mathrm{OPEG} \mathrm{ONPhth}_{8} \mathrm{On} \mathrm{CH}_{2} \mathrm{Cl}_{2}$, at room temperature, and the mixture was 
stirred for 3 hours. The product $\left[\mathrm{TrS}\left(\mathrm{CH}_{2}\right)_{12}-\left(\mathrm{OCH}_{2} \mathrm{CH}_{2}\right)_{8}-\mathrm{ONH}_{2}\right]$ was dried by rotary evaporation and purified by flash chromatography.

(7) Excessive amount of trifluoroacetic acid and $0.073 \mathrm{mmol}$ triethylsilane were added to a solution of $0.123 \mathrm{mmol} \operatorname{TrS}\left(\mathrm{CH}_{2}\right)_{12} \mathrm{OPEG}_{8} \mathrm{ONH}_{2}$ in $\mathrm{CH}_{2} \mathrm{Cl}_{2}$, at room temperature and the mixture was stirred for 3 hours under $\mathrm{N}_{2}$. The product [HS$\left.\left(\mathrm{CH}_{2}\right)_{12}-\left(\mathrm{OCH}_{2} \mathrm{CH}_{2}\right)_{8}-\mathrm{ONH}_{2}\right]$ was dried by rotary evaporation and vacuum pumping, and then purified by flash chromatography.

Other spacers, $\mathrm{HS}-\left(\mathrm{CH}_{2}\right)_{\mathrm{n}}-\mathrm{PEG}_{\mathrm{m}}-\mathrm{ONH}_{2}$ for $\mathrm{n}=12$ and 16 , and $\mathrm{m}=4$ and 6 , were synthesized following the same procedure. Each product was analyzed and confirmed by HPLC and HRMS.

\section{(d) Modification of Cypate}

In this reaction, $\left(\mathrm{C}_{2} \mathrm{H}_{5} \mathrm{O}\right)_{2} \mathrm{CH}\left(\mathrm{CH}_{2}\right)_{3} \mathrm{NH}_{2}$ was conjugated to the two $-\mathrm{COOH}$ groups of Cypate via the amine-carboxylic acid reaction, and the $-\mathrm{CH}\left(\mathrm{OC}_{2} \mathrm{H}_{5}\right)_{2}$ group was then modified to $-\mathrm{CHO}$.

(1) $0.48 \mathrm{mmol}$ amino acetal $\left(\mathrm{C}_{2} \mathrm{H}_{5} \mathrm{O}\right)_{2} \mathrm{CH}\left(\mathrm{CH}_{2}\right)_{3} \mathrm{NH}_{2}$ was added to a mixture of 0.12 mmol Cypate, $0.48 \mathrm{mmol} \mathrm{HOBt}$, and $0.48 \mathrm{mmol}$ EDC in $2 \mathrm{~mL}$ dry DMF, at room temperature, and the mixture was stirred overnight in dark, at room temperature.

(2) The step (1) was stopped by adding water, and the product [Cypate conjugated with two molecules of $\left.\left(\mathrm{C}_{2} \mathrm{H}_{5} \mathrm{O}\right)_{2} \mathrm{CH}\left(\mathrm{CH}_{2}\right)_{3} \mathrm{NH}_{2}\right]$ was extracted using $\mathrm{CH}_{2} \mathrm{Cl}_{2}$. The organic layer was washed with brine and then dried with $\mathrm{Na}_{2} \mathrm{SO}_{4}$. The solvents were removed by rotary evaporation and the residue was purified by column chromatography $\left(\mathrm{SiO}_{2}\right.$, eluting with a gradient of $1-7 \%$ methanol in $\left.\mathrm{CH}_{2} \mathrm{Cl}_{2}\right)$. 
(3) The product was hydrolyzed by dissolving it at $1.6 \mathrm{mg} / \mathrm{ml}$ in $30 \%$ aqueous acetic acid, with stirring for $3.5 \mathrm{~h}$ at room temperature. The solvent was then removed under vacuum to obtain aldehyde-Cypate (modified Cypate; mCy) as a green solid.

\section{CONJUGATION OF CYPATE TO GNP}

\section{C-1. MATERIALS}

Gold nanoparticles (GNPs; 5, 10, and $15 \mathrm{~nm}$ according to the manufacture's information; 3.7, 8.0 and $16.4 \mathrm{~nm}$ by our measurement) stabilized with citric acid and tannic acid in aqueous solution were purchased from Ted Pella (Redding, CA).

The GNP coating material (1-Mercapto-11-undecyl) tetra(ethylene glycol) [HS$\left(\mathrm{CH}_{2}\right)_{11}-\left(\mathrm{CH}_{2} \mathrm{CH}_{2} \mathrm{O}\right)_{3} ; \mathrm{MW}, 380.58$; sPEG] was from Asemblon (Redmond, WA) or ProChimia Surfaces (Ul. Zacisze, Poland).

Urokinase-type plasminogen activator (uPA) was purchased from Innovative Research (Novi, MI).

Pure argon was provided by Welders Supply (Louisville, KY) to blow-dry the substrate; ethanol, dimethyl sulfoxide (DMSO) and all other chemicals were from SigmaAldrich, unless otherwise specified.

\section{C-2. INSTRUMENTS}

For GNP purification after conjugation, dialysis was done with Slide-A-Lyzer Dialysis Cassettes (20K MW Cut-Off; Thermo Scientific; Rockford, IL). Centrifugation was done using Eppendorf 5415 R Centrifuge (Eppendorf AG; Hamburg, Germany). A 
sonicator (Sonic Dismembrator; Fisher Scientific; Chicago, IL) was used to disperse GNPs pellet in solutions after centrifugation.

For characterizating various GNP products, absorption spectra were used (Beckman DU520 spectrometer; Beckman Instruments, Inc.; Fullerton, CA). The size of GNPs before and after the surface modification was measured by a dynamic light scattering (DLS) particle size analyzer (90Plus/BI-MAS; Brookhaven Instruments Co.; Holtsville, NY) and a transmission electron microscope (TEM; Tecnai ${ }^{\mathrm{TM}}$ HR FEG-TEM; FEI co.; Hillsboro, Oregon).

Fluorescence was measured by the Spectra Gemini XPS fluorometer (Molecular Devices Corp.; Sunnyvale, CA) in the 96-well Uniplate (Whatman; Florham Park, NJ) at the excitation and emission wavelengths of 780 and $830 \mathrm{~nm}$, respectively.

\section{C-3. METHODS}

\section{(a) Handling of thiolated materials:}

The GNP surface coating materials (sPEG) and all spacers (SSP-Cy, $n S S P, L S P s$, and $r D S P$ ) reacting to GNPs contain a thiol ending, which can be oxidized easily by oxygen in the air. Therefore, special care is required in handling these spacers. The spacers were received in a solid form at room temperature. To dissolve the spacers ethanol was first purged with argon. The spacers were dissolved at a concentration of $\sim 5$ $\mathrm{mg}$, and the solution was divided into small aliquots, sealed in containers under argon, and kept at $-70{ }^{\circ} \mathrm{C}$ for a short-term $(<2$ month). For a long-term storage, the spacer was blow-dried with argon to form a solid and the container was sealed under argon, and kept at $-70^{\circ} \mathrm{C}$. 
Pure sPEG is solid at $4^{\circ} \mathrm{C}$ and becomes liquid at room temperature. At room temperature it is made into small aliquots and then kept under argon, at $4^{\circ} \mathrm{C}$ as a solid as suggested by the manufacture. Before each use, sPEG was warmed to room temperature and $2 \mu \mathrm{L}$ of sPEG was taken and dissolved in $500 \mu \mathrm{L}$ ethanol, forming stock solution at $3.4 \mathrm{mg} / \mathrm{mL}(9390 \mu \mathrm{M})$.

\section{(b) GNP surface modification and Cypate conjugation}

\section{(b-i) Conjugation of SSP-Cy to GNP}

SSP-Cy and sPEG were conjugated to GNP following the procedure described below:

(1) SSP-Cy and sPEG were mixed at a molar ratio of 1:4 and diluted in ethanol in a volume equal to the volume of the GNP solution to be added, to result in a $1: 1$ (volume ratio) ethanol and water mixture.

(2) The colloidal GNP solution was added to the SSP/sPEG mixture and the mixture was stirred for $4 \mathrm{hrs}$ at room temperature. According to Duchesne, et al. [2002], a tightly packed, mixed monolayer of sPEG and peptide on the GNP surface corresponds to 3.6 molecules $/ \mathrm{nm}^{2}$-GNP surface area. Here, the amount of SSP-Cy and SPEG was at 60 molecules $/ \mathrm{nm}^{2}$-GNP surface area (excess by 15 times).

(3) After the reaction, the solution was placed in a dialysis cassette and the cassette was placed in $2 \mathrm{~L}$ of de-ionized (DI) water in dark with stirring overnight.

(4) The dialyzed sample was then centrifuged at 13000 RPM for $60 \mathrm{~min}$. The pellet was re-dispersed in $1 \mathrm{~mL}$ of $\mathrm{DI}$ water and sonicated for 5 minutes. 
(5) The GNP and Cypate in the solution were quantified by their respective absorption peaks (520 and $780 \mathrm{~nm}$, respectively).

\section{(b-ii) Conjugation of $n S S P$ to GNP/ Conjugation of mCy to $n S S P$ on GNP}

$n S S P$ and sPEG were first reacted onto GNP and purified, and then m-Cypate was reacted to GNP-nSSP:

(1) $n S S P$ and sPEG were mixed at a ratio of 1:4 and diluted in ethanol by the same method described in step (1-3) of (b-i) above.

(2) The GNP was quantified by the light absorption at $520 \mathrm{~nm}$.

(3) $\mathrm{mCy}$ was dissolved in ethanol at $2.5 \mathrm{mM}$; a blocking agent, $\mathrm{Me}_{3} \mathrm{~N}^{+}-\left(\mathrm{CH}_{2}\right)_{2}-\mathrm{ONH}_{2}$ (BLK) was added to $\mathrm{mCy}$ solution at a 1:1 molar ratio and reacted in dark, at room temperature, overnight. Addition of BLK is to block one of the two reactive $-\mathrm{CHO}$ groups of $\mathrm{mCy}$, and increases the hydrophilicity of $\mathrm{mCy}$.

(4) mCy-BLK was added to the solution from step (2) at 0.3 molecule/nm²-GNP surface and reacted with stirring, for $4 \mathrm{hrs,} \mathrm{in} \mathrm{dark,} \mathrm{at} \mathrm{room} \mathrm{temperature.}$

(5) The solution from step (4) was dialyzed using the cassette in $2 \mathrm{~L}$ of DI water with stirring, in dark, overnight.

(6) The dialyzed sample was then centrifuged at 13000 RPM for $60 \mathrm{~min}$. The pellet was re-dispersed in $1 \mathrm{~mL}$ of $\mathrm{DI}$ water and sonicated for 5 minutes.

(7) The GNP and m-Cypate in the solution were quantified spectroscopically. 


\section{(b-iii) Conjugation of m-Cypate to GNP via long spacers ( $L S P$ )}

LSP and sPEG were first reacted onto GNP and purified, and then m-Cypate was reacted to GNP-LSP.

(1) Colloidal GNP solution was concentrated $\sim 10$ times by centrifugation at 13,000 RPM for 60 mins;

(2) The GNP concentration was determined by the absorption at $520 \mathrm{~nm}$;

(3) To avoid aggregation of the GNP and crowding of mCy on the GNP surface, $L S P$ and sPEG were mixed at a molar ratio of $1: 9$, and the mixture with GNPs in water, for $4 \mathrm{hrs}$ at room temperature. For the amount of $L \mathrm{SP} / \mathrm{sPEG}, 60$ molecule/nm ${ }^{2}$-GNP surface area (excess by $\sim 15$ times) was used;

(4) The purification of GNP-sPEG/LSP, preparation of mCy-BLK, the conjugation of mCy-BLK to GNP-sPEG/LSP, and the purification and quantification of the final product were done by the same way as described in section (b-ii).

\section{(b-iv) Conjugation of $r D S P$ to GNP and the conjugation of $\mathrm{mCy}$ to GNP-rDSP}

$r D S P$ and sPEG were mixed at 1:9 and diluted in ethanol at the same volume of the GNP solution to be added; the rest of the steps were the same as steps (2-8) in section (b-ii).

\section{CELL STUDIES}

\section{D-1. MATERIALS}

The human breast cancer cell lines, MDA-MB-231 and MCF-7, were purchased from American Type Culture Collection (Manassas, VA). Dulbecco's Modified Eagle 
Medium (DMEM), Penicillin and Streptomycin were purchased through Invitrogen (Carlsbad, CA).

Enzyme-linked immunosorbent assay (ELISA) kit for uPA was purchased from Innovative Research (Novi, MI).

\section{D-2. INSTRUMENTS}

For the cell studies performed in the Achilefu group, the cells were plated on LabTek slides (BD Biosciences, Franklin Lakes, NJ). The fluorescence of media was measured in a Pearl Imager (LI-COR, Lincoln, NE). Fluorescence of cells was visualized with an Olympus FV1000 confocal microscope (Olympus America Inc.; Center Valley, PA) using a 20X water immersion objective, and fluorescence intensity was quantified with a built-in photon counter. Before any GNP product is applied on the cell culture they were sonicated using a sonicator (Sonic Dismembrator; Fisher Science, Chicago, IL) to avoid any aggregation.

For the ELISA of uPA, to mix the reagents in a 96-well plate, the Titer Plate Shaker was used (Millipore; Billerica, MA). The fluorescence was measured using a Spectra Gemini XPS fluorometer (Molecular Devices Corp.; Sunnyvale, CA) in the 96well Uniplate (Whatman; Florham Park, NJ).

\section{D-3. METHODS}

(a) Quantification of uPA in cell media by ELISA

MDA-MB-231 and MCF-7 cells were plated at the density of 2500 cells/well (+/$1 \%$ error in pipetting) in serum-free DMEM Media $(150 \mu \mathrm{L} /$ well $)$, in a 96-well plate in the Bates laboratory. For MCF-7 cells, $0.01 \%$ Insulin was added. 
The plate was incubated in $\mathrm{CO}_{2}$ incubator at $37{ }^{\circ} \mathrm{C}$. After 8 or 32 hrs of incubation, $600 \mu \mathrm{L}$ of the media sample was collected from both cell lines and frozen at $70^{\circ} \mathrm{C}$ immediately. The frozen media was thawed prior to the assay. To remove cells in the media, the samples were centrifuged at $13,000 \mathrm{rmp}$ for $10 \mathrm{~min}$ and the supernatants were collected. The ELISA was performed as described below:

(1) uPA standards of $0.1 \sim 50 \mathrm{ng} / \mathrm{ml}$ was prepared in dilution buffer with BSA;

(2) The 96-well plate (8x12 removable strips) provided in the ELISA kit had already been coated with capture antibody, and blocked and dried. $100 \mu \mathrm{L}$ of the standard and unknown samples were then added to each well, in duplicates, and the plate was shaken at $300 \mathrm{rpm}$ for $30 \mathrm{~min}$;

(3) Blocking buffer (3\% BSA in TBS buffer) was used to dissolve primary antibody. $100 \mu \mathrm{L}$ of the primary antibody solution was added to each well and the plate was shaken at $300 \mathrm{rpm}$ for $30 \mathrm{~min}$;

(4) The wells were washed with $300 \mu \mathrm{L}$ washing buffer 3 times and removed. Remaining washing buffer was removed by gently tapping plate on paper towel;

(5) $2 \mu \mathrm{L}$ secondary antibody was diluted in $10 \mathrm{~mL}$ blocking buffer, and $100 \mu \mathrm{L}$ was added to each well. The plate was shaken at $300 \mathrm{rpm}$ for $30 \mathrm{mins}$ and washed with washing buffer 3 times;

(6) $100 \mu \mathrm{L}$ substrate was added to each well and the plate was shaken for 10 mins. The reaction was then stopped by adding $50 \mu \mathrm{L}$ of $1 \mathrm{~N} \mathrm{H}_{2} \mathrm{SO}_{4}$ stop solution to each well;

(7) The absorbance at $450 \mathrm{~nm}$ was immediately read by Spectra Gemini XPS fluorometer. The amount of uPA was quantified. 


\section{(b) Cell studies with GNP-SSP-Cy}

Cell study with GNP-SSP-Cy was performed at the Achilefu laboratory, Washington University, St. Louis, MO. MDA-MB-231 and MCF-7 cells were plated on Lab-Tek slides separately, at 3000 cells/well. The plates were incubated in serum-free DMEM with 100 units/mL Penicillin and 100 units/mL Streptomycin (for MCF-7 cells $0.01 \%$ Insulin is added), in $\mathrm{CO} 2$ incubator at $37^{\circ} \mathrm{C}$ overnight. The experiment with GNPSSP-Cy was done as follows:

(1) GNP-SSP-Cy was sonicated for 5 min to avoid any aggregation.

(2) GNP-SSP-Cy was added to the well containing the cells, at the Cypate concentration $10 \mu \mathrm{M}$, and incubated for $2 \mathrm{hrs}$.

(3) After incubation, the media in the well was pipetted out and put in a 96-well black plate, and the fluorescence was measured by a Pearl Imager.

\section{(c) Cell studies with GNP-LSP-mCy}

Cell study with GNP-LSP-Cy was performed at the Achilefu laboratory. The cell preparation was done the same as in (b). Then,

(1) GNP-LSP-mCy was sonicated for 5 min to avoid any aggregation; $\mathrm{mCy}$-BLK or GNP-LSP-mCy was added to the wells at the mCy concentration of $340 \mathrm{nM}$ (for GNP- $L$ SP-mCy the GNP concentration was $10 \mathrm{nM}$ );

(2) At 1, 3 and $24 \mathrm{hrs}$, the slides were removed from the wells and the cells were imaged by NIR fluorescence confocal microscope. 


\section{CHAPTER IV}

\section{RESULTS AND DISCUSSIONS}

\section{A. THEORETICAL ANALYSIS OF FLUORESCENCE ALTERATION BY GNP}

As described in the theory section (Chapter II), the fluorescence alteration by GNPs is affected by the GNP size, Ex/Em wavelengths and intrinsic quantum yield of the fluorophore, and the distance between the GNP and the fluorophore (Fig. 1). Once the fluorophore is selected, in a practical sense, the wavelengths of the Ex/Em light and the quantum yield are fixed. The GNP size and the distance between the fluorophore and the GNP are, therefore, the two parameters that can be manipulated. In this section, the effect of each parameter on the fluorescence alteration was analyzed for the fluorophores with various Ex/Em wavelengths $(490-830 \mathrm{~nm})$ and intrinsic quantum yields $(0.012-0.93)$, which are frequently used in biosensing/imaging applications. Table 1 in Chapter III lists the fluorophores (FITC, AF532, Cy3, AF647, AF700 and ICG) and their properties.

The mathematical model we are using is based on the interaction between a single fluorophore and a single GNP, with no inter-particle interaction (Chapter II, Part C, Theory). The system is symmetric in the azimuthal axis $(d E / d \phi=0)$ and the light is applied in the $z$ direction $(\theta=0)$. In our study, the following additional assumptions were made:

(1) The fluorophore is placed at $\theta=0$ (along the $z$ axis). 
(2) For each fluorophore, the excitation and emission are only at their peak wavelength values.

(3) The fluorophore and GNP are placed in water.

\section{Excitation Rate and System Parameters}

(a) GNP size and incident light wavelength

The GNP size for in vivo application is desired to be in the range of $10-100 \mathrm{~nm}$, because this range provides a long circulation time in blood and an effective cell-uptake rate [Davis, 2008]. The size includes the thickness of particle surface modification, such as coating of polymers and/or other biomolecules conjugated on the GNP surface. The core GNP size should be, therefore, smaller than the entire particle size. Based on these conditions, the maximum GNP core size studied was $50 \mathrm{~nm}$. For the distance between a fluorophore and a GNP (i.e., the spacer length), a range of 0-10 nm was chosen because this range provides a plasmon field strength sufficient for affecting the electron status of the fluorophore. It is also a range for a linear bio-spacer to maintain its length reasonably well. When a fluorophore is selected, the incident light wavelength to be used becomes the excitation peak wavelength of the fluorophore. Figure 4 shows the enhancement of the excitation decay rate (excitation rate) by a bare GNP of sizes 10,30 and $50 \mathrm{~nm}$ for the selected fluorophores at their respective excitation wavelengths, with the distance from the GNP surface.

For all three GNP sizes, on the GNP surface the excitation rates of a fluorophore are the same and they are the highest. The rates decrease rapidly with the distance and for smaller GNPs, the decrease is faster. Therefore, at the same distance from the GNP, the enhancement in excitation rate is greater for a bigger GNP. 

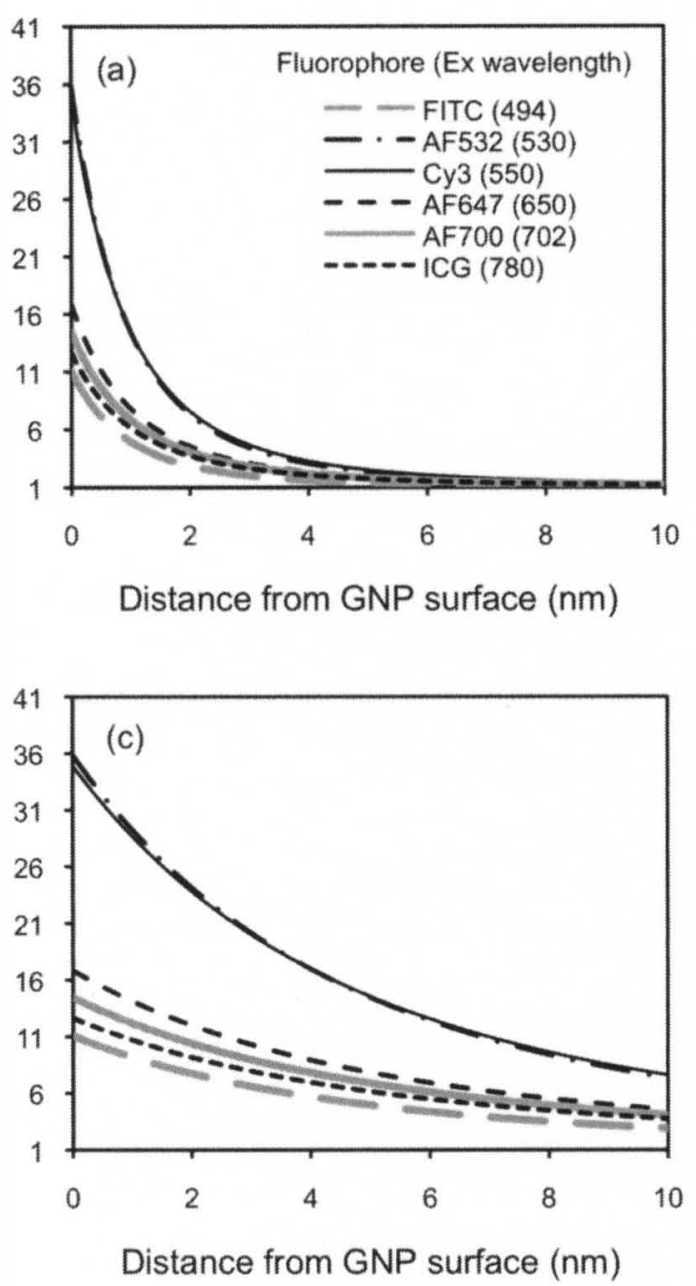

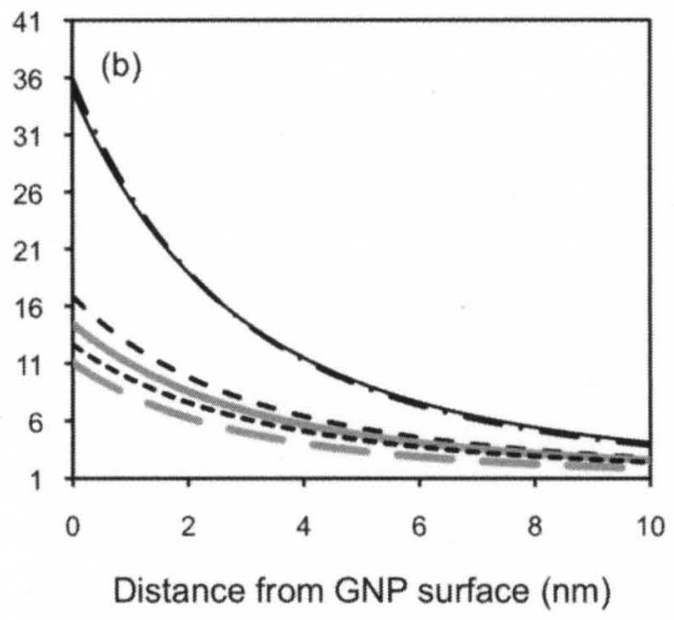

Figure 4. The enhancement in the excitation decay rates of fluorophores by their respective Ex wavelengths, with respect to the distance from the surface of a (a) $10 \mathrm{~nm}$ (b) $30 \mathrm{~nm}$ and (c) $50 \mathrm{~nm}$ GNP

In terms of the fluorophores (i.e., the wavelength of the incident light), AF532 (Ex $530 \mathrm{~nm}$ ) shows the highest enhancement (36 times on the surface). Its wavelength is slightly red-shifted from the plasmon resonance peak of GNP (520 nm). Hearting's analysis [2007] studied that, in a medium with a relative dielectric permittivity greater than 1 (The dielectric permittivity in our system is 1.76), the highest enhancement occurs at a wavelength slightly red-shifted from the resonance peak. The level for Cy3 (Ex 550 $\mathrm{nm}$ ) is only slightly lower. AF700 (Ex $702 \mathrm{~nm}$ ) and ICG (Ex $780 \mathrm{~nm}$ ) show lower 
enhancement (12 times on the surface). The lowest enhancement is for FITC, whose excitation peak is at $494 \mathrm{~nm}$, shorter than the GNP resonance wavelength.

\section{(b) Effect of coating material on GNP}

The plasmon field distribution is also affected by the materials coated on the GNP surface. For biomedical applications, GNPs are usually coated with a biocompatible and hydrophilic polymer to stabilize GNPs in water-based biological media. The coating also prevents protein adsorption on the GNPs. One of the most commonly used polymers is PEG. Figure 5 illustrates the plasmon field strength distributions, on and around a $10 \mathrm{~nm}$ GNP coated with PEG at a thickness of 1, 2 and $3 \mathrm{~nm}$, when the incident light at $780 \mathrm{~nm}$ is applied. $780 \mathrm{~nm}$ is the excitation wavelength peak of ICG, and it is used as an example because an ICG derivative is used in our experimental studies.

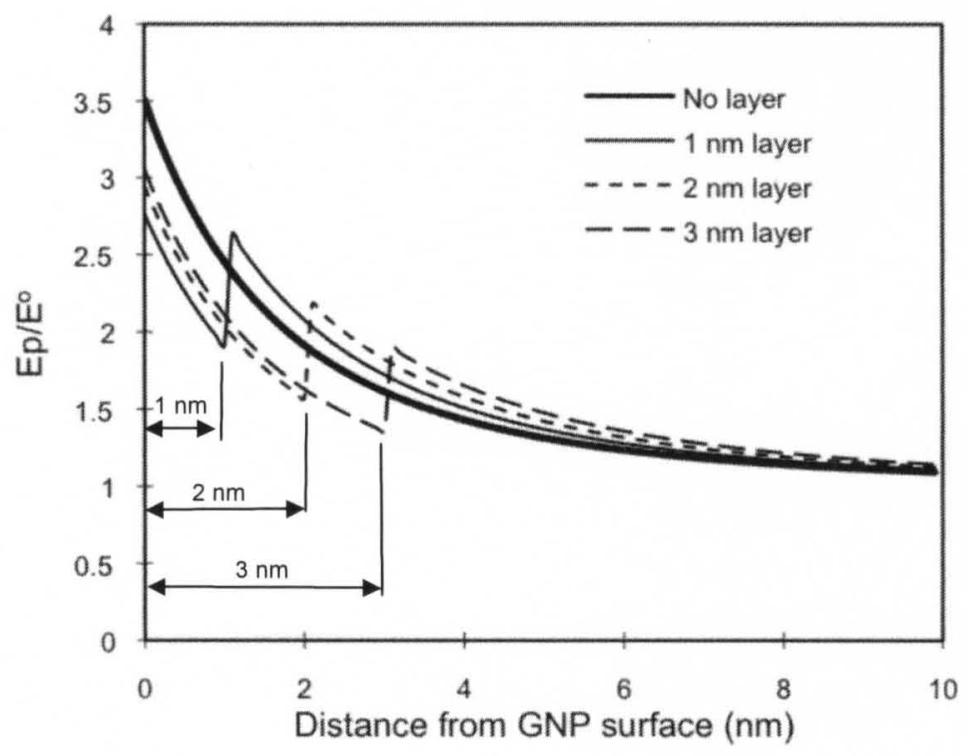

Figure 5. Plasmon field distributions computed on/around a $10 \mathrm{~nm}$ GNP coated with PEG, at a thickness of $0,1,2$, or $3 \mathrm{~nm}$, when the incident light at $780 \mathrm{~nm}$ is applied. 
For all three cases, the field strength inside the PEG layer is approximately $15 \%$ lower than that without the layer (Fig. 5). Therefore if the fluorophore is to be placed inside the polymer, then the reduction of the field strength should be considered. Immediately outside the layer, the field strength is slightly higher than that without the layer. As the distance increase, the strength becomes very close to the one without it. It should be noted that this trend is for the coating with dielectric materials. If the coating material is metal, the plasmon field may be even enhanced instead of being reduced.

\section{Quantum Yield Affected by Various Parameters}

The change in the quantum yield of a fluorophore by a GNP is the combined effect of the plasmon field strength on the radiative decay rate $\left(\gamma_{r}\right)$ and the absorption ( $\left.\gamma_{a h s}\right)$ of emitted fluorescence by GNPs, and the intrinsic quantum yield of the fluorophore, as shown in Eq. 15.

\section{(a) Radiative decay rate}

Since the radiative decay $\left(\gamma_{r} / \gamma_{r}^{a}\right)$ for the spherical particles may be assumed to be the same as $\gamma_{\text {ext }} / \gamma_{\text {exc }}^{o}$, it is higher for the bigger GNP and is the highest for the fluorophores with Ex wavelength slightly red-shifted from the GNP resonance peak.

\section{(b) Absorption}

The energy of the fluorophore absorbed by the GNP $\left(\gamma_{u t s}\right)$ is determined mainly by the Em wavelength of a fluorophore. Because the energy is absorbed by the GNPs, it is usually the highest at the resonance peak wavelength of GNPs. Figure 6 shows the absorption rates by a $30 \mathrm{~nm}$ GNP for the fluorophores with respect to the distance. The $\mathrm{y}$ - 
axis values are presented in a logarithmic scale due to the wide range of the number within the distance. For all fluorophores, on the GNP surface the absorption is extremely high $\left(10^{7}-10^{8}\right)$, and it decreases rapidly with the distance. FITC (Em $\left.521 \mathrm{~nm}\right)$ shows the highest absorption $\left(\sim 10^{8}\right)$, and remains very high over the distance studied. For the fluorophores with emission wavelength away from the resonance peak, the absorption rates are lower than that of FITC. For AF647, AF700 and ICG, the absorptions are the lowest.

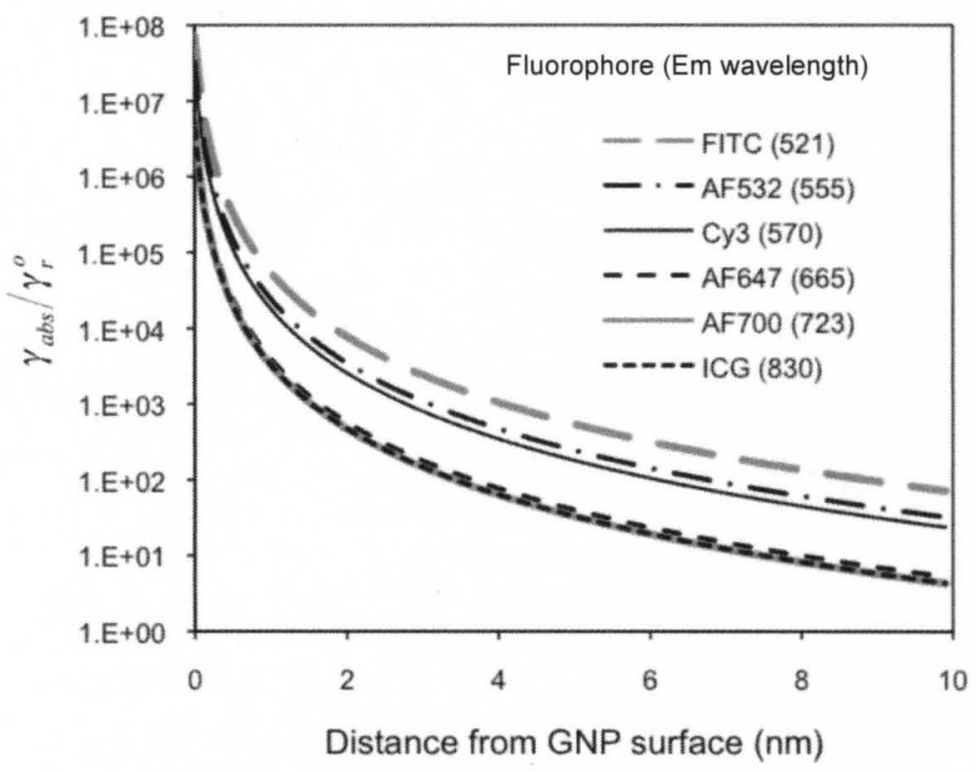

Figure 6. Absorption rate $\left(\gamma_{a b s} / \gamma_{r}^{o}\right)$ of various fluorophores by a $30 \mathrm{~nm}$ GNP, with respect to the distance from the GNP surface

\section{(c) Intrinsic quantum yield}

The effect of the intrinsic quantum yield $\left(q^{o}\right)$ on the fluorescence may be understood better when we write Eq. 3 in the following format:

$$
\frac{q}{q^{o}}=\frac{\gamma_{r} / \gamma_{r}^{o}}{q^{o}\left(\gamma_{r} / \gamma_{r}^{o}+\gamma_{a b s} / \gamma_{r}^{o}-1\right)+1}
$$


For the fluorophore with high intrinsic quantum yield $(\sim 1), q / q^{o}$ is mainly determined by the value of $1 /\left(1+\gamma_{a b s} / \gamma_{r}\right)$. For a fluorophore with very low $q^{o}(\sim 0.01)$ such as ICG, the value is larger. It also means that the fluorescence of those fluorophores have more tendency to be enhanced.

Table 3 summarizes values of the first and the second term of the denominator of Eq. 21 at $0.1 \mathrm{~nm}$ (close), $5 \mathrm{~nm}$ (middle) and $10 \mathrm{~nm}$ (far) from a $30 \mathrm{~nm}$ GNP surface and, the third term for the fluorophores.

Table 3. Normalized radiative decay rate and absorption rate at $0.1,5$ and $10 \mathrm{~nm}$ from a $30 \mathrm{~nm}$ GNP surface, and $\left(1-q^{\circ}\right) / q^{o}$ of the fluorophores.

\begin{tabular}{|c|c|c|c|c|c|c|c|}
\hline \multirow{2}{*}{ Fluorophores } & \multicolumn{2}{|c|}{$\begin{array}{c}0.1 \mathrm{~nm} \text { from GNP } \\
\text { surface }\end{array}$} & \multicolumn{2}{|c|}{$\begin{array}{c}5 \mathrm{~nm} \text { from GNP } \\
\text { surface }\end{array}$} & \multicolumn{2}{|c|}{$\begin{array}{c}10 \mathrm{~nm} \text { from GNP } \\
\text { surface }\end{array}$} & \multirow{2}{*}{$(1-q)^{o} / q^{\circ}$} \\
\hline & $\gamma_{r} /$ & $\gamma_{a b s} / \gamma_{r}^{n}$ & $\gamma_{r} / \gamma_{r}^{\prime \prime}$ & $\gamma_{a b s} \gamma_{r}^{\prime \prime}$ & $\gamma_{r} \gamma_{r}^{n}$ & $\gamma_{a b s} \gamma_{r}^{a}$ & \\
\hline FITC & 10.1 & $9.0 \times 10^{6}$ & 3.4 & 543.6 & 1.9 & 72.1 & 0.1 \\
\hline AF532 & 32.4 & $4.1 \times 10^{6}$ & 9.2 & 244.5 & 3.9 & 32.4 & 0.6 \\
\hline $\mathrm{Cy} 3$ & 31.6 & $3.0 \times 10^{6}$ & 9.4 & 180.0 & 4.1 & 23.9 & 5.7 \\
\hline AF647 & 15.4 & $0.66 \times 10^{6}$ & 5.4 & 40.0 & 2.8 & 5.3 & 2 \\
\hline AF700 & 13.2 & $0.54 \times 10^{6}$ & 4.8 & 32.7 & 2.6 & 4.3 & 3 \\
\hline ICG & 11.6 & $0.55 \times 10^{6}$ & 4.4 & 33.0 & 2.4 & 4.4 & 82.3 \\
\hline
\end{tabular}

When these three terms are compared, near the surface of the GNP $(0.1 \mathrm{~nm})$, the absorption term $\gamma_{\text {abs }} \gamma_{r}^{\circ}$ dominates for all the fluorophores. As the distance from the GNP increases, both $\gamma_{r} / \gamma_{r}^{\prime}$ and $\gamma_{a b s} / \gamma_{r}^{\prime \prime}$ decrease, while $\gamma_{\text {ats }} / \gamma_{r}^{\prime \prime}$ decreases much faster. At $5 \mathrm{~nm}$ from the GNP surface, the $\gamma_{\text {ats }} \gamma_{r}^{\prime \prime}$ values for the fluorophores with the emission wavelengths close to the GNP resonance peak (FITC, AF532, and Cy3) are still much higher than $\gamma_{r} / \gamma_{r}^{\prime \prime}$ or $\left(1-q^{\prime \prime}\right) / q^{\prime \prime}$. Therefore, the fluorescence tends to be still quenched. For the fluorophores whose Em peaks are away from the GNP resonance wavelength 
(AF647, AF700, and ICG), at $5 \mathrm{~nm}$, their $\gamma_{a b s} / \gamma_{r}^{o}$ values decrease to the magnitude comparable to $\gamma_{r} / \gamma_{r}^{o}$ and $\left(1-q^{o}\right) / q^{o}$, and their quenching effect dominates no longer. At $10 \mathrm{~nm}, \gamma_{r} / \gamma_{r}^{o}$ decreases up to $1 / 3 \sim 1 / 2$ of the value at $5 \mathrm{~nm}$ away, while $\gamma_{\text {abs }} / \gamma_{r}^{o}$ decreases to $\sim 1 / 8$ of its value at $5 \mathrm{~nm}$.

Figure 7a illustrates the quantum yield $\left(q / q^{o}\right)$ for the fluorophores near a $30 \mathrm{~nm}$ GNP. ICG is discussed separately (Fig. 7b) because it has an unusually low quantum yield (0.012). For all fluorophore, the $q / q^{o}$ values are the lowest at the GNP surface and increase with the distance. For FITC, AF532 and Cy3, the values are extremely low even at $10 \mathrm{~nm}$ away from GNP surface due to their large $\gamma_{a b s} / \gamma_{r}^{o}$ values. AF700 has greater $q / q^{o}$ values than other fluorophores because of its smaller $\gamma_{a b s} / \gamma_{r}^{o}$ and $q^{o}$ values. When it is $10 \mathrm{~nm}$ away from the GNP surface $q / q^{o}$ becomes greater than 1 .
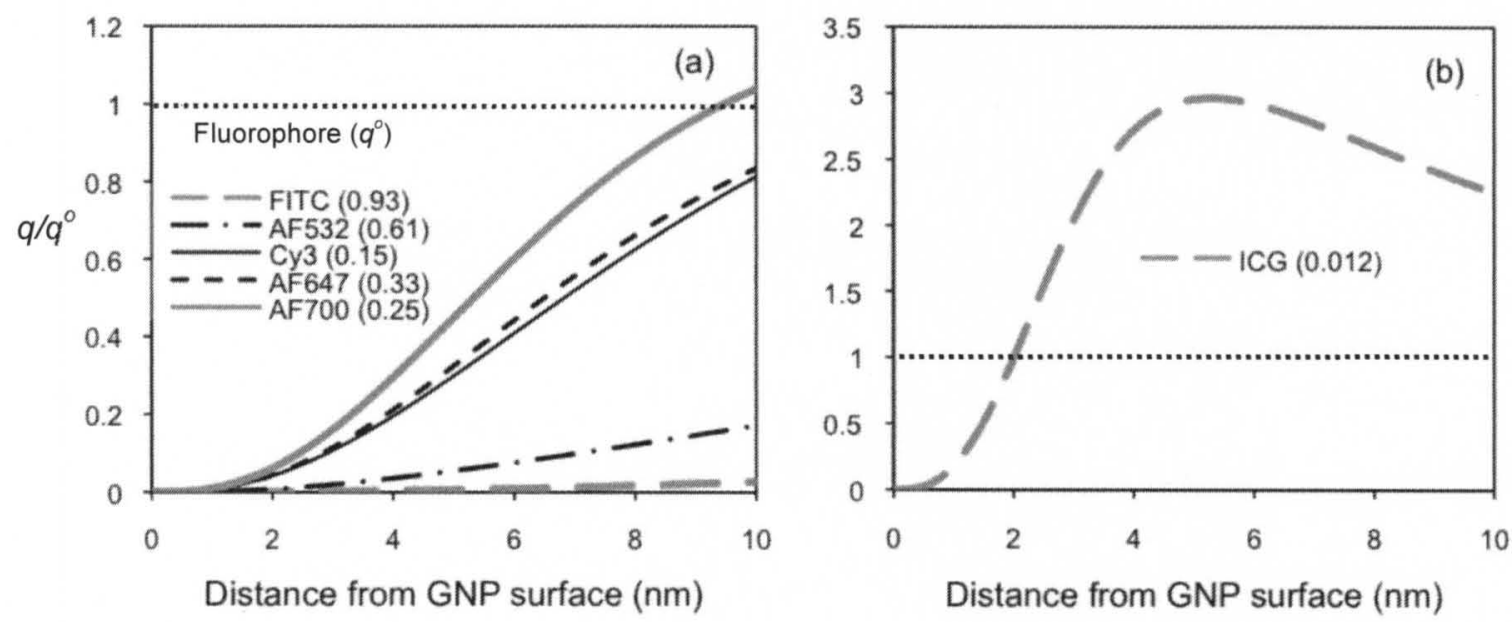

Figure 7. Theoretically computed quantum yield of (a) various fluorophores (b) ICG with respect to the distance from a $30 \mathrm{~nm}$ GNP

For ICG (Fig. 7b), the resulting quantum yield with respect to the distance from the GNP shows a unique profile. At the distance less than $1 \mathrm{~nm}$, the $q / q^{\circ}$ is very low. It 
becomes greater than 1 when the distance is greater than $2 \mathrm{~nm}$. It reaches the greatest $(3.4$ times) at $4.2 \mathrm{~nm}$, and then slowly goes down toward 1 . Therefore within the distance of 5 $\mathrm{nm}$, the $q / q^{o}$ value changes from the minimum (on the surface) to the maximum (at 4.2 $\mathrm{nm})$.

\section{Resulting Fluorescence Output}

The fluorescence output affected by a GNP is the combination of the enhancement in excitation decay rate and the change in quantum yield (Eq. 9). Figure 8 shows the fluorescence output of the fluorophores, by a (a) $10 \mathrm{~nm}$, (b) $30 \mathrm{~nm}$, and (c) 50 nm GNP. Among the fluorophores studied, ICG shows a unique response to the plasmon field generated by the GNP due to its NIR Ex/Em wavelengths and extremely low intrinsic quantum yield. Therefore, the fluorescence output for ICG will be analyzed separately. For all fluorophores, strong quenching occurs at the GNP surface. FITC shows almost complete quenching ( $>99 \%)$ throughout the distance studied, for all three GNP sizes. It is mainly because its emission wavelength $(521 \mathrm{~nm})$ is close to the plasmon resonance peak of GNP and the energy absorption by GNP is very high. FITC is, therefore, a good candidate for the fluorescence quenching, but not for the enhancement.

With a $10 \mathrm{~nm}$ GNP (Fig. 8a), for all fluorophores, the fluorescence is quenched within $10 \mathrm{~nm}$ from the GNP surface. Therefore, $10 \mathrm{~nm}$ GNP may be a good candidate for conditional fluorescing, such as molecular beacon, for these fluorophores.

As the GNP size increases, the level of fluorescence becomes higher for all fluorophores. For a $30 \mathrm{~nm}$ GNP, the fluorescence levels of Cy3, AF647 and AF700 are quenched within $2 \mathrm{~nm}$ from the GNP surface, and start to get enhanced when the distance 
is greater than $3 \mathrm{~nm}$. When the distance becomes approximately $9 \mathrm{~nm}$, the enhancement becomes 2.5-4 times and afterwards, slowly decreases.

For a $50 \mathrm{~nm}$ GNP, the distance for fluorescence quenching is even shorter and the fluorescence enhancement is greater and starts at a shorter distance than for the $30 \mathrm{~nm}$ GNP. The enhancement for Cy3, AF647 and AF700 is up to 5-10 times. Even the fluorescence of AF532 gets enhanced at a distance of $4.5 \mathrm{~nm}$. The distance for the maximal enhancement is longer for the larger GNPs.
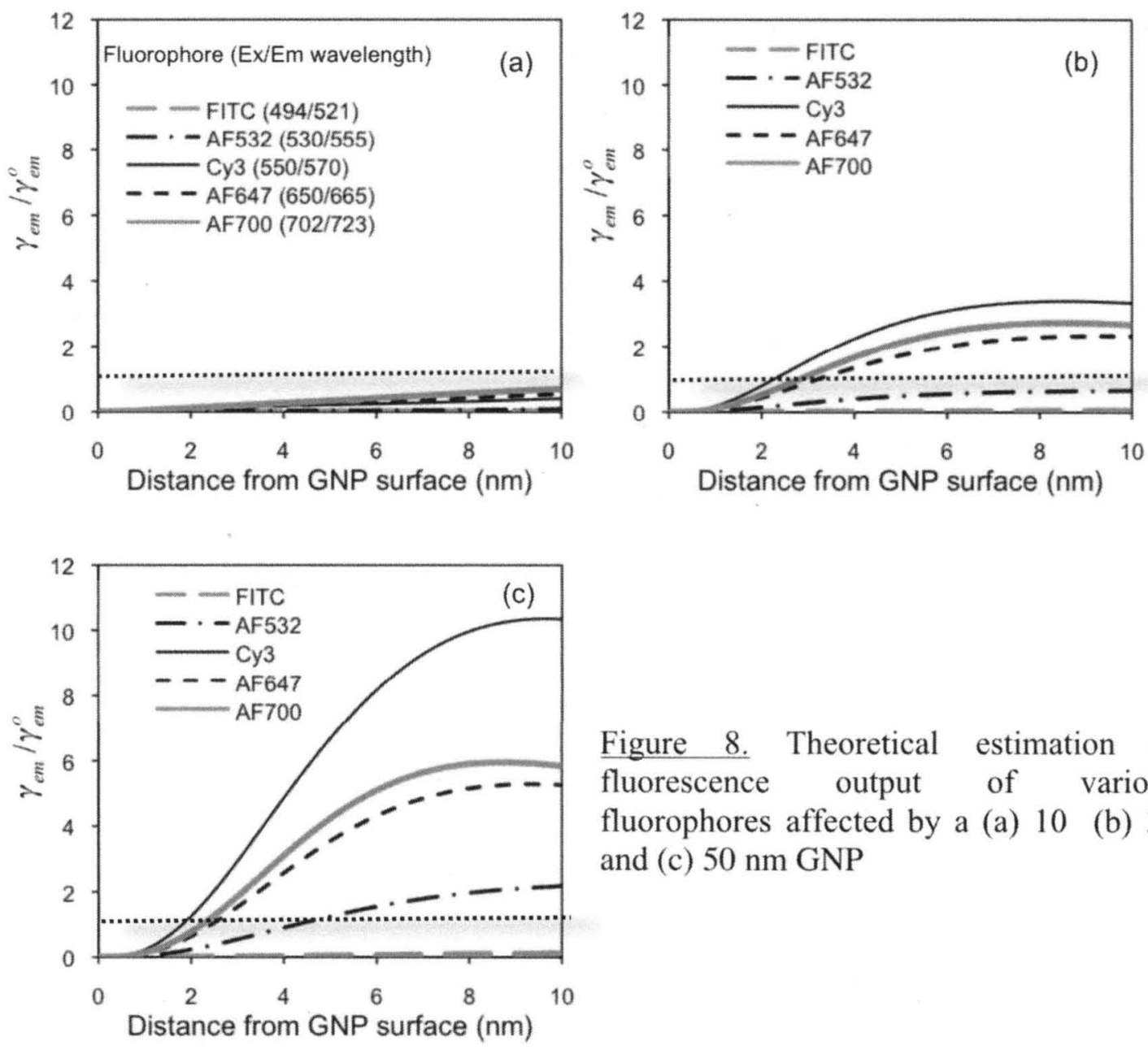

Figure 8. Theoretical estimation of fluorescence output of various fluorophores affected by a (a) 10 (b) 30 and (c) $50 \mathrm{~nm}$ GNP 
The theoretical study results for fluorescence manipulation using GNPs may be summarized as follows:

\section{For fluorescence quenching:}

(1) The fluorophores with the Em wavelength near the GNP plasmon resonance peak are good candidates.

(2) On the GNP surface, the fluorescence of fluorophores is always quenched.

(3) For the fluorophores with Em wavelengths significantly longer than the GNP resonance peak, e.g., far-red or NIR fluorophores, the distance between the GNP and the fluorophore needs to be shorter to achieve the same level of quenching.

(4) Bigger GNPs require shorter distance from the fluorophore to quench the fluorescence.

\section{For fluorescence enhancement:}

(1) Only the fluorophores with $\mathrm{Ex} / \mathrm{Em}$ wavelengths longer than the resonance peak wavelength of GNP, may be effectively used for fluorescence enhancement.

(2) The GNP size needs to be sufficiently large to generate a plasmon field strong enough to enhance fluorescence. In general, bigger GNPs provide greater fluorescence enhancement.

(3) The fluorophores with a lower quantum yield have more potential for the fluorescence enhancement.

(4) To obtain the maximal fluorescence enhancement, the distance between a fluorophore and a GNP needs to be accurately selected. 


\section{ICG Fluorescence Alteration by GNPs}

The fluorescence of ICG altered by a GNP was studied more in details here because ICG is the basis of Cypate, the fluorophore used in our experimental studies, and because it has Ex/Em wavelength in NIR and a very low quantum yield (Table 1).

Figure 9 shows the enhancement of the excitation decay rate by ICG excitation wavelength $(780 \mathrm{~nm})$ by GNPs at the size range of our interest, with the distance from the GNP surface. As for other fluorophores, larger GNPs provide greater enhancement, although the values are lower $(\sim 12)$ than those for the fluorophores close to the GNP resonance wavelengths $(\sim 36)$.

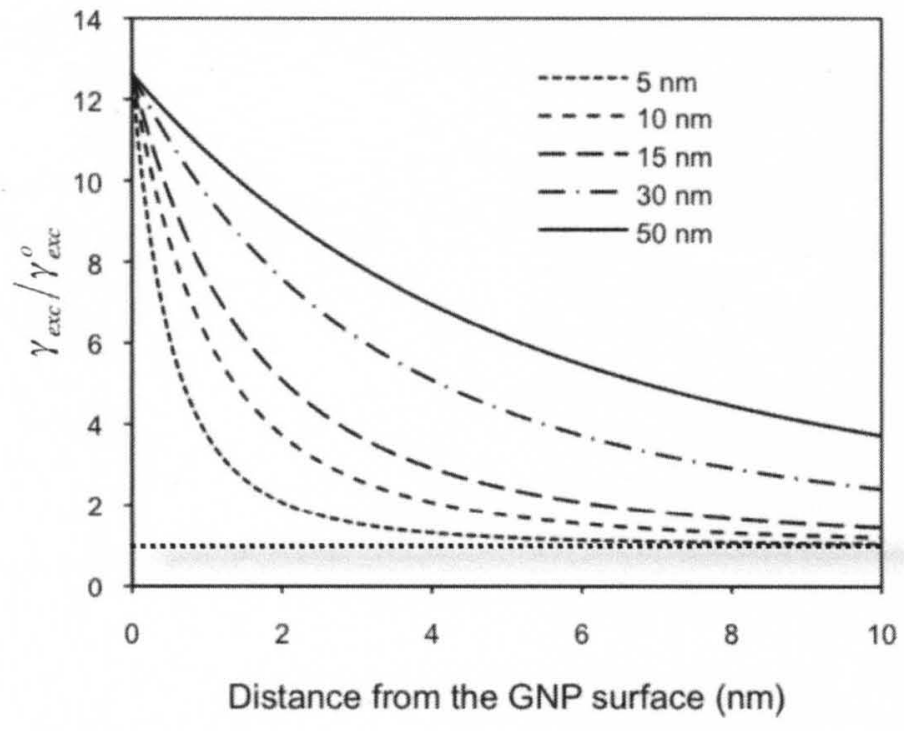

Figure 9. The enhancement in the excitation decay rates of ICG (Ex wavelength $780 \mathrm{~nm}$ ) by GNPs at various sizes.

For the quantum yield of ICG, the effects of the absorption rate and the intrinsic quantum yield were analyzed in detail because of the uniqueness of ICG in these two aspects. The absorption rate $\left(\gamma_{a b s} / \gamma_{r}^{o}\right)$ of ICG by GNP with distance from GNP is shown in Figure 10. Previously the values for other fluorophores were presented in logarithmic 
scale (Fig. 6), but to provide a better view of the values for ICG in the distance and to compared it to the excitation rate, here, the values are presented in a linear scale. The absorption rate on the GNP surface is extremely high $\left(\sim 10^{6}\right)$, but the rate decreases very fast with distance. Fig. 10b shows the magnified section of the absorption rate in the distance range of 2-10 $\mathrm{nm}$. At $2 \mathrm{~nm}$, it already decreases to 470 . The absorption still decreases fast after $2 \mathrm{~nm}$. At $5 \mathrm{~nm}$, the absorption decrease to 33, and at $10 \mathrm{~nm}, 4.4$, a similar magnitude as the radiative decay rate and smaller than the intrinsic quantum yield effect $\left(1-q^{o}\right) / q^{o}$ (Table 3).

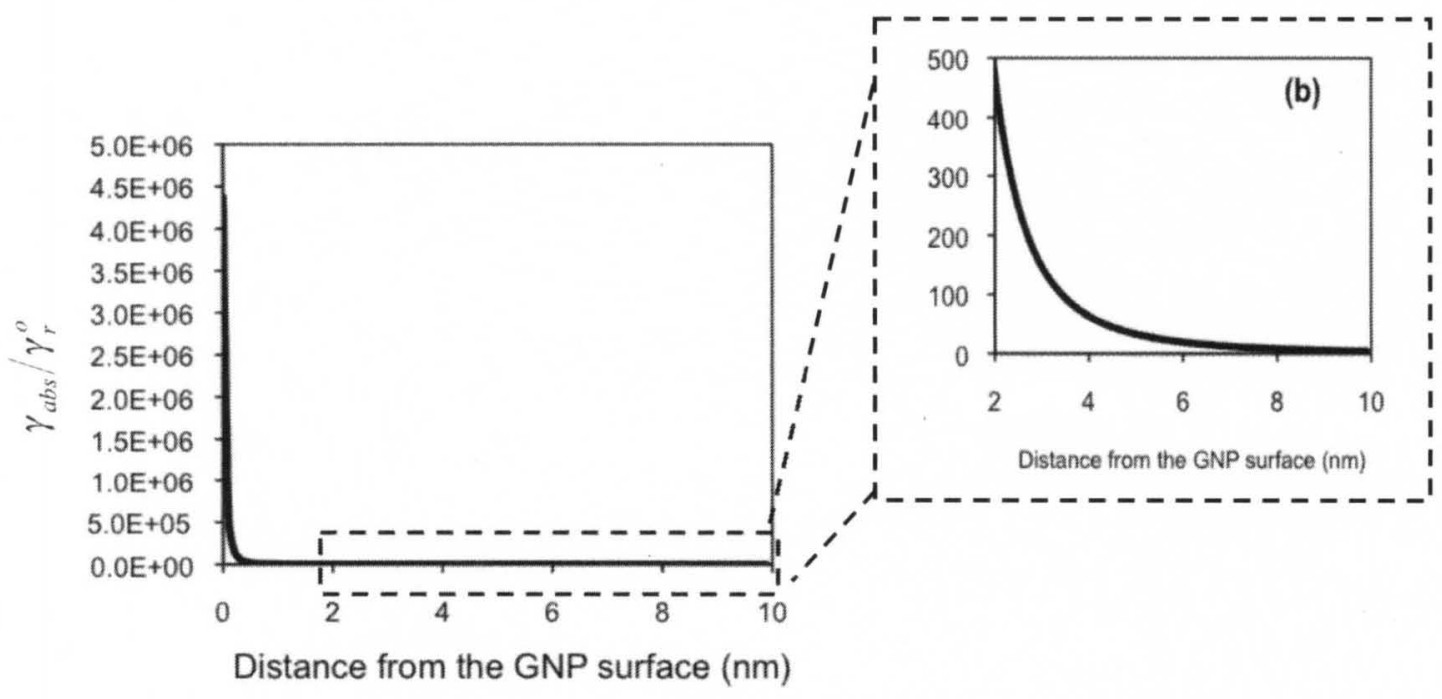

Figure 10. (a) Absorption rate $\left(\gamma_{a b s} / \gamma_{r}^{o}\right)$ of ICG by a $30 \mathrm{~nm}$ GNP, with respect to the distance from the GNP surface (b) magnified view of the section in the rectangle in (a)

Figure 11 shows the quantum yield change of ICG near the GNP at various sizes, which is the combined effect of the in radiative decay rate, absorption rate and intrinsic quantum yield. Bigger GNPs provide greater quantum yield. When the distance is less than $1 \mathrm{~nm}$, the quantum yield is highly reduced due to the strong absorption shown in Fig. 10a. As the distance increase, the absorption decreases much faster than the radiative 
decay rate, and the quantum yield increases until it reaches the maximum, and decreases thereafter.

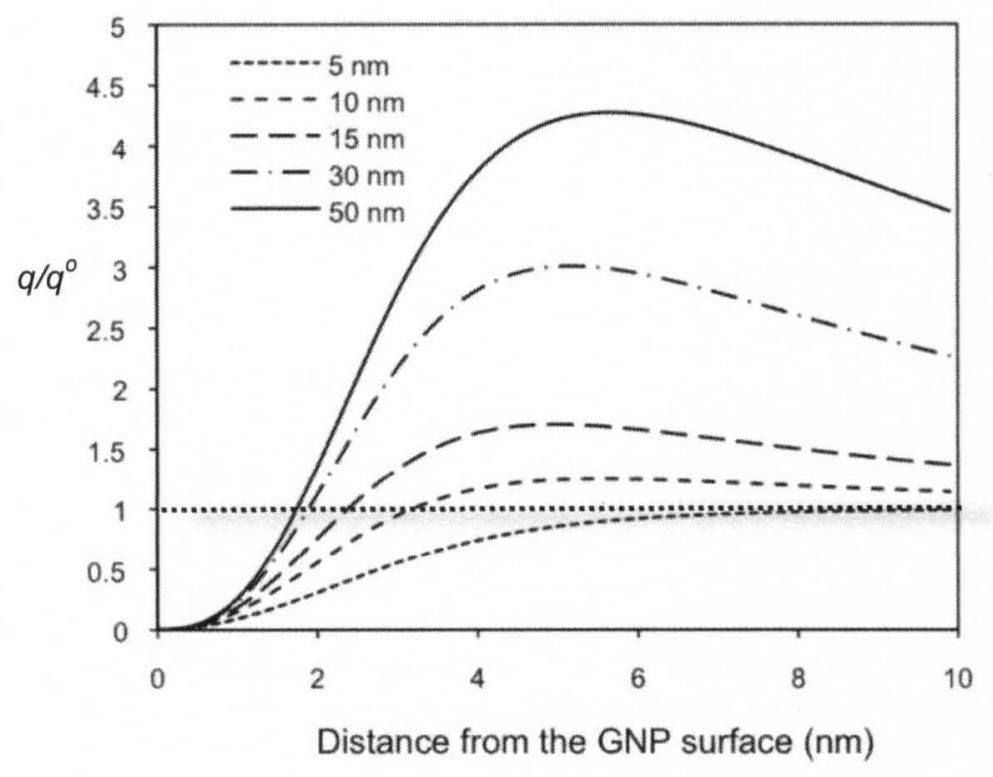

Figure 11. Theoretically computed quantum yield change of ICG (Ex/Em $780 / 830 \mathrm{~nm}$ ) with the distance from the GNP at various sizes

The fluorescence output is the product of the excitation rate and the quantum yield (Eq. 9; Figs 9 and 10). Figure 12 shows the fluorescence change of ICG by a GNP of the size $5 \sim 50 \mathrm{~nm}$. With $5 \mathrm{~nm}$ GNP, the ICG fluorescence is quenched within $4 \mathrm{~nm}$. At the distance longer than $4 \mathrm{~nm}$, the level is similar to that without the GNP. Therefore, 5 nm GNP may be effectively used as a quencher for ICG. For the $10 \mathrm{~nm}$ GNP, the fluorescence is quenched only within $1.3 \mathrm{~nm}$ from the GNP, and gets enhanced thereafter. It reaches the highest enhancement (2.4 times) at $3.4 \mathrm{~nm}$. As the GNP size increase, the distance for quenching becomes even shorter, and the maximal fluorescence enhancement becomes higher. In general, the distance for the maximal enhancement is shorter than those for other fluorophores. For bigger GNPs, the distance for maximal 
enhancement is longer, as for other fluorophores. For the 15, 30 and $50 \mathrm{~nm} \mathrm{GNPs,} \mathrm{the}$ maximal enhancements are 5, 14, and 26 times, at the distance of 3.4, 3.9 and $4.4 \mathrm{~nm}$, respectively. The enhancement values are much greater than those for the other fluorophores studied, mainly due to its very low intrinsic quantum yield.

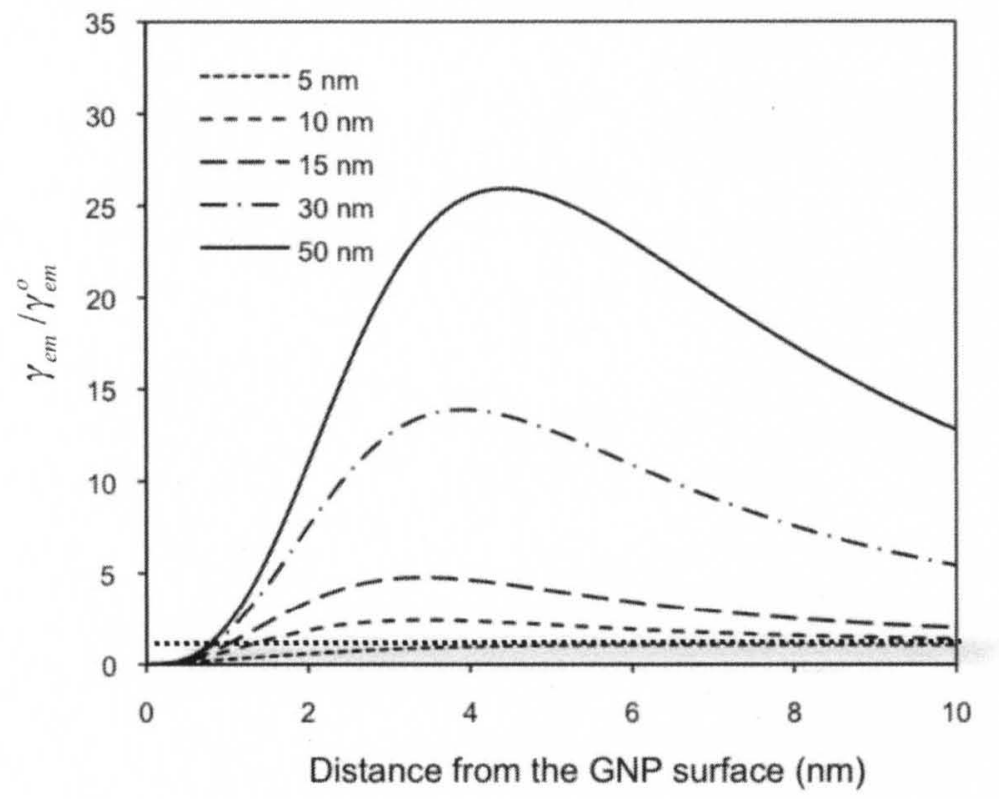

Figure 12. Theoretically computed fluorescence emission of ICG (Ex/Em $780 / 830 \mathrm{~nm}$ ) with the distance from the GNP of various sizes

As can be seen from Figs. 8-12, the fluorescence alteration of ICG features a unique pattern. Both quenching and the maximal enhancement occur within a short $(\sim 5$ $\mathrm{nm}$ ) distance range. This unique property became the basis for our design of the contrast agent that conditionally changes fluorescence level from quenching to enhancement at presence of a particular enzyme, which is presented in the experimental section of this dissertation. 


\section{B. EXPERIMENTAL STUDY}

ICG fluorescence is predicted to be changed by GNP from complete quenching to extensive enhancement, within $5 \mathrm{~nm}$ from the GNP surface as shown in the theory section (Fig. 12). Utilizing this unique property, a novel optical contrast agent for molecular imaging was designed (Fig. 13). As a model system, the contrast agent was developed for breast cancer detection.

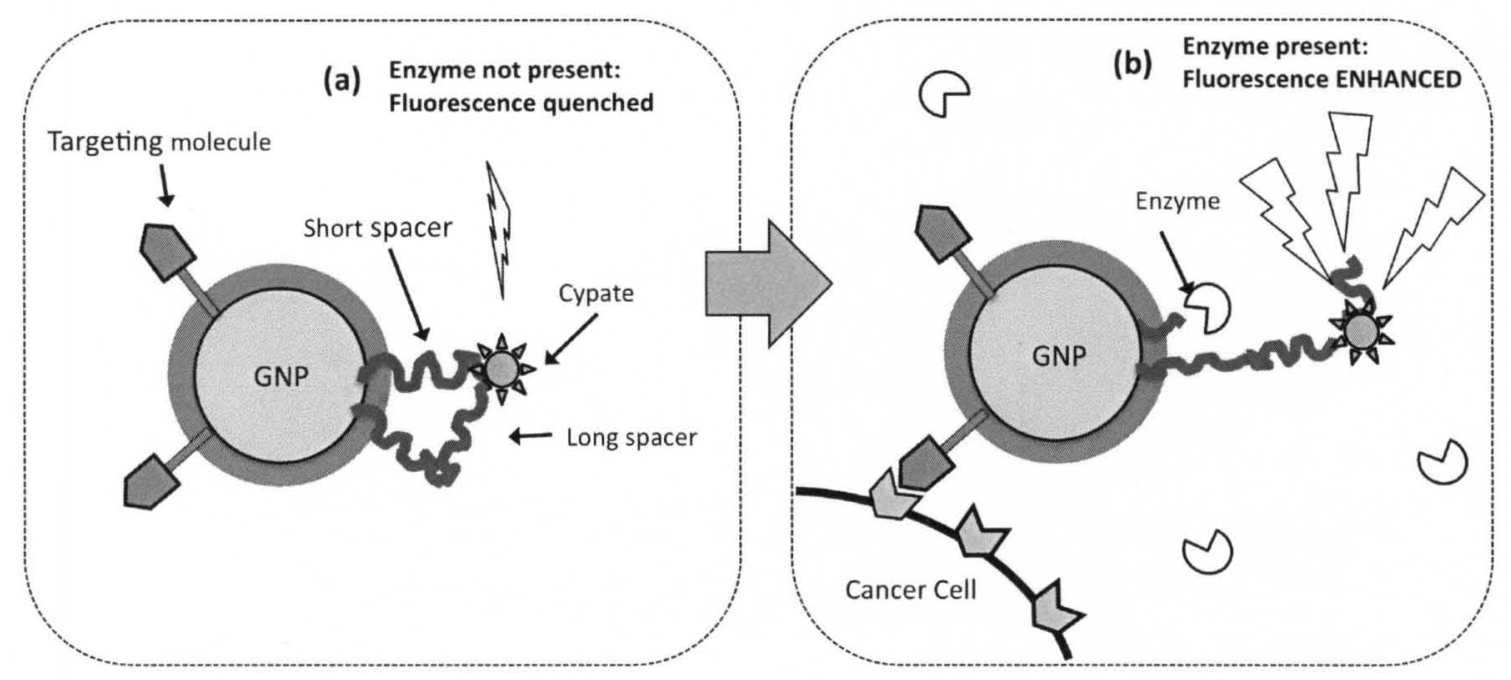

Figure 13. (a) Design of highly cancer specific fluorescing cancer locator. The agent normally does not emit fluorescence because the short spacer place Cypate very close to the GNP. (b) When the cancer locater binds to the cancer cell, it is exposed to the cancer secreting enzyme. The short spacer is cleaved by the enzyme from cancer cell and the fluorescence is emitted at an enhanced level.

\section{Components of the Contrast Agent}

The components of the contrast agent are: a GNP, the materials for GNP surface treatment (sPEG), spacers (SSP and LSP), Cypate and targeting molecules. 


\section{(a) GNP}

In our experimental studies, the GNP core at the sizes of 5, 10 and $15 \mathrm{~nm}$ were used. It should be noted that the sizes 5, 10 and 15 were the ones claimed by the manufacturer. The actual sizes according to our measurements by the light scattering analysis and TEM were $3.7,8$ and $16.4 \mathrm{~nm}$, respectively.

\section{(b) GNP surface modification}

The SAM of HS- $\left(\mathrm{CH}_{2}\right)_{11}-\left(\mathrm{CH}_{2} \mathrm{CH}_{2} \mathrm{O}\right)_{3}$ (sPEG) was used to stabilize GNPs. The HS- group is for reacting to GNP surface. The hydrocarbon chain section $(\sim 1.5 \mathrm{~nm}$ by the molecular simulation) of sPEG is straight and hydrophobic for tight packing on the GNP surface, and the PEG section ( $0.9 \mathrm{~nm}$ by the simulation) is flexible and hydrophilic. The length estimation was done by a computer code assuming that the molecule was in an extended configuation in vacuum, and, therefore, the actual length may be slightly different in the physiological conditions.

\section{(c) Spacers}

\section{(c-i) Short spacer (SSP)}

A short spacer was used to place Cypate at a distance from the GNP for its fluorescence to be quenched. The spacer must contain a motif that can be cleaved by a cancer-secreting enzyme. In our model system, the enzyme was urokinase-type plasminogen activator ( $\mathrm{UPA}$ ). $\mathrm{uPA}$ is expressed in a number of malignant breast cancers. The Gly-Gly-Arg amino sequence is a substrate of uPA, and, therefore, our short spacer was HS- $\left(\mathrm{CH}_{2}\right)_{2}$-Gly-Gly-Arg-Gly-Gly-Gly- $\mathrm{NH}_{2}$ (or $-\mathrm{ONH}_{2}$ ). The HS- ending is for 
binding it to the GNP surface and the $-\mathrm{NH}_{2}$ or $-\mathrm{ONH}_{2}$ ending is to link it to Cypate or mCypate.

\section{(c-ii) Long spacer (LSP)}

The long spacer is to keep the distance between Cypate and the GNP at the length producing the maximum fluorescence enhancement. The spacers were designed to have a similar structure as the sPEG to allow the LSP forming a well-mixed monolayer with sPEG on the GNP surface. They were $\mathrm{HS}-\left(\mathrm{CH}_{2}\right)_{\mathrm{m}}-\mathrm{PEG}_{\mathrm{n}}-\mathrm{ONH}_{2}$. By varying the numbers $m$ (12 or 16$)$ and $n(4,6$ or 8$)$, the length of the spacer was varied. The $-\mathrm{ONH}_{2}$ ending is for m-Cypate conjugation.

\section{(d) Fluorophore}

Our fluorophore is Cypate, an ICG derivative. ICG does not have any functional group to conjugate biomolecules (Figure 14a). Achilefu, et al. [2000; 2002] slightly modified the structure of ICG and created Cypate with two carboxylic groups (Figure 14b), which can then react to amine groups of biomolecules by using a coupling agent to activate the carboxylic groups. However, there are many biomolecules with carboxylic or amine groups. Therefore, we decided to use $-\mathrm{CHO} /-\mathrm{ONH}_{2}$ reaction. The Nantz-group, our research partner, added adelhyde (-CHO) groups to the carboxylic ends of Cypate to form a modified-Cypate (m-Cypate; Figure 14c). The adelhyde group allows the oxime coupling with the aminooxy ending $\left(-\mathrm{ONH}_{2}\right)$, forming stable oxime bound. This reaction does not require additional coupling agent and is not interfered by other peptides and proteins. 
If only one of the two-CHO endings of m-Cypate needs to be reacted, blocking agent (BLK), $\left(\mathrm{CH}_{3}\right)_{3} \mathrm{~N}^{+}-\left(\mathrm{CH}_{2}\right)_{2}-\mathrm{ONH}_{2}$, was used to block one ending. The BLK also helps to increase the hydrophilicity of Cypate, preventing from m-Cypate being adsorbed to the hydrophobic hydrocarbon chain of the SPEG or LSP.

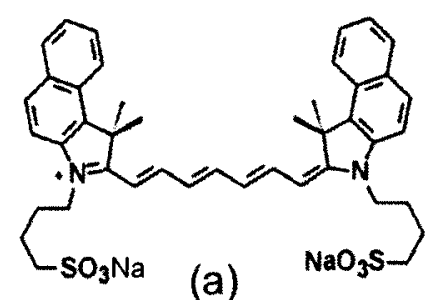

(a)

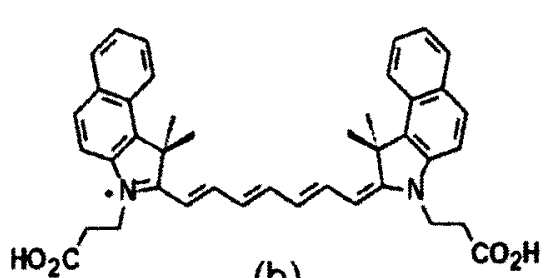

(b)

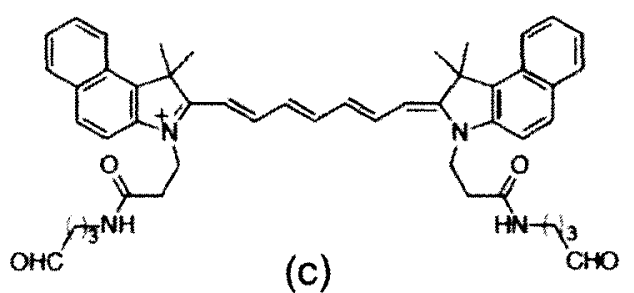

Figure 14. Molecular structures of (a) ICG and (b) Cypate (Supplied by the Achilefu group ) (c) m-Cypate (Modified by the Nantz group)

\section{(e) Targeting molecule}

Tamoxifen and Herceptin are selected to be the targeting molecules. Tamoxifen is a drug currently used to treat estrogen receptor positive $(E R+)$ breast cancer. It works as an antagonist that binds to the ER, which is over-expressed in $70 \%$ of breast cancer. Herceptin is an antibody that binds to Human epidermal growth factor receptor 2 (HER2), which is over-expressed in $20-30 \%$ of early-stage breast cancer.

This contrast agent is to emit little fluorescence (the fluorescence is to be quenched). It is to be preferentially delivered to the cancer site via the targeting molecule (Fig. 10b). The the agent is placed in an environment with uPA. When the short spacer is 
cleaved by uPA, the distance between the Cypate and the GNP is now at the long spacer length. The fluorescence changes from a quenched level to an enhanced level, detecting the cancer with high specificity and contrast.

The development steps of this contrast agent were as follows:

1. Characterization of individual components of the system

2. Optimization of spacers

(a) Optimization of the short spacer (conditional fluorescence quenching)

(b) Optimization of the long spacer (maximal fluorescence enhancement)

(c) Construction and testing the dual-spacer

3. Conjugation of targeting molecule

4. Cell study

In this dissertation the results from the steps 1,2, and a part of 4 are presented.

\section{Characterization of the Components}

\section{(a) The effect of the solvent on Cypate fluorescence}

Cypate is hydrophobic due to its aromatic part and long polymethine chain. It has a low solubility in water and forms aggregates in water [Licha, et al., 2000]. The intrinsic quantum yield (QY) of Cypate in water (0.0028) is lower than that in blood plasma (0.012). One way of avoiding the Cypate aggregation in water is by increasing the ionic strength in water. Figure 15 shows the fluorescence of Cypate in water, and $10 \mathrm{mM}$ PBS. The Cypate fluorescence is extremely low in water. In PBS, the Cypate fluorescence is approximately 150 times higher than that in water. The fluorescence in $1 \mathrm{mM}$ PBS is similar to that in $10 \mathrm{mM}$ PBS (only 7\% less). Colloidal GNPs stabilized with citric/tannic acids, without special surface treatment, easily precipitate in the solutions with high ionic 
strength (e.g., $10 \mathrm{mM}$ PBS buffer). Therefore, in the preliminary studies involving colloidal GNPs, 1 mM PBS buffer was used.

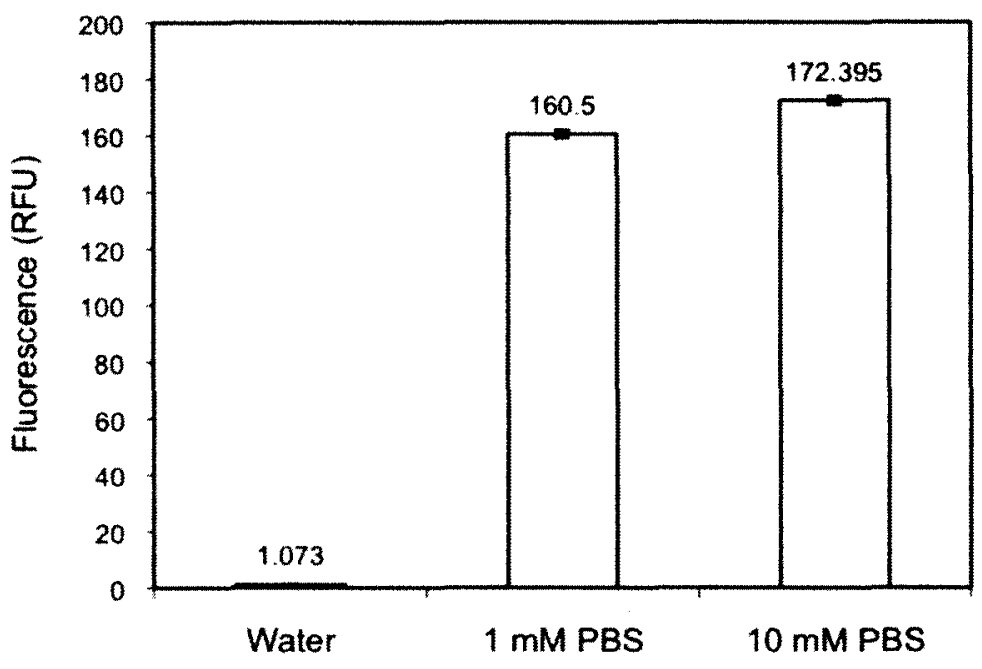

Figure 15. Fluorescence of Cypate in water, 1 and $10 \mathrm{mM}$ PBS buffer (Cypate concentration: $30 \mu \mathrm{M}$ )

\section{(b) Cypate concentration and self-quenching}

Fluorophores quench their own fluorescence when their concentration exceeds a certain level (inter-molecular quenching), either by the collision among excited and ground-stated fluorophores (dynamic quenching) or by forming the ground-stated fluorophore complexes (static quenching) [Ingle and Crouch, 1988; Johansson, et al., 2002]. To avoid the inter-molecular quenching, we have observed the Cypate fluorescence level with respect to the concentration at a range of $5 \sim 150 \mu \mathrm{M}$ (Fig. 16). 


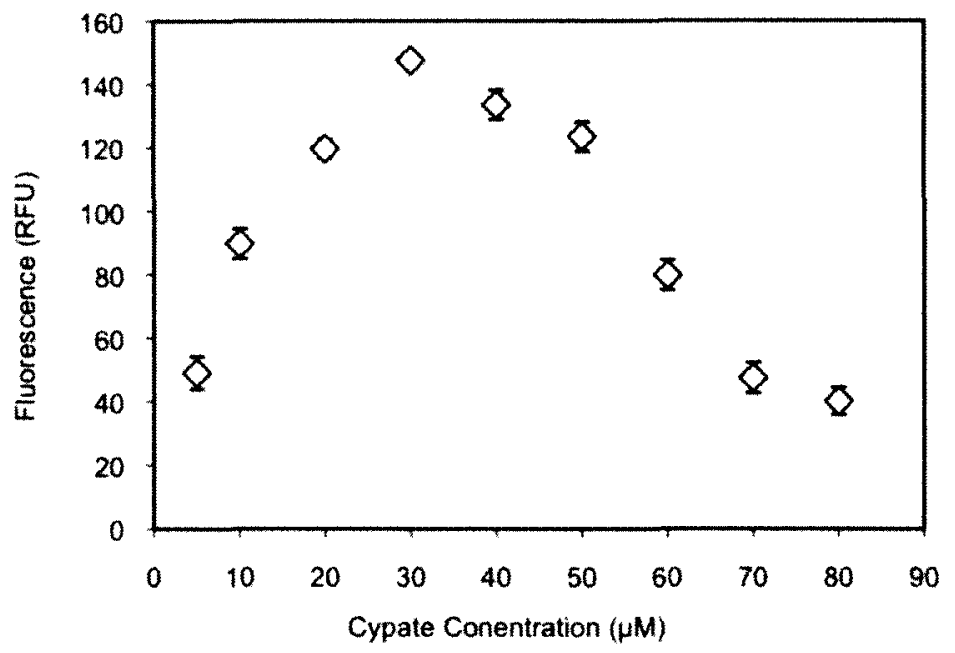

Figure 16. Fluorescence of Cypate at various Cypate concentrations (in 10 mM PBS)

The fluorescence increased with the increase in Cypate concentration up to 30 $\mu \mathrm{M}$. At concentrations higher than $30 \mu \mathrm{M}$, the fluorescence decreased. Therefore, the Cypate concentration less than $30 \mu \mathrm{M}$ was used for further studies.

\section{(c) Fluorescence of free Cypate affected by free GNP colloids}

One simple way of changing the average distance between the GNP and the Cypate is by varying the concentration of GNPs. The fluorescence of free Cypate affected by colloidal GNPs of various concentrations was studied for 5 and $10 \mathrm{~nm}$ GNPs at a weight (wt) concentration range of $0 \sim 60 \mu \mathrm{g} / \mathrm{ml}$ (molar concentration: for $5 \mathrm{~nm} \mathrm{GNP}$, $0 \sim 75 \mathrm{nM}$, and for $10 \mathrm{~nm} \mathrm{GNP}, 0 \sim 8.5 \mathrm{nM}$ ) at a Cypate concentration $30 \mu \mathrm{M}$, which is the maximum concentration without self-quenching (Fig. 17). In this experiment, GNP concentration is, therefore, the limiting factor.

When the GNP concentration is very low $(<6 \mu \mathrm{g} / \mathrm{ml})$, the GNPs enhanced the fluorescence. This phenomenon may be explained by figure 15. For free GNPs and 
fluorophores, when the fluorophore concentration is fixed, the distance between the GNP and fluorophore is determined by the GNP concentration.

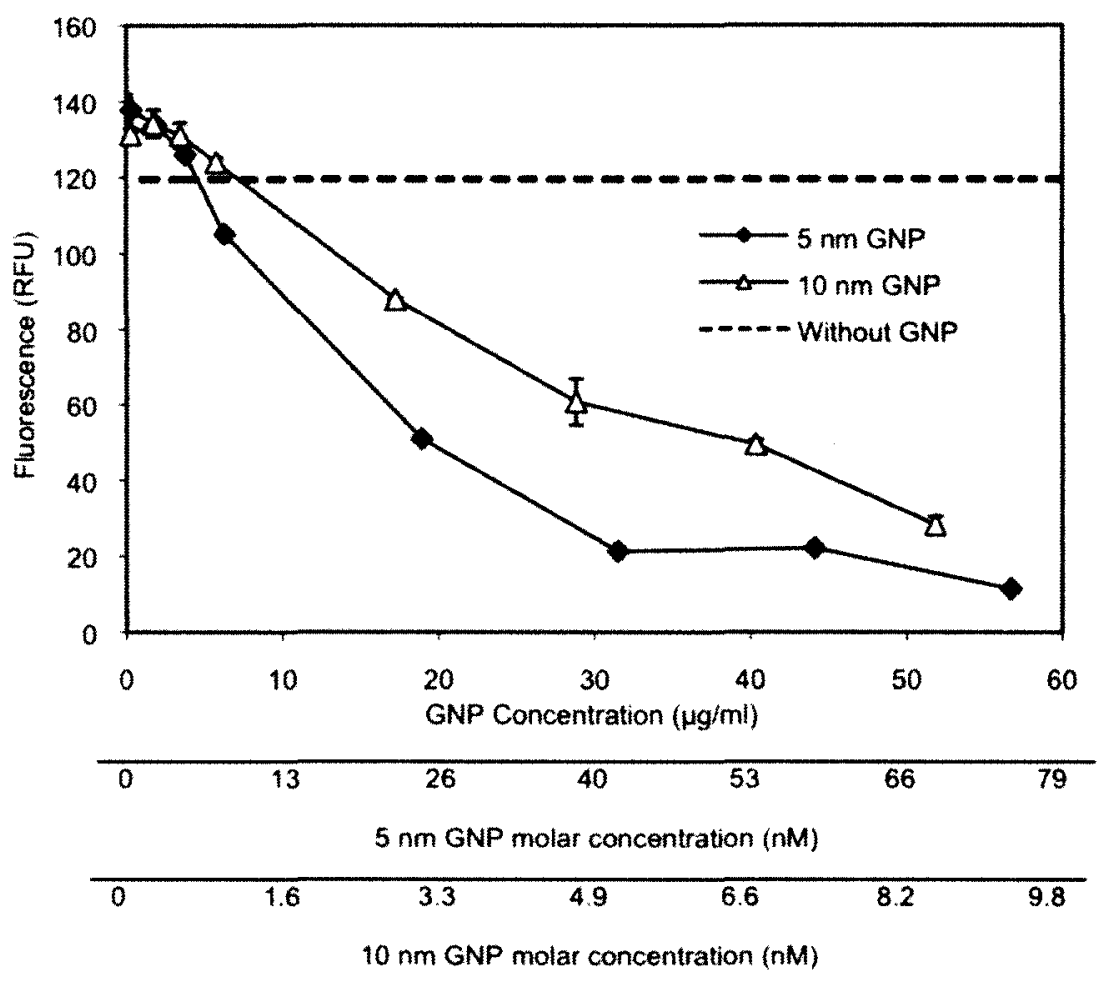

Figure 17. Fluorescence of free Cypate with various concentrations of free GNP (Cypate concentration for both GNP sizes $30 \mathrm{nM}$ ).

At concentrations greater than $6 \mu \mathrm{g} / \mathrm{ml}$ (for $5 \mathrm{~nm} \mathrm{GNP}, 8.3 \mathrm{nM}$, for $10 \mathrm{~nm} \mathrm{GNP}$, $1.0 \mathrm{nM})$, the fluorescence was quenched, and the quenching level increased with the increase in GNP concentration. At the same wt concentration, $5 \mathrm{~nm}$ GNPs showed greater quenching than those at $10 \mathrm{~nm}$, probably because the number of $5 \mathrm{~nm}$ GNPs is 8 times greater than that of $10 \mathrm{~nm}$ GNP, and the total surface area is about 2 times larger. Also, due to its smaller size, $5 \mathrm{~nm}$ GNPs would have much greater mobility than $10 \mathrm{~nm}$ GNPs would, resulting in greater probability for Cypate to be near the GNP. In addition, as shown in the theory section, smaller GNPs tend to cause quenching. 
When the GNP concentration is very low [Fig. 18(b), with one GNP], the average distance between Cypate and GNP is longer, and the fluorescnece is either slightly enhanced or unaffected. At a higher GNP concentration [Fig. 18(a), with three GNPs], the distance between GNP and Cypate is shorter and, thus, more Cypate molecules are near the GNP surface, resulting in more fluorescence quenching.
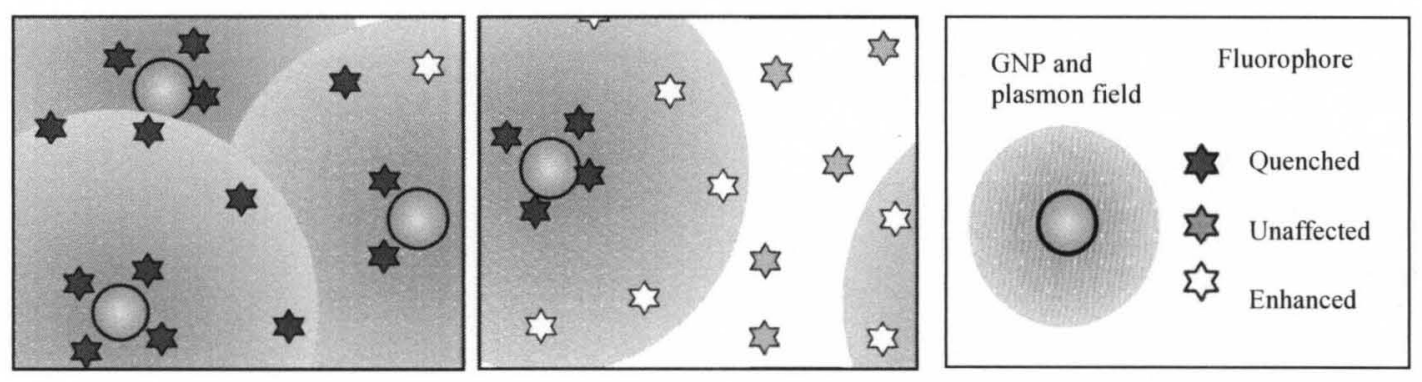

Figure 18. Schematic diagram for fluorescence of free Cypate affected by (a) high and (b) low GNP concentration in solution

As a preliminary study, this result verified that the GNPs affect Cypate fluorescence from quenching to enhancement, depending on the GNP concentration (distance between GNP and Cypate).

\section{Optimization of Spacers}

\section{(a) Short spacer}

Figure. 19 is a schematic diagram demonstrating the mechanism of the short spacer in the contrast agent of our interest. sPEGs are packed on the GNP surface for stabilizing the GNPs. Cypate is conjugated to GNP via the short spacer. The methods for conjugating Cypate to GNP via short spacer were: (1) Cypate (Cy) conjugated SSP (CySSP) was mixed with SPEG at a predetermined ratio and the mixture was reacted with GNPs to form a monolayer of two different molecules on the GNP surface, or (2) The 
$S S P$ with the $-\mathrm{ONH}_{2}$ ending ( $n S S P$ ) was mixed with sPEG at a predetermined ratio and the mixture was reacted with GNPs to produce GNP-nSSP. Then the m-Cypate reacted with BLK was reacted to the GNP-nSSP.

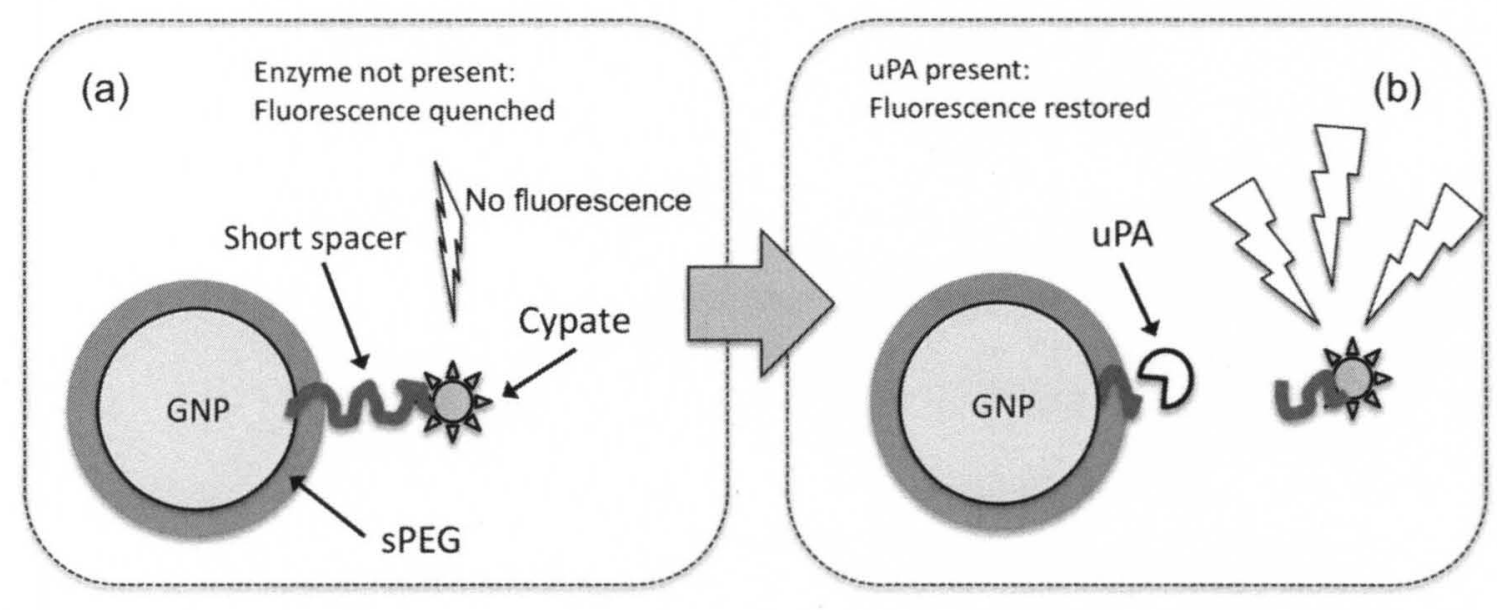

Figure 19. A schematic diagram of the uPA triggered fluorescence contrast agent, Cypate conjugated GNP via a short spacer: (a) normally emits little fluorescence (b) in the presence of uPA enzyme, the spacer is cleaved and the fluorescencing ability of the agent is restored.

For our system, since the short spacer and the fluorophore were already determined, the only parameter that could be varied was the GNP size. Theoretical study results suggested that smaller GNPs provided more effective quenching with the same distance between the GNP and the fluorophores. We, therefore, decided to first test relatively smaller GNPs, i.e. 3.7, 8.9 and $16.4 \mathrm{~nm}$ GNPs.

\section{(a-i) Characterization of GNP-SSP-Cy}

The GNP-SSP-Cy produced was characterized by UV-Visible spectroscopy to confirm the presence of both GNP and Cypate and to study their optical properties. Figure 20(a) shows the absorption spectra of GNP, SSP-Cy and GNP-SSP-Cy when 8.0 nm GNPs were used. GNPs and SSP-Cy show their signature absorption peaks at 520 and 
$780 \mathrm{~nm}$, respectively. GNP-SSP-Cy shows the peaks of both GNP and SSP-Cy. The peaks were slightly red-shifted, probably due to the changes in the refractive index of the GNPs after the polymer conjugation.

GNP-SSP-Cys were spread on TEM grid as a monolayer and dried under vacuum. TEM was performed to visualize the GNP core and potentially the coating [Fig. 20(b)]. The image shows a uniform GNP core size of $8.0 \mathrm{~nm}$. The sPEG coating is not visible because usually polymers do not respond to the electron beams well. Since TEM requires the sample to be dried and is performed in a high-vacuum, the image may not show the dispersion property of GNP-SSP-Cy in solution. Nonetheless, the particles are separated from each other at a constant distance, which is speculated to be the thickness of two, dry sPEG layers. Dynamic light scattering particle size analyzer was not used for this GNP product due to the interference caused by the Cypate fluorescence.

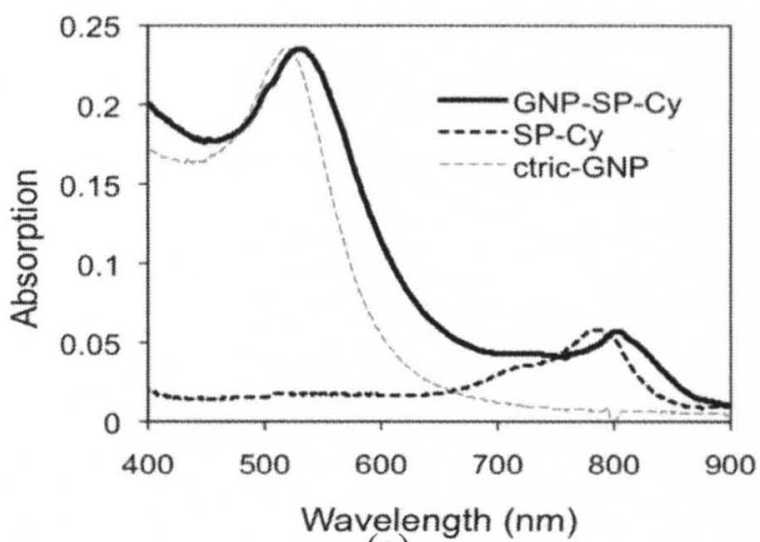

(a)

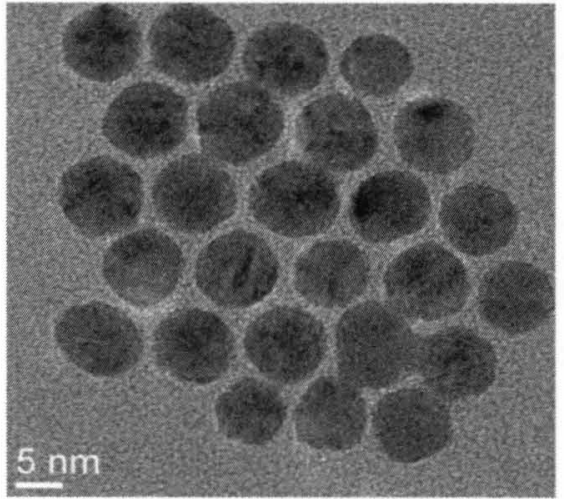

(b)

Figure 20. (a) Absorption spectra of GNP, SSP-Cy and GNP-SSP-Cy, and (b) TEM image of GNP-SSP-Cy [GNP, $8.0 \mathrm{~nm}$ ] 


\section{(a-ii) Effect of the GNP size on fluorescence quenching}

The fluorescence levels for the GNP-SSP-Cy products with GNP core size of 3.7, 8.0, $16.4 \mathrm{~nm}$, were observed and compared with that of SSP-Cy alone (Fig. 21). The Cypate concentration in all GNP-SSP-Cy samples was adjusted to $10 \mu \mathrm{M}$, using the absoption at $780 \mathrm{~nm}$. At this Cypate concentration, the GNP concentration determined by $520 \mathrm{~nm}$ absorption were $31,7,4.2 \mathrm{nM}$ for 3.7, 8.0 and $16.4 \mathrm{~nm}$ GNP, respectively.

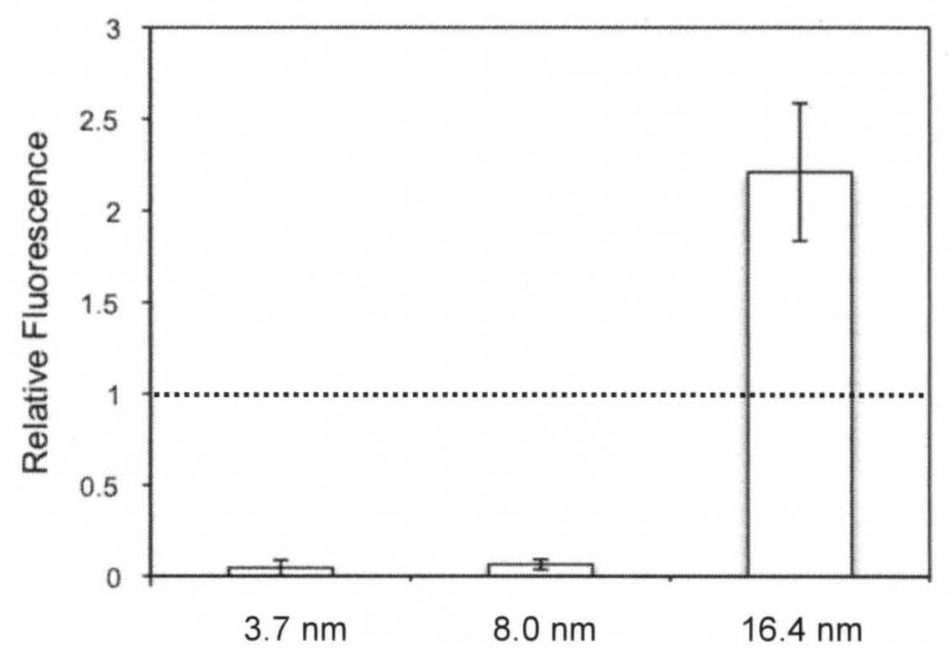

Figure 21. Relative fluorescence of the GNP-SSP-Cy with the core GNP size of $3.7,8.0$ and $16.4 \mathrm{~nm}$ at $10 \mu \mathrm{M}$ Cypate concentration in PBS buffer

For 3.7 and $8.0 \mathrm{~nm}$ GNPs, the fluorescence was quenched by $95 \%$ and $94 \%$, respectively. For $16.4 \mathrm{~nm}$ GNP, however, the fluorescence was enhanced by $120 \%$. Therefore, 3.7 and $8.0 \mathrm{~nm}$ GNPs were used in our further studies.

Figure 22 shows the theoretical estimation of the fluorescence of Cypate placed near a GNP of $3.7,8.0$ and $16.4 \mathrm{~nm}$. It shows that to obtain the fluorescence alteration level shown in Fig. 21, the spacer lengths should be $0.4,0.3$ and $1.5 \mathrm{~nm}$, for $3.7,8.9$ and $16.4 \mathrm{~nm}$ GNPs, respectively. They are much shorter than the SSP length that we 
estimated by the simulation, although the trend that bigger GNP results in higher fluorescence qualitatively agrees with the experimental results.

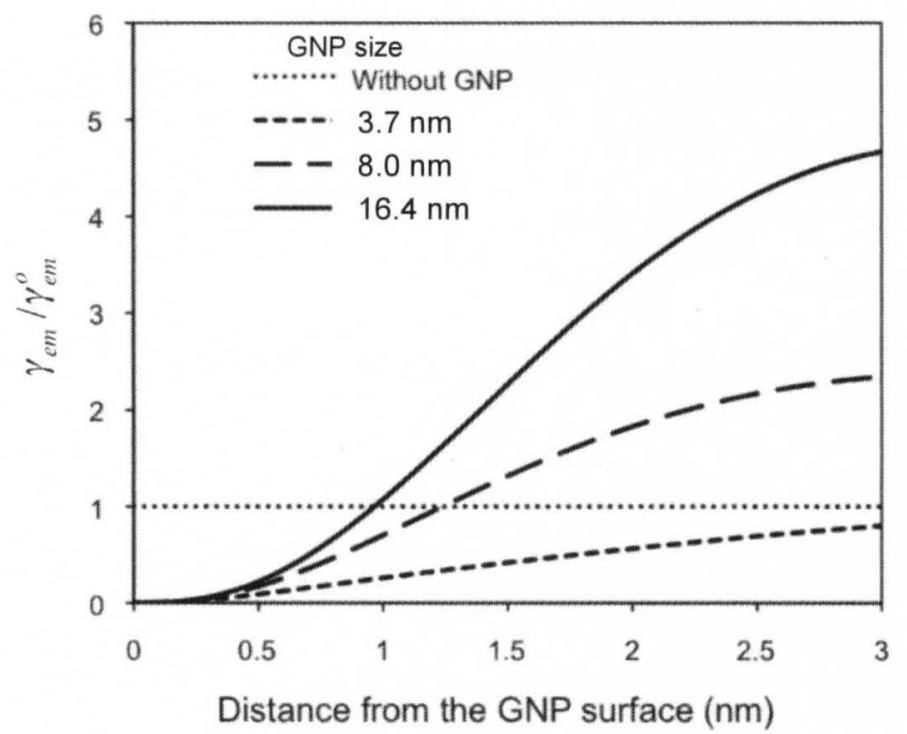

Figure 22. Theoretical estimation of fluorescence emission with the distance from the GNP surface for the GNPs sizes at $3.7,8.0$, and $16.4 \mathrm{~nm}$ for ICG $(\mathrm{Ex} / \mathrm{Em} 780 / 830 \mathrm{~nm})$

The discrepancy between the experimental result and theoretical prediction may be because:

(1) The length estimation for SSP was done by a molecular simulation assumes SSP to be extended configuration in vacuum, while in the actual system they were in a water based buffer and may not be linear.

(2) The theoretical result shown in Fig. 22 was for bare GNPs. In our experimental system, the GNPs were coated with sPEG, and Cypate is outside the sPEG layer, where the plasmon field may be slightly stronger than the one without sPEG coating (See Fig. 7). 
(3) The theoretical model is based on a system with a single fluorophore and a single GNP with no inter-particle or inter-fluorophore interference. In the experimental study, multiple fluorophores were conjugated to a single GNP, and multiple GNP-SSP-Cys were dispersed in the solution. There may be interaction among the particles and fluorophore molecules, e.g., self-quenching may occur among the Cys on the GNP and the fluorescence of GNP-SSP-Cy may be affected by the GNP of another GNP-SSP-Cy nearby.

(4) The theoretical analysis here was done only in one direction $(\theta=0)$, i.e., the effect of other directions were not considered, while, in the experimental system, Cypate is conjugated all around the GNPs. The field strength varies with $\theta$, thus the fluorescence alteration value is also different for different $\theta$.

The theoretical and experimental results, however, qualitatively agree in the sense that, at the same distance from the GNP surface, smaller particles provide less fluorescence.

\section{(a-iii) Conditional fluorescence restoration}

Our model system is to fluoresce only in the presence of uPA. The GNP-SSP-Cy products showing the fluorescence quenching (i.e., with 3.7 and $8.0 \mathrm{~nm}$ GNP cores) were tested with a sufficient amount of uPA (1030 units/ml), and their fluorescence changes were observed. Figure 23 shows the fluorescence levels of the GNP-SSP-Cy before and 5 minutes after uPA was applied. The fluorescence of 3.7 and $8.0 \mathrm{~nm}$ GNP-SSP-Cy was restored up to 21 and $43 \%$, respectively. 


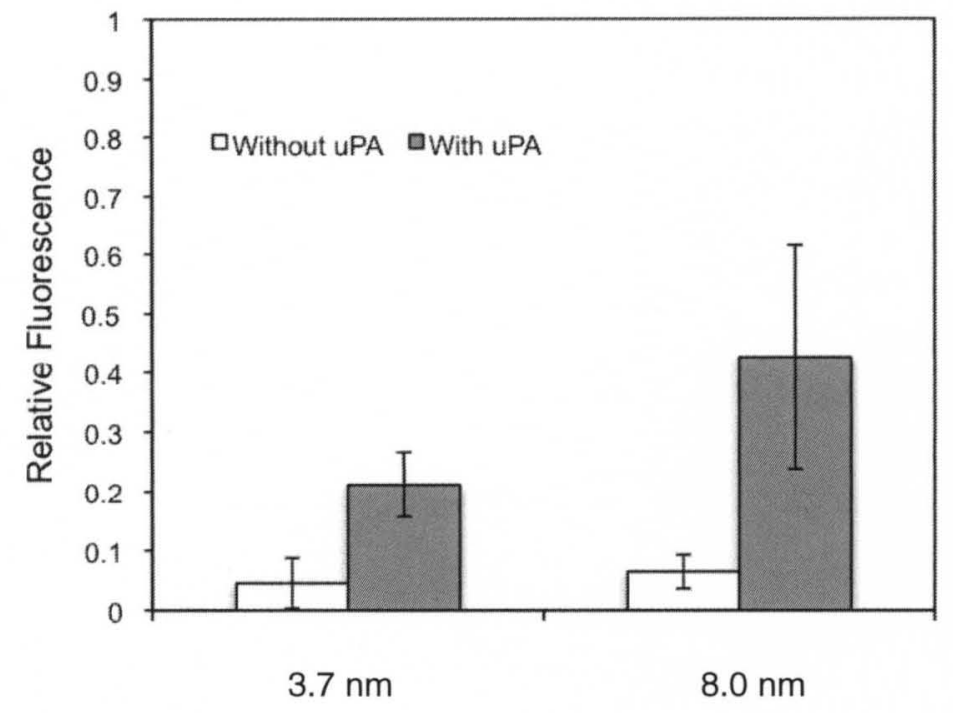

Figure 23. The relative fluorescence levels of GNP-SSP-Cy with 3.7 (GNP concentration $31 \mathrm{nM}$ ) and $8.0 \mathrm{~nm}$ (GNP concentration $7 \mathrm{nM}$ ) GNPs at $10 \mu \mathrm{M}$ Cypate in PBS buffer, before and after adding uPA. Reaction time: $5 \mathrm{~min}$; Ex/Em: 780/830 nm

Ideally, when uPA reacts with the GNP-SSP-Cy, the fluorescence is to be completely (100\%) restored. The possible reasons for this partial fluorecence restoration were: (i) the Cypate molecules freed from the GNPs would be still placed very close to GNPs because of the high GNP concentration and the fluorescence might be still quenched; (ii) SSP and sPEG molecules might be packed tightly on the GNP surface and uPA would not have easy access to the uPA-substrate site in the SSP. This may be resolved by replacing sPEG with shorter PEG molecules; and (iii) the released Cypate molecules might have been adsorbed at the hydrophobic site of sPEG.

We have first tested the hypothesis (i). The relative fluorescence of $10 \mathrm{~nm}$ GNP$n S S P-m C y$ before and after adding uPA was observed at various GNP concentrations, while the mCy/GNP ratio is fixed (performed by Dr. Robert Lupitskyy; Fig. 24). The 
GNP concentrations tested $(2.5,5$, and $10 \mathrm{nM})$ were in the range of the concentration used in our previous studies [Fig. $17(7 \mathrm{nM})]$.

The GNP-nSSP-mCy as it is, showed very little fluorescence $(<5 \%$ of the control). When uPA was applied to the sample, the fluorescence restoration for the GNP concentration of $2.5,5$, and $10 \mathrm{nM}$ were 71,42 and $12 \%$, respectively, indicating that the restoration rate is highly affected by the GNP concentration in the solution. We expect that at a lower GNP concentration the fluorescence will be fully restored. We were, however, not able to further lower GNP concentration, because at the GNP concentration at $2.5 \mathrm{nM}$, the $\mathrm{mCy}$ concentration reaches the lower limit $(\sim 40 \mathrm{nM})$ for a reliable fluorescence measurement.

This result suggested that the higher GNP concentration [i.e. (i) described above] may be the major cause for the low fluorescence restoration.

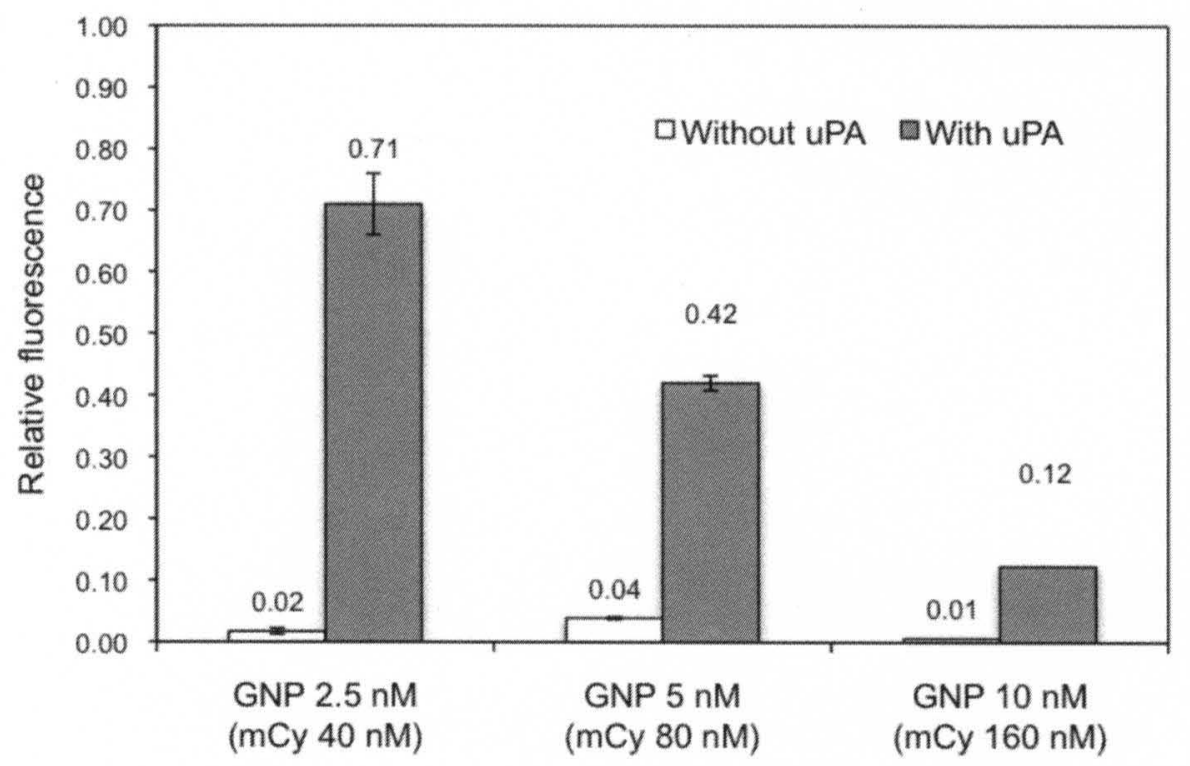

Figure 24. Fluorescence of GNP-nSSP-mCy with $8.0 \mathrm{~nm}$ GNP at various GNP concentrations, before and after adding uPA, Reaction time: $5 \mathrm{~min}$; Ex/Em: $780 / 830 \mathrm{~nm}$ 


\section{(b) Long Spacer}

The main role of the long spacer $(L \mathrm{SP})$ is to maximize the Cypate fluorescence for a GNP size selected. The theoretical results (Fig. 12) shows that the enhancement level is determined by the distance and the GNP size. For the spacers, the LSPs previously described (B-1. components of the contrast agent, b-ii) were tested for the Cypate fluorescence enhancement. For the GNP size, bigger GNPs provide a greater maximum enhancement and require a shorter distance for fluorescence quenching. For our system, however, the length of the short spacer was already fixed, and therefore, to maintain a satisfactory level of quenching the upper limit of the GNP size to be used was already determined. The GNP size to be tested for our system was therefore decided to be less than $20 \mathrm{~nm}(3.7,8.0$ and $16.4 \mathrm{~nm})$.

As previously described, sPEG is used for GNP surface modification and also for adjusting the spacing between the two adjunct fluorophores on the GNP surface. After testing several LSP-to-sPEG ratios, 1:9 was found to be working well without causing aggregation. The purified GNP-LSP was then reacted with m-Cypate (with BLK) to produce GNP-LSP-mCy.

\section{(b-i) Characterization of GNP-LSP}

According to our theoretical study results for the GNPs at a size range of 5-50 nm that enhance the ICG fluorescence, the distance between a GNP and ICG is in a range of 2-5 nm. Table 4 listed the spacers used in this study and their lengths estimated by a molecular simulation. Again, the mathematical model used to compute the length of the spacers assumes that the molecule is straight and in vacuum. 
Table 4. Spacers used and their estimated lengths by molecular simulation

\begin{tabular}{ccc}
\hline Spacers & Abbreviations & Extended Length $($ nm $)$ \\
\hline$H S-\left(\mathrm{CH}_{2}\right)_{1-}-\mathrm{PEG} \mathrm{G}_{3}-\mathrm{OH}$ & sPEG & $2.30^{*}$ \\
$\mathrm{HS}-\left(\mathrm{CH}_{2}\right)_{12}-\mathrm{PEG}_{4}-\mathrm{ONH}_{2}$ & $\mathrm{C} 12 \mathrm{PEG} 4$ & 3.20 \\
$\mathrm{HS}-\left(\mathrm{CH}_{2}\right)_{16}-\mathrm{PEG}_{4}-\mathrm{ONH}_{2}$ & C16PEG4 & 3.69 \\
$\mathrm{HS}-\left(\mathrm{CH}_{2}\right)_{12}-\mathrm{PEG}_{6}-\mathrm{ONH}_{2}$ & $\mathrm{C} 12 \mathrm{PEG} 6$ & 3.90 \\
$\mathrm{HS}-\left(\mathrm{CH}_{2}\right)_{16}-\mathrm{PEG}_{6}-\mathrm{ONH}_{2}$ & $\mathrm{C} 16 \mathrm{PEG} 6$ & 4.41 \\
$\mathrm{HS}-\left(\mathrm{CH}_{2}\right)_{12}-\mathrm{PEG}_{8}-\mathrm{ONH}_{2}$ & $\mathrm{C} 12 \mathrm{PEG} 8$ & 4.60 \\
\hline
\end{tabular}

${ }^{*}$ sPEG is the coating material

In an attempt to verify the spacer conjugation and also to know the actual length of the spacer, the GNPs conjugated with the SPs and sPEG were characterized by the dynamic light scattering (DLS) size analysis and TEM.

Table 5 shows the particle size measured by DLS, before and after the conjugation.

Table 5. Size of nanoparticles, before and after conjugating sPEG and sPEG/LSP, measured by light scattering particle size analyzer

\begin{tabular}{cccc}
\hline Sample & $\mathbf{5 n m G N P}$ & $\mathbf{1 0} \mathbf{n m ~ G N P}$ & $\mathbf{1 5} \mathbf{n m ~ G N P}$ \\
\hline Uncoated GNP & $3.7 \pm 0.9$ & $\mathbf{8 . 0} \pm 1.5$ & $16.4 \pm 0.90$ \\
\hline GNP-sPEG & $8.0 \pm 1.6$ & $10.2 \pm 0.9$ & $17.5 \pm 1.3$ \\
GNP-sPEG/C12PEG4 & $8.3 \pm 1.1$ & $11.2 \pm 1.2$ & $17.9 \pm 1.2$ \\
GNP-sPEG/C12PEG6 & $11.2 \pm 2.3$ & $10.5 \pm 1.7$ & $18.1 \pm 3.1$ \\
GNP-sPEG/C12PEG8 & $10.1 \pm 1.6$ & $11.5 \pm 1.9$ & $17.0 \pm 3.8$ \\
\hline
\end{tabular}

The diameter of the particles increased by $1.5-7.5 \mathrm{~nm}$ after coating. In general, the increase in diameter measured by DLS appeared to be much less than those theoretically estimated (6.4-9.2 nm, double of the length of the SPs), indicating that the actual length of the sPEG of spacers may be shorter than the estimation. In addition, the difference in the size between the GNP-sPEG and the GNP-sPEG/SPs was minimal, possibly because the majority molecules on GNP surface are sPEG. For bigger GNPs, the increase was less than the ones for smaller particles. DLS measures the hydrodynamic diameter of particles based on the diffusion of particles in the fluid. With the same polymer coating thickness, 
the movement of a particle with a bigger heavy (gold) core may be less affected by the light coating, results in less change in the particle size.

The particles were also imaged by TEM. Because TEM does not usually respond to organic polymer well, therefore, we put an extra effort to spread diluted GNP products on the TEM grid so that the particle solution formed a thin particle monolayer. The spacing between two adjacent particles was expected to be the thickness of two layers of spacers on GNP. Figure 25 shows the $8.0 \mathrm{~nm}$ GNPs conjugated with (a) $\mathrm{sPEG}$ and (b) sPEG and C12PEG6 spacer. The distance between two adjacent particles were measured at least 50 different arbitrarily selected locations and the mean distance was $1.2 \pm 0.1 \mathrm{~nm}$ for all samples. Again, no notable difference in layer thickness was observed among the GNPs coated with various spacers, probably because the polymer layer thickness in the TEM was represented mainly for SPEG. It should also be noted that the samples prepared for the TEM were dried under vacuum, and therefore, the actual thickness of the coating layer in aqueous solution may not be the same as the one shown in TEM images. A technique that can be used for this type of analysis is urgently needed for bio-nano research.

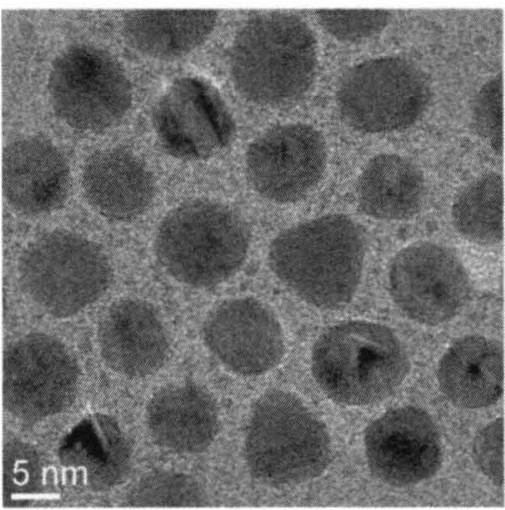

(a)

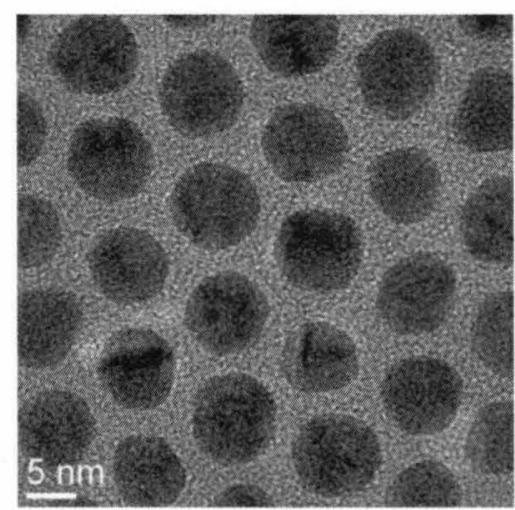

(b)

Figure 25. TEM image of $8.0 \mathrm{~nm}$ GNPs coated with (a) sPEG only and (b) sPEG and C12PEG6 


\section{(b-iii) Spectroscopic analysis of GNP-LSP-mCy}

GNP-LSP-mCy was characterized by UV-Vis spectrometry to confirm the Cypate conjugation. Figure 26 shows the absorption spectra of GNP, GNP-sPEG, GNPsPEG/LSP, GNP-LSP-mCy and mCy.

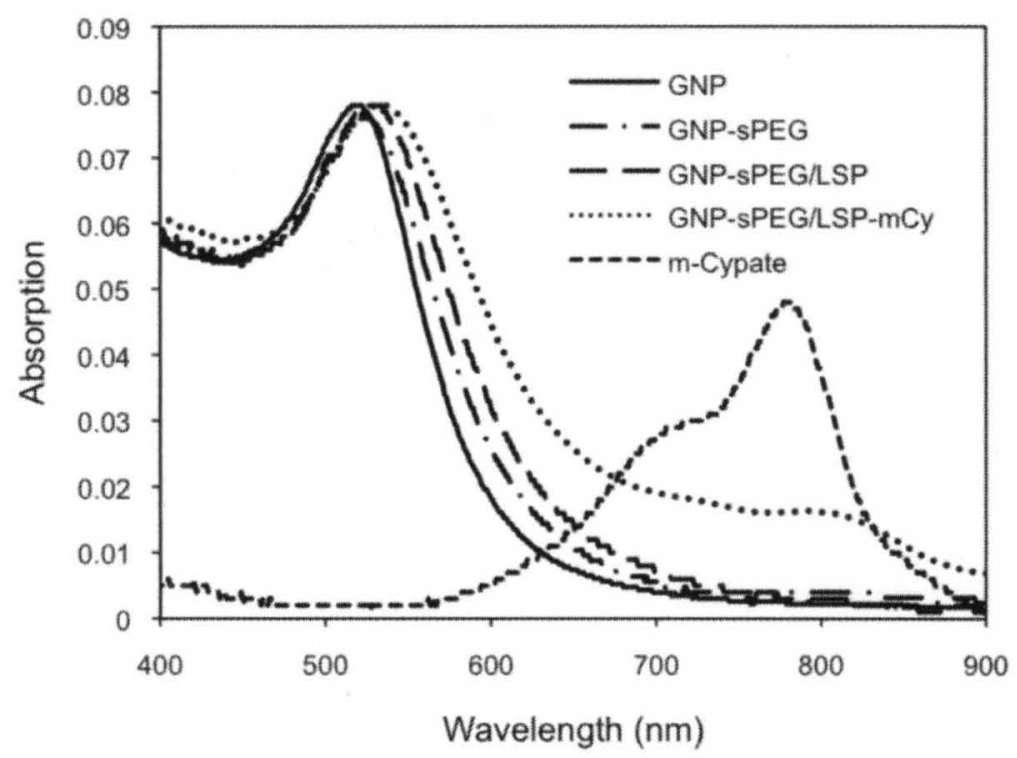

Figure 26. Absorption spectra of $8.0 \mathrm{~nm}$ GNP, GNP coated with sPEG alone, with sPEG and C12PEG6, GNP-LSP-mCy, and mCy

The absorption spectrum of GNP-sPEG is very similar to GNP except that the absorption peak was slightly red-shifted, caused by the changes in refractive index by the polymer coating. When the GNP is coated by both sPEG and LSP (C12PEG6), the peak showed a slightly greater red-shift. After reacting mCy onto the GNP via the LSP, the spectra showed both GNP and mCy absorption peaks. The concentrations of GNP and $\mathrm{mCy}$ were estimated by the absorption at their respective signature wavelengths. 


\section{(b-iv) Application of blocking agent for m-Cypate}

For binding Cypate to GNPs via the LSPs, initially mCy was reacted to GNP-LSP, as it was, without using BLK. After the product was purchased and tested for the fluorescence emission, strong fluorescence quenching was observed for all the LSPs. We speculated that the quenching might be caused by one or both of the following potential reasons (Fig. 27a): (i) $\mathrm{mCy}$ has two $-\mathrm{CHO}$ groups that can react to the $-\mathrm{ONH}_{2}$ group of the spacers. Therefore, it may react to two LSPs on the same GNP or on different GNPs, and the later may place GNP very close to $\mathrm{mCy}$, or (ii) Cypate is hydrophobic and it may be adsorbed to the hydrophobic $-\left(\mathrm{CH}_{2}\right)_{\mathrm{m}^{-}}$part of sPEG and LSPs (Fig. 27a), before or after it binds to LSPs. Thus the distance between the $\mathrm{mCy}$ and GNP surface becomes short and it leads to fluorescence quenching.

To resolve these issues, $\left(\mathrm{CH}_{3}\right)_{3} \mathrm{~N}^{+}-\left(\mathrm{CH}_{2}\right)_{2}-\mathrm{ONH}_{2}$ was used as the blocking agent. It was mixed with $\mathrm{mCy}$ at a 1:1 molar ratio, assuming that it blocks one of the two $\mathrm{CHO}$ groups of $\mathrm{mCy}$ so that each $\mathrm{mCy}$ reacts to only one LSP (Fig. 27b). Since BLK is hydrophilic, it is expected to increase the hydrophicility of $\mathrm{mCy}$ and thus helps avoid adsorption of $\mathrm{mCy}$ to the hydrophobic inner layer on GNP surface. With the use of BLK, the quenching problem disappeared and fluorescence enhancement with respect to spacer length and GNP size was observed instead of quenching. 

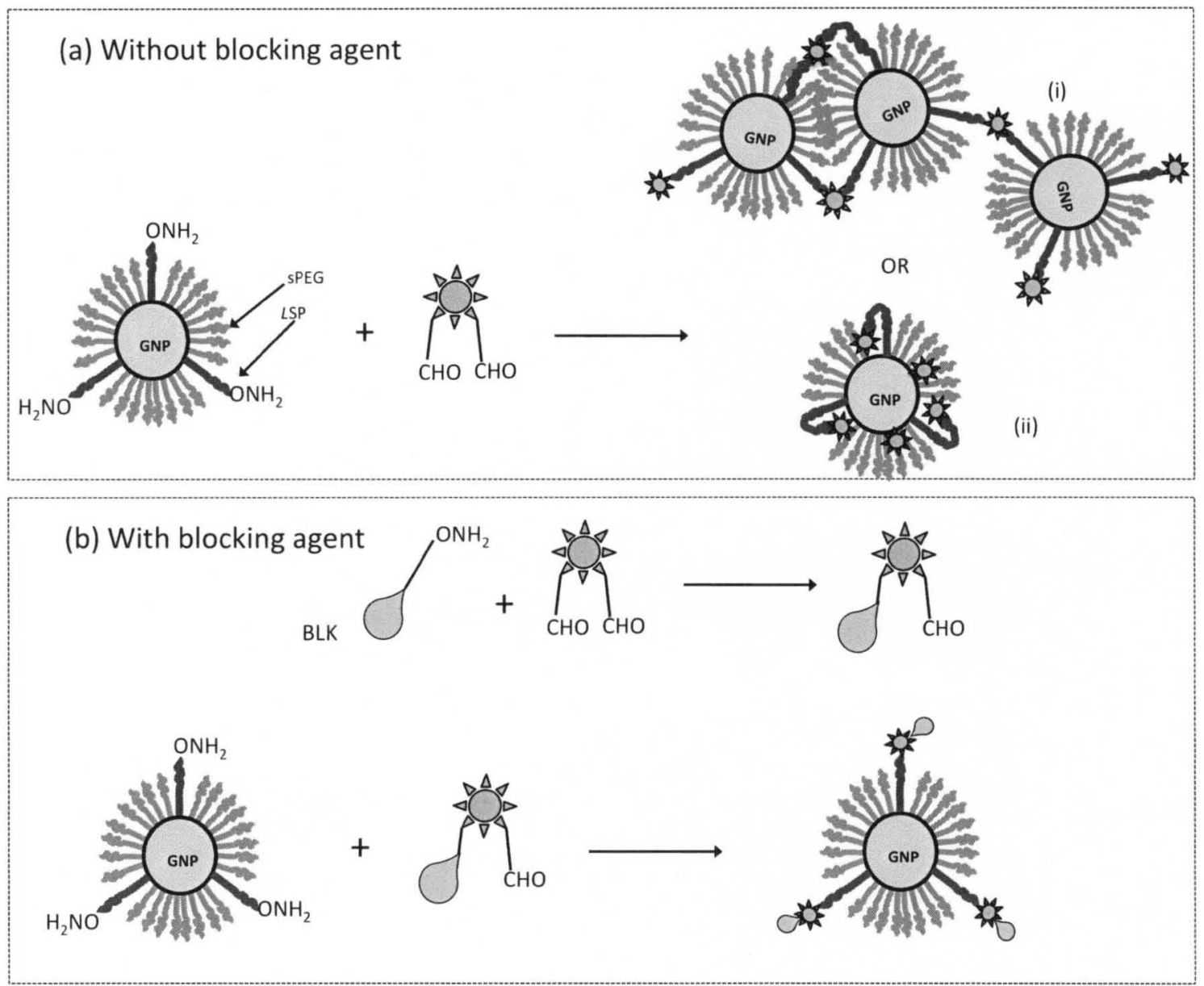

Figure 27. Schematic diagram of the function of blocking agent. (a) Without BLK (i) m-Cypate reacts to more than one LSPs and is placed very close to GNPs or (ii) m-Cypate is adsorbed to the hydrophobic section of sPEG and LSP. (b) One of the two - CHO group on m-Cypate is blocked therefore each LSP reacts to one m-Cypate, and m-Cypate is not adsorbed to the hydrocarbon chain close to the GNP surface.

\section{(b-v) Effect of $L S P$ length on fluorescence enhancement}

Figure 28 shows the relative fluorescence of the GNP-LSP-mCy at various LSP lengths [HS- $\left(\mathrm{CH}_{2}\right)_{\mathrm{m}}-\mathrm{PEG}_{\mathrm{n}}-\mathrm{ONH}_{2}, \mathrm{~m}=12$ or $16, \mathrm{n}=4,6$, or 8 ; 3.2-4.6 nm], when $8 \mathrm{~nm}$ GNP was used.

The shortest spacer, C12PEG4 (simulated length estimation, $3.2 \mathrm{~nm}$ ), resulted in a fluorescence quenching by $14 \%$. For C16PEG4 $(3.7 \mathrm{~nm})$, the fluorescence was enhanced 
by $36 \%$. The fluorescence of GNPs with C12PEG6 (3.7 nm) and C16PEG6 (3.9 nm) showed the enhancement of two times. For the spacers longer (C12PEG8, $4.6 \mathrm{~nm})$ than these two the fluorescence enhancement started to decrease.

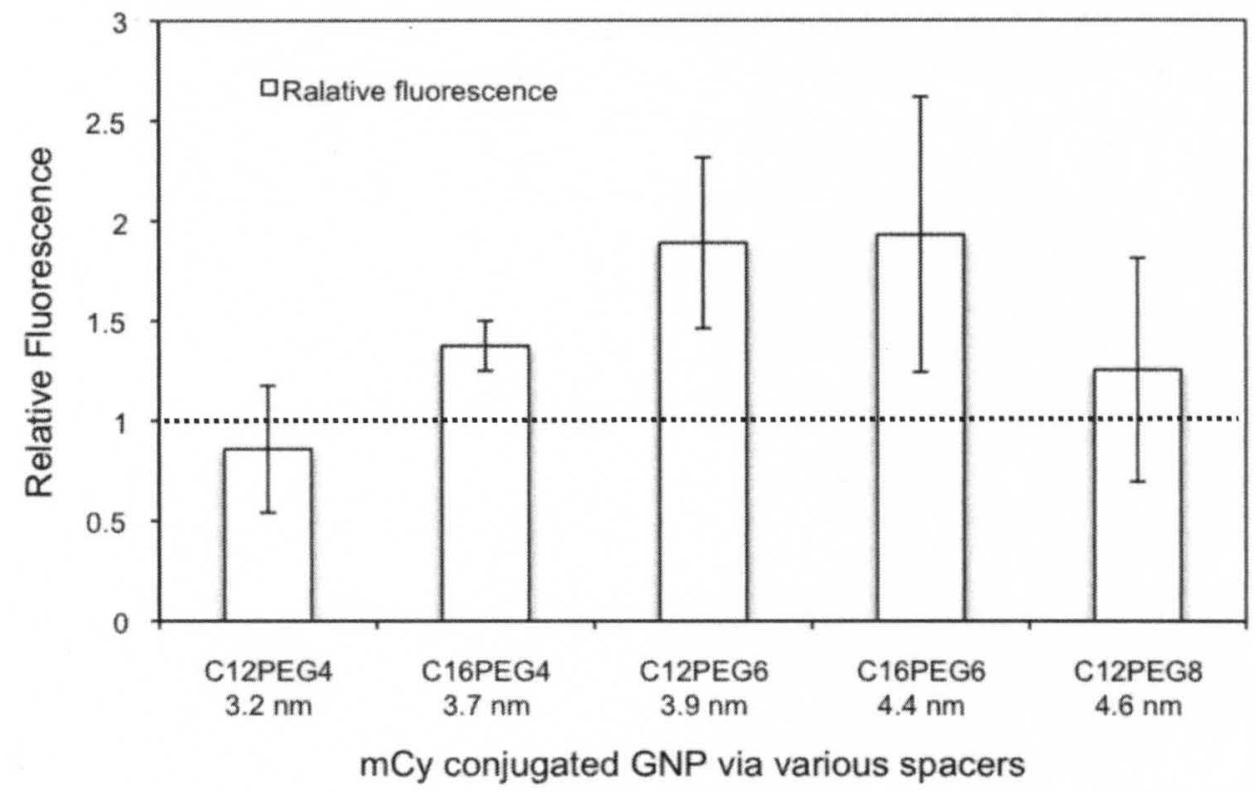

Figure 28. Relative fluorescence of mCy conjugated to $8.0 \mathrm{~nm}$ GNP via spacers with various lengths

When the results of theory and experiment were compared (Fig. 29), the level of the maximum enhancement was similar ( $\sim 2$ times). However, the distance showing the maximum in the theoretical result was slightly shorter than the corresponding experimental spacer lengths. If the theoretical values were shifted by $1 \mathrm{~nm}$ (dotted line), they fit to the experimental values well except the value for the spacer C12PEG8. The reason for this discrepancy may be that the spacer length in the aqueous environment may be slightly different from those estimated by the simulation with the assumption that the molecule is straight and in vacuum. 


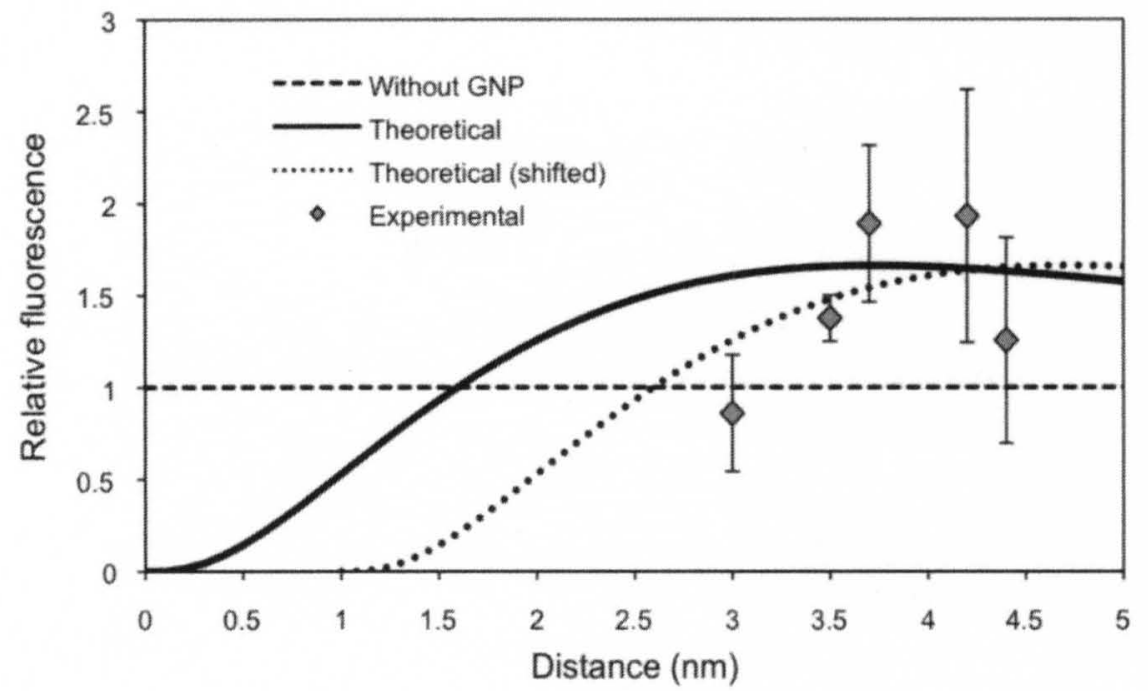

Figure 29. Relative fluorescence of mCy-BLK conjugated to $8.0 \mathrm{~nm}$ GNP via the spacers with various lengths, compared to the theoretically estimated fluorescence.

\section{(b-v) Effect of GNP size on fluorescence enhancement}

Theoretical study results show that, in general, bigger GNPs provide the greater fluorescence enhancement. We experimentally tested the changes in the Cypate fluorescence caused by three different GNP sizes $(3.7,8.0$ and $16.4 \mathrm{~nm})$. For this study, the spacers, C12PEG4 (3.2 nm), C12PEG6 (3.9 nm) and C12PEG8 (4.6 nm) were used.

Figure 30 shows both theoretical and experimental results of fluorescence alteration by $3.7,8$, and $16.4 \mathrm{~nm}$ GNP.

When $3.7 \mathrm{~nm}$ GNPs were used, the Cypate fluorescence was quenched for all spacers. The shortest spacer, C12PEG4 $(3.2 \mathrm{~nm})$, resulted in the most quenching $(60 \%)$ as expected, and C12PEG6 (3.9 nm) and C12PEG8 (4.6 nm) showed the quenching of 30 and $40 \%$, respectively. These results indicates that the $3.4 \mathrm{~nm}$ GNP may be too small to enhance the Cypate fluorescence, but it can be used as an effective quencher, and a shorter spacer may be desired for greater quenching. The experimental results 
qualitatively agreed with the theoretical predication, although the two results fit better when the theoretical result is shifted by $1 \mathrm{~nm}$.
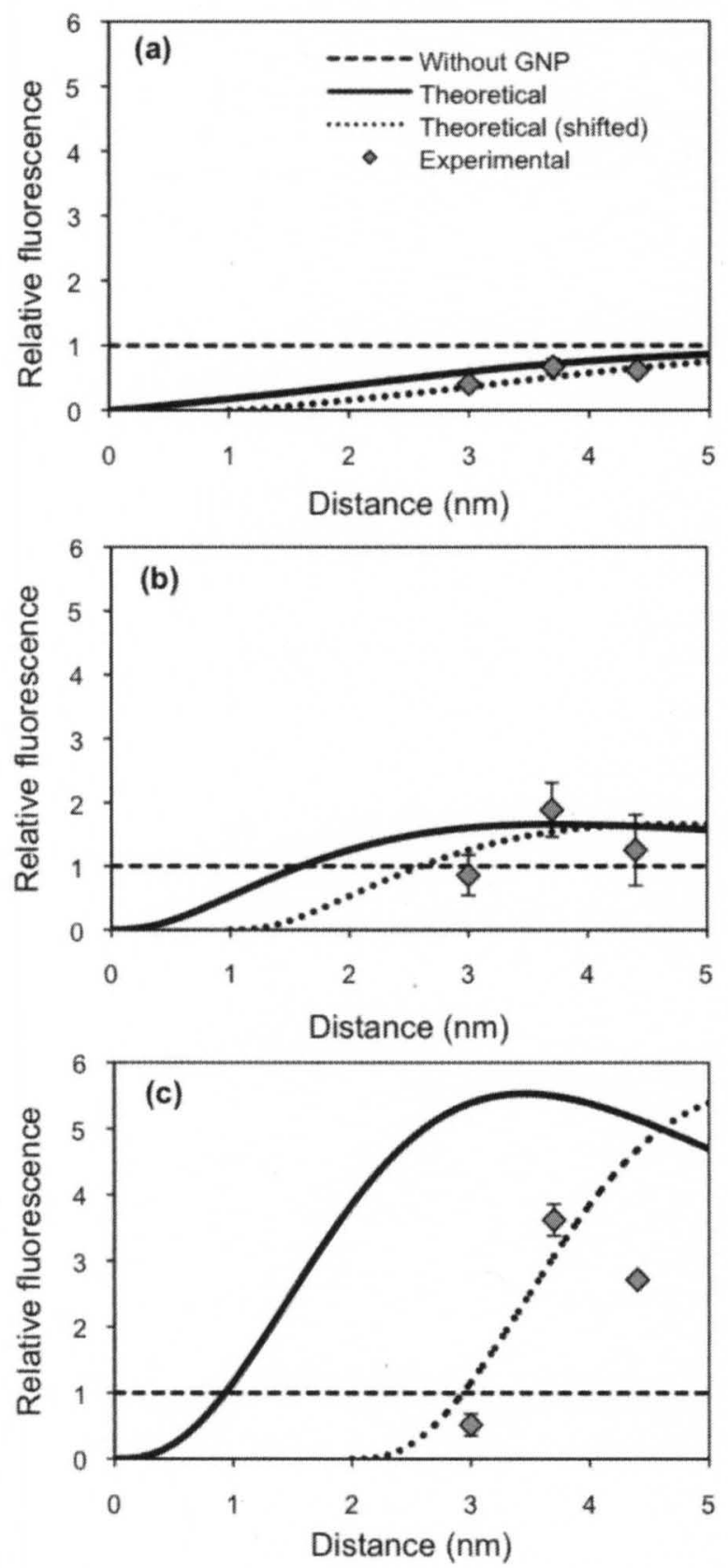

Figure 30. Relative fluorescence emjamcement of $\mathrm{mCy}$ conjugated to (a) 3.7 (b) 8.0 and (c) $16.4 \mathrm{~nm}$ GNPs via spacers with various lengths, compared to the theoretically estimated fluorescence level.

For $8.0 \mathrm{~nm}$ GNP, the result has already been discussed.

With the $16.4 \mathrm{~nm}$ GNP, C12PEG4 caused 50\% quenching, while C12PEG6 and C12PEG8 provided the enhancement of 3.6 and 2.7 times, respectively, greater than that 
by the $8.0 \mathrm{~nm}$ GNP (2 times). When it is compared to the theoretical results, the magnitudes of fluorescence changes by the spacers C12PEG4 and C12PEG6 are similar to those by the theoretical results that are shifted by $2 \mathrm{~nm}$ in the $\mathrm{x}$-axis. However, the one with C12PEG8 was significantly lower than the theoretical value.

Both theoretical and experimental results confirmed that for fluorescence enhancement, GNP size needs to be sufficiently large, and bigger GNPs provide the greater maximum enhancement. The results also show that the spacer length needs to be carefully selected to obtain the maximal enhancement. When the experimental results were compared to the theoretical values, the estimated length of the spacer used in the experiment were 1-2 nm longer than those of the theory. This phenomenon was observed in the short spacer study and previous studies from the Kang group [Kang, et al., 2011; Wang, et al., 2011], and the possible causes for the discrepancy have been discussed in the short spacer study. Although the exact reasons for this discrepancy were not fully understood yet, the difference is not significant. Therefore, the theoretical prediction is still very valuable in selecting the proper parameters for developing appropriate contrast agents using GNP, which reduces the number of experiments significantly.

In the process of designing and finalizing our dual spacer an important constraint is that the conditions need to satisfy both short and long spacer performance. Although $16.4 \mathrm{~nm}$ GNP provides the greatest fluorescence enhancement, it does not quench the fluorescence with the short spacer. Therefore, for the dual spacer system, $8.0 \mathrm{~nm} \mathrm{GNP}$, which provides quenching and enhancement with the SSP and LSP respectively, was decided to be used. 


\section{(c) Dual-spacer}

\section{(c-i) Design of the dual-spacer}

In the original design of the dual spacer (Fig 31), one of the two - $\mathrm{COOH}$ groups of Cypate is conjugated to a short spacer and the other, to a long spacer (DSP-Cy). Ideally, Cypate is to be connected to GNP by both spacers as shown in Fig. 31. However, the design may easily produce several undesired products as shown in Fig. 32, especially because there is a difference in length of the two spacers. Sometimes only one spacer may be attached to a GNP and the other spacer is free. Some times two spacers are attached to two different GNPs and multiple GNPs are connected by the spacers (crosslinking). Slight cross-linking may be tolerable, because fluorescence will be quenched as long as Cypate is conjugated to GNP via the short spacer, and at least it does not emit non-specific fluorescence. Severe cross-linking may result in the GNP aggregation.

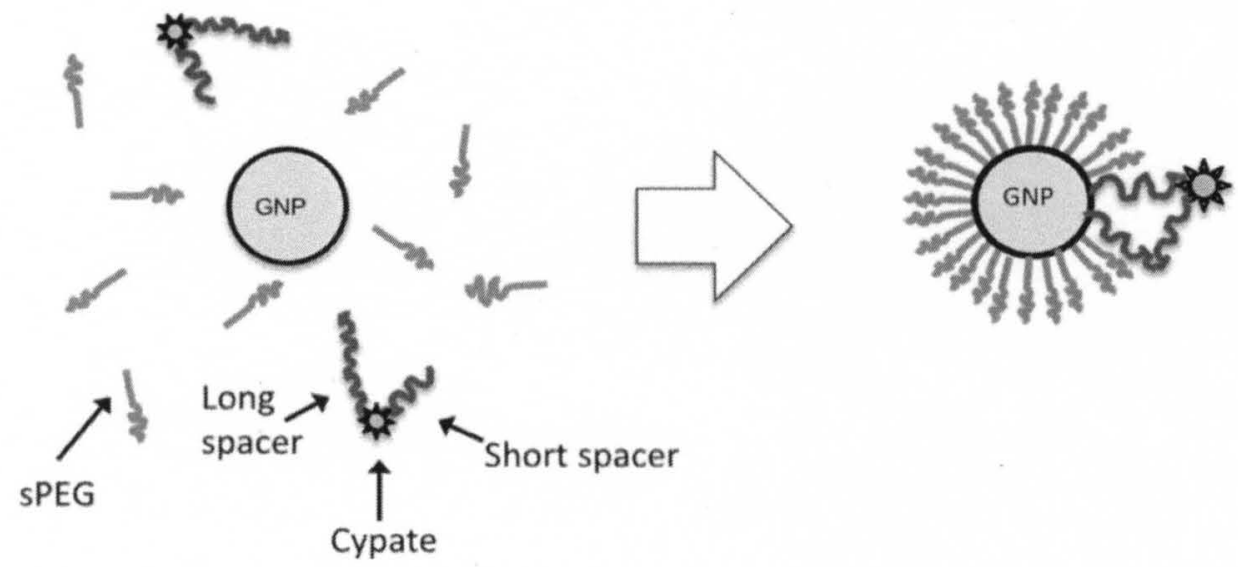

Figure 31. Original design for the synthesis of GNP-DSP-mCy (ideal case) 
(a)

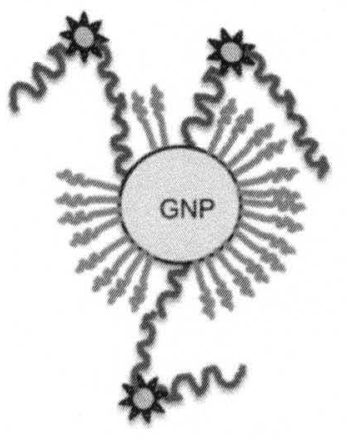

(b)

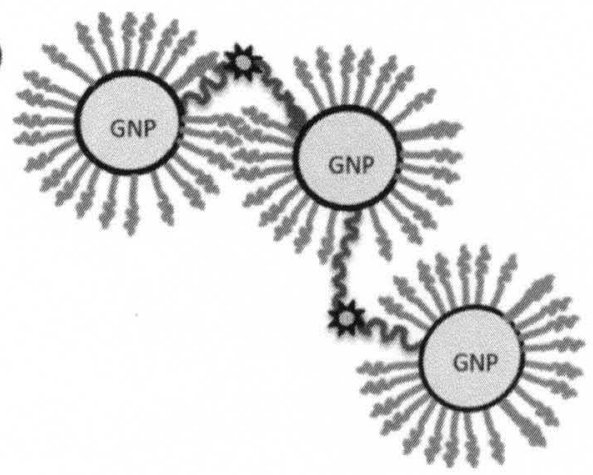

Figure 32. Potential undesired product (a) incomplete conjugation (b) cross linking from the original design for the synthesis of GNP-DSP-mCy

In addition, the synthesis of DSP-Cy has been difficult. The Nantz group has attempted to synthesize the DSP-Cy via the approaches of (1) solid-phase amide coupling, (2) solid-phase oximation coupling, and (3) solution-phase oximation coupling (from their report to DOD). None of them were successful due to the damage in Cypate molecule during the purification process.

We, therefore, took a new approach to resolve these issues: A ring-shaped dual spacer ( $r D \mathrm{SP}$ ) was designed (Fig. 33). The design was commissioned to a company to produce it (NeoGroup Inc., Cambridge, MA). In the $r D S P$, the two ends of long spacer are attached to the two ends of the short spacer, making a circular shape. The rDSP has one single $-\mathrm{SH}$ group for reacting to GNP surface, and a single $-\mathrm{ONH}_{2}$ group for reacting with m-Cypate. This rDSP can be used to connect m-Cypate to GNP in the same manner that the $n S S P$ or $L S P$ does. 


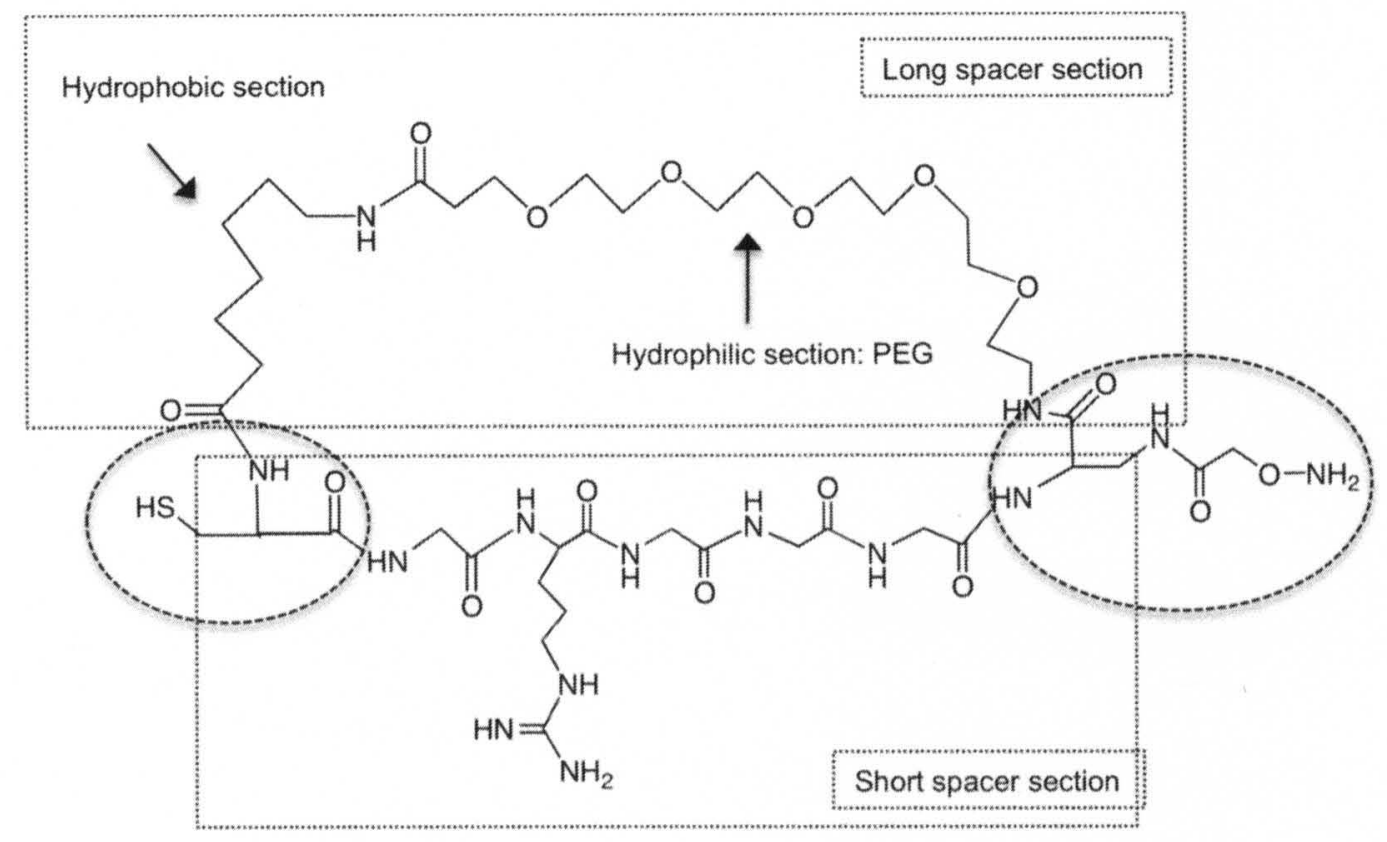

Figure 33. The chemical structure of the ring-shaped dual spacer (rDSP)

To facilitate the synthesis of the $r D \mathrm{SP}$, the structures of the short and long spacers were slightly modified. Our short spacer was $\mathrm{HS}-\left(\mathrm{CH}_{2}\right)_{2}$-NHC(O)-GGRGGG$\mathrm{C}(\mathrm{O})\left(\mathrm{CH}_{2}\right)-\mathrm{ONH}_{2}$. Here, the following modifications were performed: at the $-\mathrm{SH}$ end, HS- $\left(\mathrm{CH}_{2}\right)_{2}-\mathrm{NH}-$ was replaced by $\mathrm{HS}-\mathrm{CH}_{2}-\mathrm{CH}\left(\mathrm{NH}_{2}\right)-\mathrm{C}(\mathrm{O})$ - which provides a side group for connecting the new long spacer section later. At the - $\mathrm{ONH}_{2}$ end for Cypate reaction, $\mathrm{CH}_{2}-\mathrm{ONH}_{2}$ was replaced by $-\mathrm{NH}-\mathrm{CH}(\mathrm{COOH})-\mathrm{CH}_{2}-\mathrm{NH}-\mathrm{C}(\mathrm{O})-\mathrm{CH}_{2}-\mathrm{O}-\mathrm{NH}_{2}$, which has a side group $-\mathrm{COOH}$ for connecting the long spacer later. However, it introduces extra length [-CH(COOH)- $\left.\mathrm{CH}_{2}-\mathrm{NH}-\mathrm{C}(\mathrm{O})-\right]$. To keep the length of the short spacer to be short for quenching while maintaining the peptide substrate function, we removed one Glycine in the peptide sequence, having -GRGGG- instead of -GGRGGG-.

Our long spacer was HS- $\left(\mathrm{CH}_{2}\right)_{12}-\left(\mathrm{OCH}_{2} \mathrm{CH}_{2}\right)_{6}-\mathrm{ONH}_{2}$. In the new design, HS$\mathrm{CH}_{2}-\mathrm{CH}(\mathrm{COOH})-\mathrm{NH}_{2}$ provides the -SH group, which gives and extra length of $-\mathrm{CH}_{2}$ $\mathrm{CH}_{2}(\mathrm{COOH})-\mathrm{NH}-$. For the $-\mathrm{ONH}_{2}$ end, using $\mathrm{H}_{2} \mathrm{~N}-\mathrm{CH}(\mathrm{COOH})-\mathrm{CH}_{2}-\mathrm{NH}-\mathrm{C}(\mathrm{O})-\mathrm{CH}_{2}-\mathrm{O}$ - 
$\mathrm{NH}_{2}$ results in extra length of $\mathrm{C}(\mathrm{O})-\mathrm{CH}_{2}-\mathrm{CH}_{2}-\mathrm{NH}-\mathrm{C}(\mathrm{O})-\mathrm{CH}_{2}$. Hydrocarbon chain and PEG are connected through amide-bond, which also introduce extra length [-NH-C(O)-]. To compromise these length increases and maintain the total length similar to our long spacer, the $-\mathrm{CH}_{2}$ - number was reduced from 12 to 6 , and EG number was reduced from 6 to 5 .

\section{(c-ii) Characterization and performance of the $r D S P$}

The absorption spectra of GNP, m-Cypate and GNP-rDSP-mCy are shown in Figure 34. The GNP-rDSP-mCy showed both GNP and $\mathrm{mCy}$ absorption peaks, confirming the conjugation.

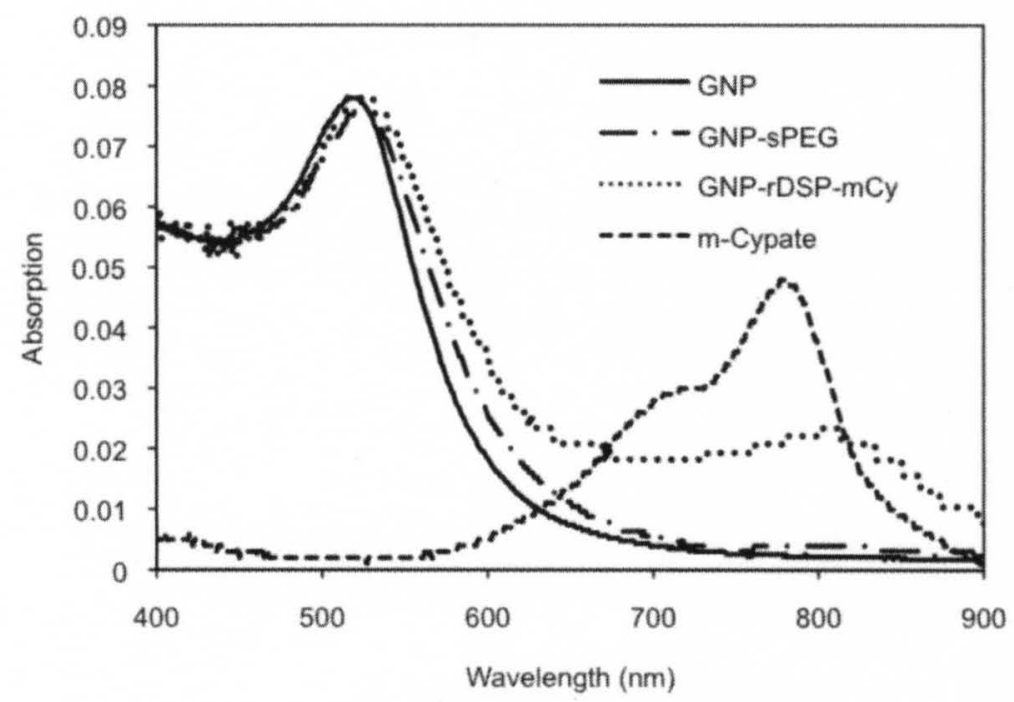

Figure 34. Absorption spectra of $8.0 \mathrm{~nm}$ GNP, GNP coated sPEG, GNP$r D \mathrm{SP}-\mathrm{mCy}$, and $\mathrm{mCy}$

Figure 35 shows the relative fluorescence of GNP-rDSP-mCy (GNP size, $8.0 \mathrm{~nm}$ ) and after uPA was applied. 


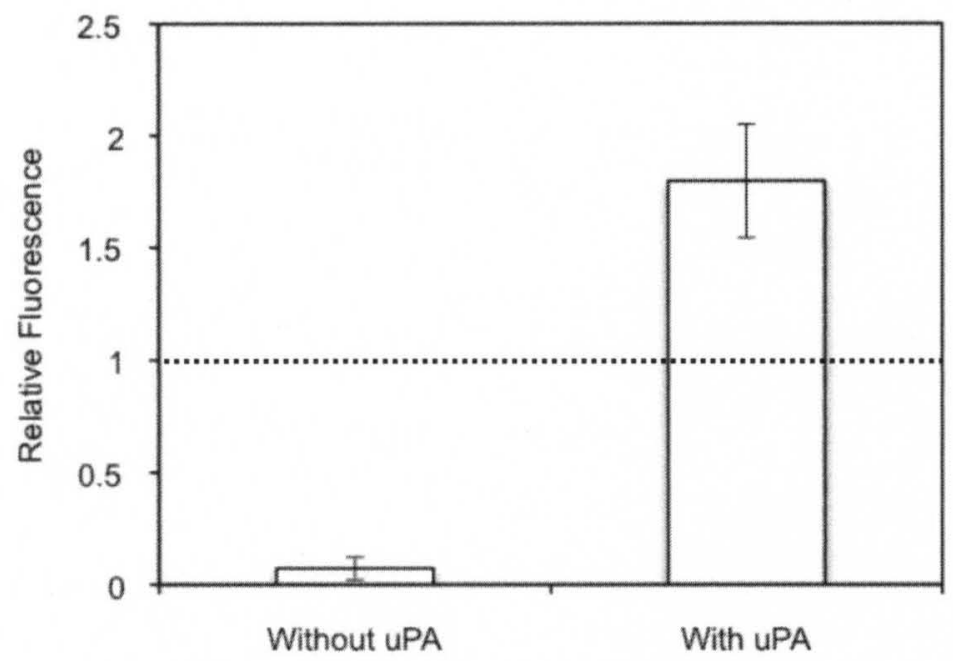

Figure 35. Fluorescence of GNP-rDSP-Cy ( $8.0 \mathrm{~nm}$ GNP) at $100 \mathrm{nM}$ Cypate, $0.9 \mathrm{nM}$ GNP, before and after adding uPA at 1030 units $/ \mathrm{ml}$ $(\mathrm{Ex} / \mathrm{Em} 780 / 830 \mathrm{~nm})$

The fluorescence of GNP-rDSP-mCy was quenched by $93 \%$, very close to the one with GNP-SSP-Cy (94\%). When uPA was added, the fluorescence increased to 1.8 times of the m-Cypate, which is slightly lower than the enhancement for the $8.0 \mathrm{~nm}$ GNP with the $L \mathrm{SP}$ (2 times).

In summary, with $8.0 \mathrm{~nm}$ GNP, the $r D S P$ provided the fluorescence quenching of $93 \%$, and the fluorescence enhancement of 1.8 times, upon uPA addition. This result confirms the functionality of the design of the novel contrast agent utilizing both fluorescence quenching and enhancement by GNPs.

\section{Cell Study}

\section{(a) Short spacer performance}

The GNP-SSP-Cys showing satisfactory performance of the fluorescence quenching, and the restoration with uPA addtion (in PBS buffer) were tested for the 
selected breast cancer cell lines. Two cell lines tested were: MCF-7 cells known to secrete little uPA (uPA-) as a control, and MDA-MB-231 cells expressing high level of uPA $(\mathrm{uPA}+) . \mathrm{uPA}+$ cells produce and release uPA from the cells to the surrounding media. Therefore, the uPA concentration in the media increases with time. Here the cells were cultured in serum-free media and incubated for a predetermined time to let the uPA accumulate in the media.

\section{(a-i) uPA secretion of the cell lines}

To see the feasibility of our contrast agent in cell culture systems, before testing the GNP-SSP-Cy, the uPA concentration and its activity of the cell lines in the media needed to be confirmed. The cell study was done in the Bates group at University of Louisville, with the help of the group members (Dr. Tariq Malik and Ms. Levona Coxon). The uPA concentrations in the media were measured 8 and $32 \mathrm{hrs}$ (arbitrarily chosen) after the cells were plated. The uPA quantification was done by enzyme-linked immunosorbent assay (ELISA). At 8 hrs, the uPA concentrations in both cell lines were at the lower limit of the ELISA, with a high noise level generated among the 4 duplicates of the sample (Fig. 36). At 32 hrs, MCF-7 cell line (uPA-) showed a low level $(0.76 \pm 0.65$ $\mathrm{ng} / \mathrm{ml})$ of uPA. Nevertheless there was uPA in the medium. The MDA-MB-231 (uPA+) cell line showed the uPA concentration of $3.15 \pm 1.20 \mathrm{ng} / \mathrm{ml}$. This concentration was approximately 1000 times lower than the concentration used in our experiments in PBS buffer $(2 \sim 10 \mu \mathrm{g} / \mathrm{ml})$. 


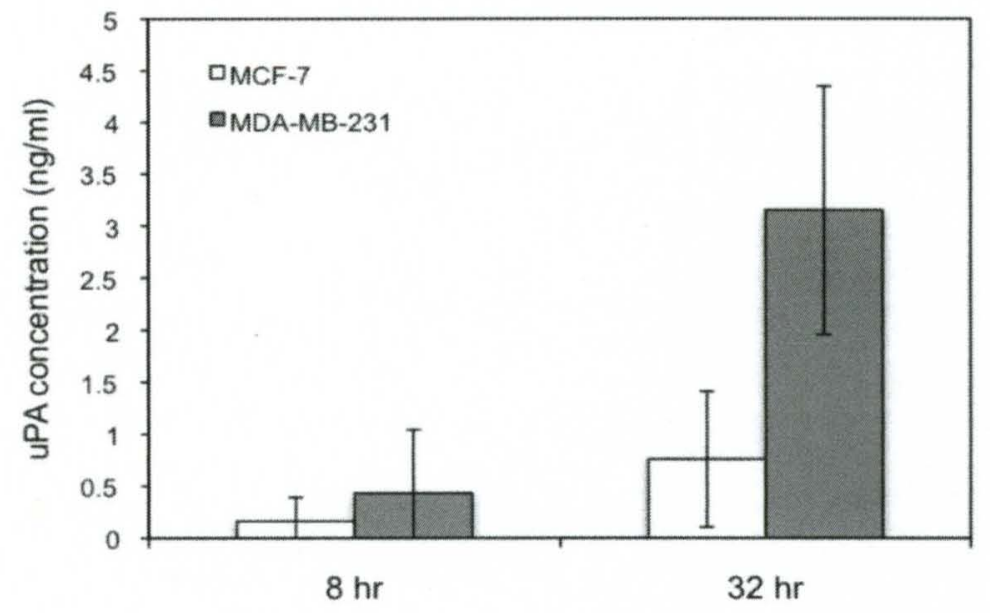

Figure 36. uPA concentrations in the media containing MCF-7 (uPA-) or MDA-MB-231 (uPA+) cells, after 8 and 32 hrs of cell culturing

\section{(a-ii) Fluorescence change of GNP-SSP-Cy in cell lines}

The GNP-SSP-Cy with $8.0 \mathrm{~nm}$ GNP core was tested in MCF-7 (uPA-) and MDAMB-231 (uPA+) cell lines. This experiment was performed by Dr. Sharon Bloch in the Achilefu group at Washington University. The cells were plated and cultured for 32 hours to have a sufficient amount of uPA. GNP-sSP-Cy samples at $10 \mu \mathrm{M}$ Cypate concentration were added to the media containing cells and incubated for $2 \mathrm{hrs}$ assuming that longer reaction time will provide enough reaction time for lower uPA concentration. Since the cleaving of short spacer should takes place in the cell culture medium where the uPA is present, the fluorescence from the media was monitored (Fig. 36). For this study, a Pear Imager (Licor) was used to measure the fluorescence, with excitation $780 \mathrm{~nm}$ and the emission was detected from $805-830 \mathrm{~nm}$.

The fluorescence of GNP-SSP-Cy in the media without cells was measured as a control, and the fluorescence was at the noise level (5.2 RFU). In the media from the MCF-7 (uPA-) cell culture, the fluorescence became very high (183 RFU), indicating the 
presence of uPA or some other biomolecules that cleaves the short spacer in the cell media (as shown in the ELISA result).

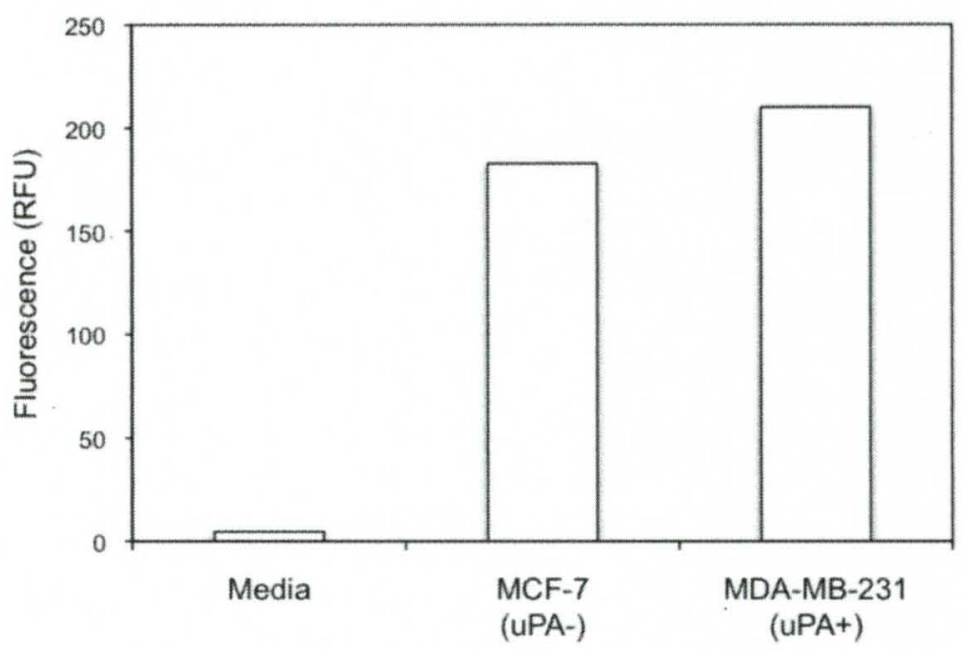

Figure 37. Fluorescence of GNP-SSP-Cy in media without cells, with MDA-MB-231 cells, and with MCF-7 cells after $2 \mathrm{hr}$ incubation. Ex/Em: $780 / 805-830 \mathrm{~nm}$

It is speculated that, a very long reaction time ( $2 \mathrm{hrs})$ allowed the low level of uPA in MCF-7 to react with the short spacer. In the media of MDA-MB-231 cells the fluorescence became $210 \mathrm{RFU}, 15 \%$ higher than the one in the media of MCF-7 (uPA-) cells. For further studies, a study for the time dependent fluorescence restoration is suggested to be performed.

\section{(b) Fluorescence of GNP-LSP-mCy in cell lines}

The well performing GNP-LSP-mCy with the $8.0 \mathrm{~nm}$ GNP core showed the fluorescence level twice of that of the mCy alone (Fig. 27). The well performed GNPLSP-mCy was tested in cell lines of MCF-7 and MDA-MB-231, by Dr. Sharon Bloch in the Achilefu group. It should be noted that this experiment should not be affected by uPA because there is no short spacer in this entity. The cell uptake of GNP-LSP-mCy in this 
experiment is non-specific. The cell lines were treated with mCy and GNP-LSP-mCy at the same mCy concentration. Then the cells (not the media) were imaged by the NIR fluorescence microscope (Fig. 38) and the fluorescence was quantified by a photon counter built in the microscope, at Ex/Em $780 / 830 \mathrm{~nm}, 1,3$ and $24 \mathrm{hrs}$ after the treatment (Fig. 39).

For both cell lines, and at all incubation time, the fluorescence of cell treated with GNP-LSP-mCy was significantly higher ( $>=10$ times) than that with $\mathrm{mCy}$, possibly due to both fluorescence enhancement and higher cell uptake rate of GNP-LSP-mCy than $\mathrm{mCy}$ due to the nano-size of the particle. The fluorescence of mCy or GNP-LSP-mCy in two cell lines is at a similar level, because as previously described, this product does not have a specificity.

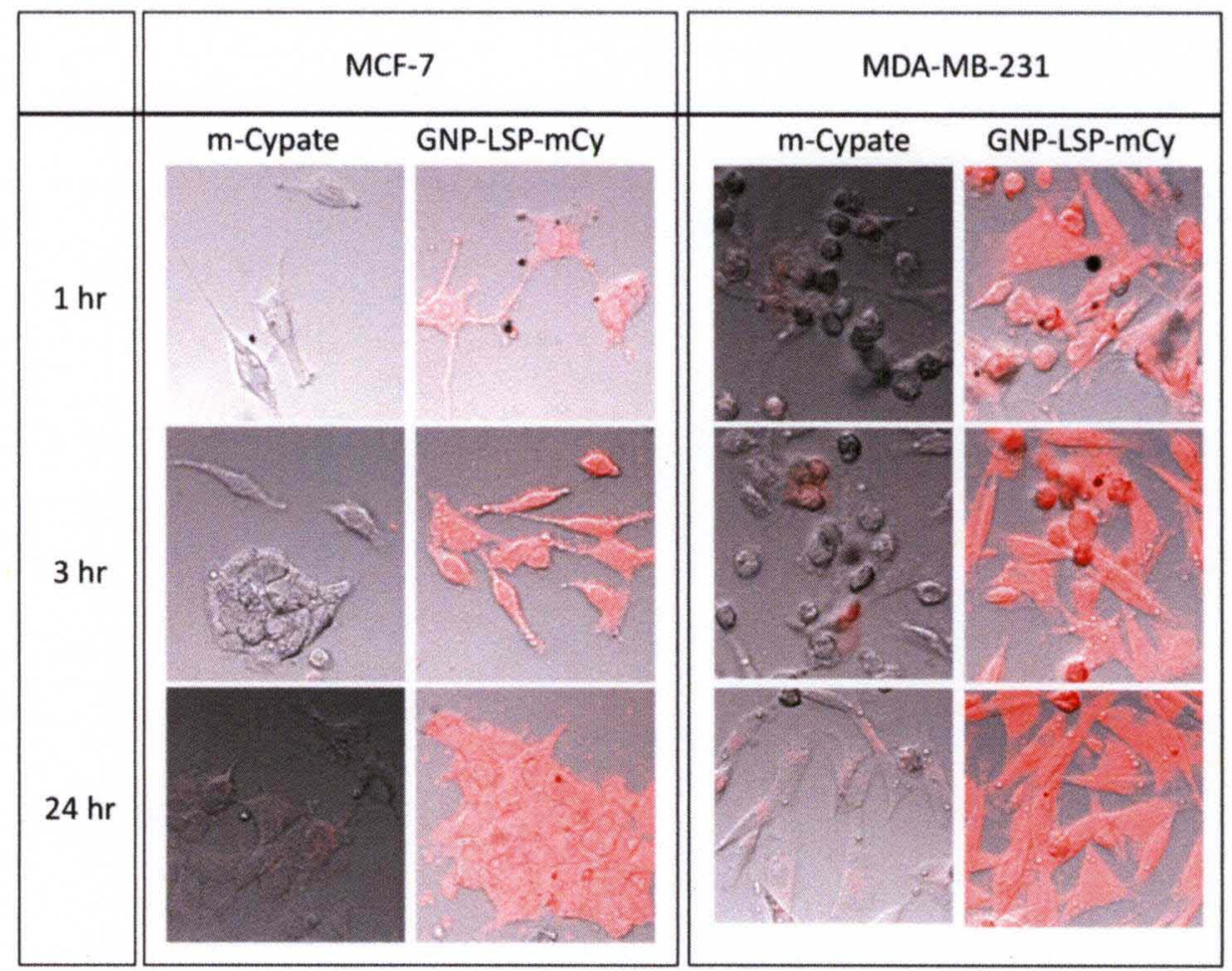

Figure 38. Fluorescence of Cypate and GNP-LSP-mCy in (a) MCF-7 (uPA-) and (b) MDA-MB-231 (uPA+) cells, 1, 3 and 24 hrs after the treatment (performed by Dr. Sharon Bloch) 

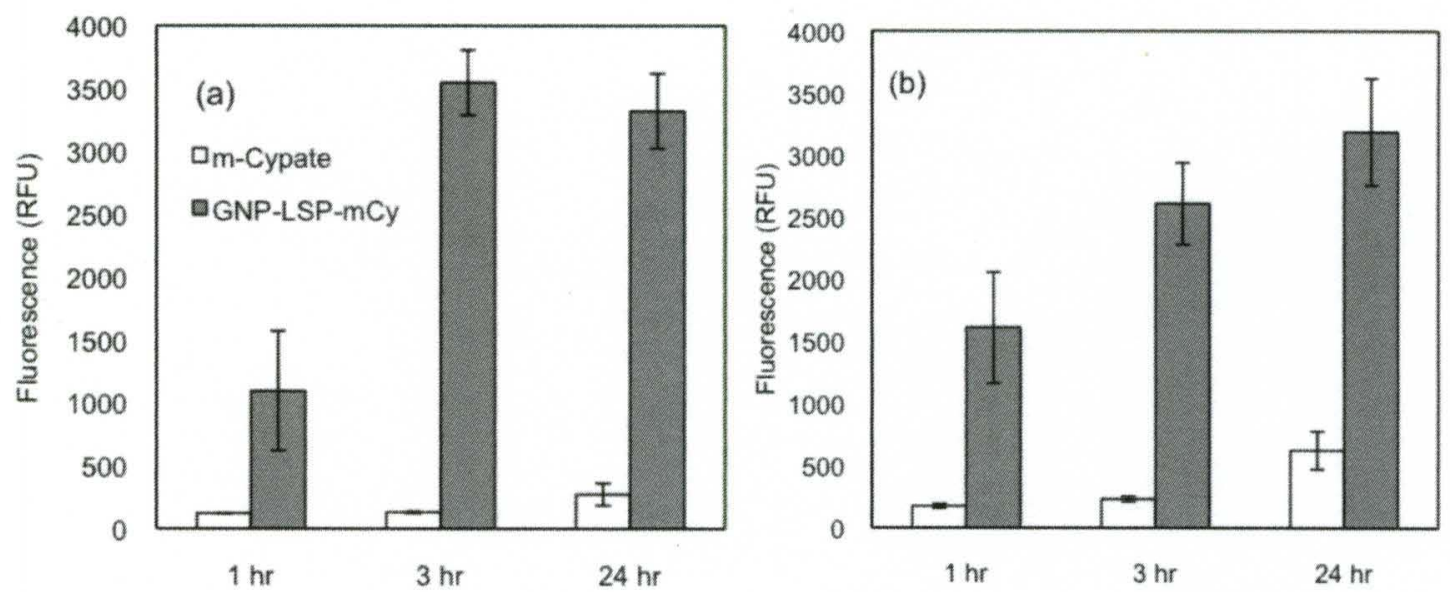

Figure 39. Fluorescence of Cypate and GNP-LSP-mCy in (a) MCF-7 (uPA-) and (b) MDA-MB-231 (uPA+) cells, 1, 3 and 24 hrs after the treatment (performed by Dr. Sharon Bloch)

In general, these results suggest that GNP-LSP-mCy can provide high contrast in the cells, by enhancing the fluorescence of Cypate or by increasing the cell uptake rate or probably by both. 


\section{CHAPTER V \\ CONCLUSIONS}

\section{A. Theoretical Analysis on Fluorescence Alteration by GNP}

A thorough analysis on the fluorescence alteration by GNP was performed for the fluorophores frequently used in the biomedical research to understand how the system parameters (the GNP size, Ex/Em wavelengths and intrinsic quantum yield of the fluorophore) and the distance between a fluorophore and a GNP affect the fluorescence output.

To acquire fluorescence quenching effect, the fluorophores with the Em wavelength near the GNP plasmon resonance peak are good candidates. For the fluorophores with Em wavelengths sufficiently longer than the GNP resonance peak (e.g., far-red or NIR fluorophores), to achieve the same level of quenching, the distance between the GNP and the fluorophore needs to be very short (e.g., $<2 \mathrm{~nm}$ ). With bigger GNPs, for the same level of quenching, the distance between the fluorophore and the GNP needs to be shorter.

The fluorescence enhancement is effective only if the Em wavelength of the fluorophore is sufficiently longer than the GNP resonance wavelength. The fluorophore with lower quantum yield have a greater potential for fluorescence enhancement. The GNP size needs to be sufficiently large to enhance fluorescence effectively. Bigger GNPs 
provide greater maximum fluorescence enhancement, and for bigger GNPs, the distance for the maximal enhancement is shorter than that for the smaller GNPs.

The theoretical study results are highly beneficial for designing fluorescence manipulation by GNPs for the fluorophores.

\section{B. Development of NIR fluorescent contrast agent for specific cancer detection}

An NIR contrast agent for highly sensitive and specific breast cancer detection was developed, utilizing both the fluorescence quenching and enhancing ability of GNPs. The fluorophore was conjugated to a GNP via two molecular spacers. One was short for the fluorescence quenching and the other was long for the maximum enhancement. The short spacer contains a G-G-R motif, uPA (enzyme secreted by breast cancer) substrate. The NIR fluorophore Cypate was used because NIR can penetrate tissue deeper than the visible light and is a derivative of the FDA-approved ICG with functional groups for conjugation. The GNP is also conjugated with targeting molecule to guide the contrast agent to the cancer site. Normally this agent is not to emit fluorescence because of the short spacer. The short spacer is cleaved when the contrast agent is delivered to the cancer site by the targeting molecule where uPA is present and the distance between the Cypate and the GNP is controlled by the long spacer length, and thus the fluorescence is emitted at an enhanced level.

The fluorescence quenching and restoration of Cypate conjugated to GNP via the short spacer was studied. For the Cypate conjugated 3.7 and $8.0 \mathrm{~nm}$ GNPs via the short spacer ( $2.5 \mathrm{~nm}$, estimated by molecular simulation), the fluorescence was quenched by 96 and $95 \%$, respectively. When uPA was applied, the fluorescence was restored. A high 
GNP concentration in the solution appeared to quench the fluorescence of the Cypate released from the GNP. For the GNP concentration of at $2.5 \mathrm{nM}$ in the solution, $74 \%$ of the fluorescence was restored.

Cypate fluorescence enhancement by GNPs at $3.7,8.0$, and $16.4 \mathrm{~nm}$ was studied using the spacers $\left[\mathrm{HS}-\left(\mathrm{CH}_{2}\right)_{\mathrm{m}} \mathrm{PEG}_{\mathrm{n}}-\mathrm{ONH}_{2}\right]$ with a length range of 3.2-4.6 $\mathrm{nm}(\mathrm{m}=12$ or 16, $\mathrm{n}=4,6$, or 8). GNPs at $3.7 \mathrm{~nm}$ appeared to be too small to enhance Cypate fluorescence. The spacer $\mathrm{HS}-\left(\mathrm{CH}_{2}\right)_{12} \mathrm{PEG}_{6}-\mathrm{ONH}_{2}(3.9 \mathrm{~nm})$ provided the highest fluorescence among the GNP sizes tested. With the GNPs of size 8.0 and $16.4 \mathrm{~nm}$, the fluorescence was enhanced up to 2.0 and 3.6 times, respectively, with the spacers tested.

Experimental study results qualitatively agreed with the theoretical estimation, although the spacer lengths estimated by molecular simulation were $1 \sim 2 \mathrm{~nm}$ longer than the distance in the theoretical result.

The performance of the novel dual spacer, containing both the short spacer section with the uPA substrate motif and the long spacer section for the maximal enhancement, was tested for the $8.0 \mathrm{~nm}$ GNP. The Cypate fluorescence was quenched by $93 \%$ when the dual spacer was conjugated with the GNP. With the uPA application, the fluorescence increased up to 1.8 times of the control.

The sell study was performed with the contrast agent with the short or long spacers using two breast cancer cell lines, MCF-7 (uPA-) and MDA-MB-231 (uPA+). For the contrast agent with the short spacer (GNP-SSP-Cy), the Cypate conjugated to $8.0 \mathrm{~nm}$ GNP via short spacer was tested. The fluorescence of GNP-SSP-Cy was expected to increase from a quenched level only in the media with uPA+ cells. However, in the uPA quantification for both cell lines, it appeared the MCF-7. The results showed that in the 
media without cells, the fluorescence of GNP-SSP-Cy was verly low ( 5 RFU). In the media with MCF-7 and MDA-MB-231 cells with the reaction time of 2 hours, the fluorescence increased to 183 and $210 \mathrm{RFU}$, respectively. The reason of the increase fluorescence in the MCF-7 cell medium might be due to small amount of uPA secretion by MCF-7 cells. For the contrast agent with long spacer, the Cypate conjugated to 8.0 nm GNP via the long spacer providing the highest enhancement was applied to MCF-7 and MDA-MB-231 cells. The fluorescence of the cells with GNP conjugated Cypate via the long spacer was 10 times higher than that of the cells with only Cypate. The higher fluorescence may be a result by both the fluorescence enhancement and by the high cell uptake rate caused by the GNP.

In terms of the utility of the study results they provide the information on how GNPs alter the fluorescence of fluorophores, which can be beneficially utilized for the fluorescence alteration in biosensing and imaging. The optical contrast agent developed here has a high sensitivity by enhanced signal. The targeting molecules and the enzymetriggered fluorescence emission offer dual specificity and also characterize the cancer type, which can be used for cancer diagnosis as well as cancer detection. This unique construct provides the biomarker-specific emission as specific as FRET or molecular beacon, but with higher sensitivity, without the restriction for DNA/RNA segments only. The design of the contrast agent can also be used in biosensing/imaging with any enzyme-substrate combination. The surface of GNPs can also be treated with cancer drugs for the cancer treatment as well as detection/diagnosis. 


\section{CHAPTER VI}

\section{FUTURE WORK}

\section{A. Completion with Targeting Molecules}

The contrast agent in this study is designed to have targeting molecule for cancerspecific delivery. A breast cancer drug Tamoxifen (Tam), and a monoclonal antibody Herceptin (Her) are currently being tested for targeting ER+ or HER2+ breast cancer, respectively. The targeting ability of the GNP-rSP-mCy with Tamoxifen (GNP-rSP$\mathrm{mCy} / \mathrm{Tam}$ )or Herceptin (GNP-rSP-mCy/Her) are being studied. The performance of the final GNP products are suggested to be tested in various breast cancer cell lines.

Tamoxifen and Herceptin have been used for breast cancer therapy. Therefore, therapeutic functions of the contrast agent are also suggested to be studied.

\section{B. Application of the Concept for Other Target Enzyme}

The contrast agent developed in this study is a model system for breast cancer imaging. In future, the enzyme-triggered fluorescence mechanism can be applied for other enzyme detection by replacing the uPA substrate motif in the short spacer to the respective substrate of the target enzyme in biosensing or bioimaging.

For biosensing which has less requirement on tissue penetration of the fluorescence or the safety of the fluorophore, many other fluorophores or quantum dots 
may be used. It is suggested that simultaneous multi-enzyme detection system be developed by using a mixture of contrast agents, in which each contrast agent is for the detection of a particular enzyme, with a fluorophore emitting fluorescence at a different wavelength from others.

\section{Development of multi-functional nano-contrast agent}

Our research group has been studying iron oxide nanoparticle-induced hyperthermia using alternating electromagnetic (AEM) field for cancer treatment for years. In the future, the contrast agent developed in this study can incorporate iron oxide nanoparticle as a core with a gold shell to provide both optical detection and non-invasive hyperthermia within one-entity. Iron oxide nanoparticle can also be used as MRI contrast agent. Gold nanoshell can be used for X-ray/CT imaging contrast agent due to increased scattering. The gold surface can be treated with targeting molecule and cancer drugs. Therefore, this multi-functional design may provide non-invasive, targeted cancer delivery and multi-modality imaging contrast for sensitive and specific cancer detection, and multi-treatment (drug and hyperthermia) within one entity. 


\section{REFERENCES}

1. Ballou B, Lagerholm BC, Ernst LA, Bruchez MP and Waggoner AS, Noninvasive Imaging of Quantum Dots in Mice, Bioconjugate Chemistry, 15, 79-86, 2004

2. Achilefu S, Jimenez HN, Dorshow RB, Bugaj JE, Webb EG, Wilhelm RR, Rajagopalan R, Johler J and Erion JL, Synthesis, in Vitro Receptor Binding, and in Vivo Evaluation of Fluorescein and Carbocyanine Peptide-Based Optical Contrast Agents, Journal of medicinal chemistry, 45, 2003-2015, 2002

3. Alexiou C, Arnold W, Klein RJ, Parak FG, Hulin P, Bergemann C, Erhardt W, Wagenpfeil S and Lübbe AS, Locoregional Cancer Treatment with Magnetic Drug Targeting 1, Cancer Research, 60, 6641, 2000

4. Alford R, Simpson HM, Duberman J, Hill GC, Ogawa M, Regino C, Kobayashi H and Choyke PL, Toxicity of Organic Fluorophores Used in Molecular Imaging: Literature Review, Molecular Imaging, 8, 341-354, 2009

5. Allen TM, Ligand-Targeted Therapeutics in Anticancer Therapy, Nature reviews. Cancer, 2, 750-763, 2002

6. Altinoglu EI and Adair JH, Near Infrared Imaging with Nanoparticles, Advanced review, 2, 461-477, 2010

7. Andreussi O, Corni S, Mennucci B and Tomasi $J$, Radiative and Nonradiative Decay Rates of a Molecule Close to a Metal Particle of Complex Shape, Journal of Chemical Physics, 121, 10190-10202, 2004

8. Anger P, Bharadwaj P and Novotny L, Enhancement and Quenching of SingleMolecule Fluorescence, Physical Review Letters, 96, 113002, 2006

9. Appenzeller T, The Man Who Dared to Think Small, Science, 254, 1300, 1991

10. Aramendia PF, Negri RM and Roman ES, Temperature Dependence of Fluorescence and Photoisomerization in Symmetric Carbocyanines. Influence of 
medium Viscosity and Molecular Structure, The Journal of Physical Chemistry, 98, 3165-3173, 1994

11. Bharadwaj P and Novotny L, Spectral Dependence of Single Molecule Fluorescence Enhancement, Optics Express, 15, 14266-14274, 2007

12. Bharadwaj $P$, Anger $P$ and Novotny L, Nanoplasmonic Enhancement of SingleMolecule Fluorescence, Nanotechnology, 18, 044017, 2007

13. Boisselier E and Astruc D, Gold Nanoparticles in Nanomedicine: Preparations, Imaging, Diagnostics, Therapies and Toxicity, Chemical Society Reviews, 38, 1759-1782, 2009

14. Bonincontro A, Francesco AD, Matzeu M, Onori $G$ and Santucci A, Conformational Changes of Lysozyme in Water-Ethanol Mixtures, Colloids and Surfaces B Biointerfaces, 10, 105-111, 1997

15. Born M and Wolf E, Principles of Optics, Cambridge University Press, London, 1999

16. Bruchez Jr M, Moronne M, Gin P, Weiss S and Alivisatos AP, Semiconductor Nanocrystals as Fluorescent Biological Labels," Science, 281, 2013-2016, 1998

17. Buschmann V, Weston $\mathrm{KD}$ and Sauer M, Spectroscopic Study and Evaluation of Red-Absorbing Fluorescent Dyes, Bioconjugate Chemistry, 14, 2003

18. Cai W, Hsu A, Li Z and Chen X, Are Quantum Dots Ready for in Vivo Imaging in Human Subjects, Nanoscale Research Letters, 2, 265-281, 2007

19. CancËs E and Mennucci B, New Applications of Integral Equations Methods for Solvation Continuum Models: Ionic Solutions and Liquid Crystals, Journal of Mathematical Chemistry, 23, 309-326, 1998

20. Cassidy $P$ and Radda G, Molecular Imaging Perspectives, Journal of the Royal Society Interface, 2, 133-144, 2005

21. Chalfie M, Tu Y, Euskirchen G, Ward W and Prasher D, Green Fluorescent Protein as a Marker for Gene Expression, Science, 263, 802-805, 1994

22. Chan $\mathrm{W}$ and Nie S, Quantum Dot Bioconjugates for Ultrasensitive Nonisotopic Detection, Science, 281, 2016-2018, 1998

23. Chen M, Yamamuro S, Farrell D and Majetich SA, Gold-Coated Iron Nanoparticles for Biomedical Applications, Journal of applied physics, 93, 7551-7554, 2003 
24. Chen SJ, Lee AF, Lee FL and Liu JH, Indocyanine Green Angiography of Central Serous Chorioretinopathy, Zhonghua Yi Xue Za Zhi (Taipei), 62, 605-613, 1999

25. Choy G, Choyke P and Libutti SK, Current Advances in Molecular Imaging: Noninvasive in Vivo Bioluminescent and Fluorescent Optical Imaging in Cancer Research, Molecular Imaging, 2, 303-312, 2003

26. Connor EE, Mwamuka J, Gole A, Murphy CJ and Wyatt MD, Gold Nanoparticles Are Taken up by Human Cells but Do Not Cause Acute Cytotoxicity, Small, 1, 325327,2005

27. Corni S and Tomasi J, Surface Enhanced Raman Scattering from a Single Molecule Adsorbed on a Metal Particle Aggregate: A Theoretical Study, Journal of Chemical Physics, 116, 1156-1165, 2002

28. Cuenca AG, Jiang H, Hochwald SN, Delano M, Cance WG and Grobmyer SR, Emerging Implications of Nanotechnology on Cancer Diagnostics and Therapeutics, Cancer, 107, 459-466, 2006

29. Dabbousi B, Rodriguez-Viejo J, Mikulec F, Heine J, Mattoussi H, Ober R, Jensen KF and Bawendi M, (Cdse)Zns Core-Shell Quantum Dots: Synthesis and Characterization of a Size Series of Highly Luminescent Nanocrystallites, The Journal of Biochemistry, Molecular Biology and Biophysics, 101, 9463-9475, 1997

30. Davis ME, Chen $Z$ and Shin DM, Nanoparticle Therapeutics: An Emerging Treatment Modality for Cancer, Nature Reviews Drug Discovery, 7, 771-782, 2008

31. Didenko VV, DNA Probes Using Fluorescence Resonance Energy Transfer (FRET): Disigns and Applications, Biotechniques, 31, 1106-1121, 2001

32. Dubertret B, Calame $M$ and Libchaber AJ, Single-Mismatch Detection Using GoldQuenched Fluorescent Oligonucleotides, Nature Biotechnology, 19, 365-370, 2001

33. Duchesne L, Gentili D, Comes-Franchini M and Fernig DG, Robust Ligand Shells for Biological Applications of Gold Nanoparticles, Langmuir, 24, 13572, 2008

34. Dulkeith E, Morteani AC, Niedereichholz T, Klar TA and Feldmann J, Fluorescence Quenching of Dye Moledules near Gold Nanoparticles: Radiative and Nonradiative Effects, Physical Review Letters, 89, 203002, 2002

35. Ekimov AI and Onushchenko AA, Quantum Size Effect in Three-Dimensional Microscopic Semiconductor Crystals, JETP Letters, 34, 345-349, 1981

36. Epstein J, Leung APK, Lee KH and Walt DR, High-Density, Microsphere-Based Fiber Optic DNA Microarrays, Biosensors Bioelectronics, 18, 541-546, 2003 
37. Evanoff DD, White RI and Chumanov G, Measuring the Distance Dependence of the Local Electromagnetic Field from Silver Nanoparticles, Journal of Physical Chemistry B, 108, 1522-1524, 2004

38. Fermi E, Nuclear Physics, University of Chicago Press, Chicago, 1950

39. Ferrari M, Cancer Nanotechnology: Opportunities and Challenges, Nature Reviews Cancer, 5, 161-171, 2005

40. Ferrucci JT and Stark DD, Iron Oxide-Enhanced MR Imaging of the Liver and Spleen: Review of the First 5 Years, AJR. American Journal of Roentgenology, $155,943-50,1990$

41. Frangioni JV, In Vivo near-Infrared Fluorescence Imaging, Current Opinion on Chemical Biology, 7, 626-634, 2003

42. Gao X, Yang L, Petros JA, Marshall FF, Simons JW and Nie S, In-Vivo Molecular and Cellular Imaging with Quantum Dots, Current Opinion in Biotechnology, 16, $63-72,2005$

43. Gerion D, Pinaud F, Williams SC, Parak WJ, Zanchet D, Weiss S and VAlivisatos PA, Synthesis and Properties of Biocompatible Water-Soluble Silica-Coated Cdse/Zns Semiconductor Quantum Dots, Journal of Physical Chemistry B, 105, 8861-8871, 2001

44. Gersten J, Spectroscopic Properties of Molecules Interacting with Small Dielectric Particles, Journal of Chemical Physics, 75, 1139-1152, 1981

45. Ghosh SK, Pal A, Kundu S, Nath S and Pal T, Fluorescence Quenching of 1Methylaminopyrene near Gold Nanoparticles: Size Regime Dependence of the Small Metallic Particles, Chemical Physics Letters, 395, 366-372, 2004

46. González-Díaz JB, García-Martín A, García-Martín JM, Cebollada A, Armelles G, Sepúlveda B, Alaverdyan $\mathrm{Y}$ and Käll M, Plasmonic $\mathrm{Au} / \mathrm{Co} / \mathrm{Au}$ Nanosandwiches with Enhanced Magneto-Optical Activity, Small, 4, 202, 2008

47. Grass RN and Stark WJ, Gas Phase Synthesis of Fcc-Cobalt Nanoparticles, Journal of Materials Chemistry, 16, 1825-1830, 2006

48. Guilbault G, Practical Fluorescence, Guilbault G, Marcel Dekker, New York, 1990

49. Hainfeld JF, Slatkin DN, Focella TM and Smilowitz HM, Gold Nanoparticles: A New X-Ray Contrast Agent, The British journal of radiology, 79, 248-253, 2006 
50. Härtling $T$, Reichenbach $P$ and Eng LM, Near-Field Coupling of a Single Fluorescent Molecule and a Spherical Gold Nanoparticle, Optics Express, 15, 12806-12817, 2007

51. Haugland RP, Yguerabide J and Stryer L, Dependence of the Kinetics of SingletSinglet Energy Transfer on Spectral Overlap, Proceedings of the National Academy of Sciences, 63, 23-30, 1969

52. Hawrysz DJ and Sevick-Muraca EM, Developments toward Diagnostic Breast Cancer Agents, Neoplasia, 2, 388-417, 2000

53. Hercules DM, Fluorescence and Phosphorescence Analysis Principles and Applications, Interscience publ., New-York; London; Sydney, 1966

54. Hergt R, Andra W, d'Ambly CG, Hilger I, Kaiser WA, Richter U and Schmidt HG, Physical Limits of Hyperthermia Using Magnetite Fine Particles IEEE Transactions on Magnetics, 34, 3745-3754, 1998

55. Hirsch LR, Stafford RJ, Bankson JA, Sershen SR, Rivera B, Price RE, Hazle JD and Halas NJ, Nanoshell-Mediated near-Infrared Thermal Therapy of Tumors under Magnetic Resonance Guidance, Proceedings of the National Academy of Sciences, $100,13549-13554,2003$

56. Hofheinz RD, Gnad-Vogt SU, Beyer U and Hochhaus A, Liposomal Encapsulated Anti-Cancer Drugs, Anti-cancer drugs, 16, 691-707, 2005

57. Honar AL and Kang KA, Effect of the Source and Detector Configuration on the Detectability of Breast Cancer, Comparative Biochemistry and Physiology - Part A: Molecular \& Integrative Physiology, 132, 9-15, 2002

58. Hong B and Kang KA, Fluorescence Enhancers for Fluorophore Mediated Biosensors for Cardiovascular Disease Diagnosis, Advances in Experimental Medicine and Biology, 578, 179-184, 2006

59. Hong B and Kang KA, Biocompatible, Nanogold-Particle Fluorescence Enhancer for Fluorophore Mediated, Optical Immunosensor, Biosensors and Bioelectronics, $21,1333-1338,2006$

60. Hong B, Tang L, Ren YJ and Kang KA, Real-Time, Automated, Fluorophore Mediated Multi-Cardiac Marker Biosensing System with Nano-Metallic Particle Reagent, Advances in Experimental Medicine and Biology, 599, 23-29, 2008

61. Hoshino A, Fujioka K, Oku T, Suga M, Sasaki YF, Ohta T, Yasuhara M, Suzuki K and Yamamoto K, Physicochemical Properties and Cellular Toxicity of Nanocrystal Quantum Dots Depend on Their Surface Modification, Nano Letters, 4, 2163-2169, 2004 
62. Huang XH, Jain PK, El-Sayed $\mathrm{IH}$ and El-Sayed MA, Determination of the Minimum Temperature Required for Selective Photothermal Destruction of Cancer Cells with the Use of Immunotargeted Gold Nanoparticles, Photochemistry and Photobiology, 82, 412-417, 2006

63. Jain PK, Huang X, El-Sayed IH and El-Sayed MA, Noble Metals on the Nanoscale: Optical and Photothermal Properties and Some Applications in Imaging, Sensing, Biology, and Medicine, Accounts of Chemical Research, 41, 1578-1586, 2008

64. Jaiswal JK and Simon SM, Potentials and Pitfalls of Fluorescent Quantum Dots for Biological Imaging, Trends in Cell Biology, 14, 497-504, 2004

65. James WD, Hirsch LR, West JL, O'Neal PD and Payne JD, Application of Inaa to the Build-up and Clearance of Gold Nanoshells in Clinical Studies in Mice, Journal of Radioanalytical and Nuclear Chemistry, 271, 455-459, 2007

66. Jin $\mathrm{H}$ and Kang KA, Application of Novel Metal Nanoparticles as Optical/Thermal Agents in Optical Mammography and Hyperthermic Treatment for Breast Cancer, Advances in Experimental Medicine and Biology, 599, 45-52, 2007

67. Johnson PB and Christy RW, Optical Constants of the Noble Metals, Physical Review Letters B, 6, 4370-4379, 1972

68. Kain S, Adams M, Kondepudi A, Yang T, Ward W and Kitts P, Green Fluorescent Protein as a Reporter of Gene Expression and Protein Localization, Biotechniques, $19,650-655,1995$

69. Kang KA, Wang J, Jasinski B and Achilefu S, Fluorescence Manipulation by Gold Nanoparticles: From Complete Quenching to Extensive Enhancement, Journal of Nanobiotechnology, 9, 16, 2011

70. Kato $\mathrm{N}$ and Caruso $\mathrm{F}$, Homogeneous, Competitive Fluorescence Quenching Immunoassay Based on Gold Nanoparticle/Polyelectrolyte Coated Latex Particles, Journal of Physical Chemistry B, 109, 19604-19612, 2005

71. Khademhosseini A and Langer R, Nanobiotechnology Drug Delivery and Tissue Engineering, Chemical Engineering Progress, 102, 38, 2006

72. Kim D, Park S, Lee JH, Jeong YY and Jon S, Antibiofouling Polymer-Coated Gold Nanoparticles as a Contrast Agent for in Vivo X-Ray Computed Tomography Imaging, Journal of the American Chemical Society, 129, 7661-7665, 2007

73. Kircher MF, Mahmood U, King RS, Weissleder R and Josephson L, A Multimodal Nanoparticle for Preoperative Magnetic Resonance Imaging and Intraoperative Optical Brain Tumor Delineation, Cancer research, 63, 8122-8125, 2003 
74. Kreibig U and Vollmer M, Optical Properties of Metal Clusters, Springer, New York, 1995

75. Kuhn S, Hakanson U, Rogobete L and Sandoghdar V, Enhancement of SingleMolecule Fluorescence Using a Gold Nanoparticle as an Optical Nanoatenna, Physical Review Letters, 97, 017402, 2006

76. Kummerlen J, Leitner A, Brunner H, Aussenegg FR and Wokaun A, Enhanced Dye Fuorescence over Silver Island Flms: Analysis of the Distance Dependence., Molecular Physics, 80, 1031-1046, 1993

77. Lakowicz JR, Radiative Decay Engineering 5: Metal-Enhanced Fluorescence and Plasmon Emission, Analytical Biochemistry, 337, 171-194, 2005

78. Lakowicz JR, Principles of Fluorescence Spectroscopy, Third Edition, Springer, New York, 2006

79. Langer R and Tirrell DA, Designing Materials for Biology and Medicine, Nature, 428, 487-492, 2004

80. Laurent $G$ and Asahi T, Enhancement of Excimer Fluorescence from Thin Dye Film by Single Gold Nanoparticles, Chemistry letters, 38, 332-333, 2009

81. Leatherdale C, Woo W-K, Mikulec F and Bawendi M, On the Absorption Cross Section of the Nanocrystal Quantum Dots, The Journal of Biochemistry, Molecular Biology and Biophysics, 106, 7619-7622, 2002

82. Li JJ and Tan W, A Real-Time Assay for DNA Sticky-End Pairing Using Molecular Beacons, Analytical Biochemistry, 312, 251-254, 2003

83. Libutti SK, Giulio F, Paciotti AA, Byrnes H, Richard A, William E, Gannon Jr. M, Walker GD, Seidel NY and Lawrence T, Phase I and Pharmacokinetic Studies of Cyt-6091, a Novel Pegylated Colloidal Gold-Rhtnf Nanomedicine, Clinical Cancer Research, 16, 6139-6149, 2010

84. Lim Y, Kim S, Nakayama A, Stott N, Bawendi M and Frangioni J, Selection of Quantum Dot Wavelengths for Biomedical Assays and Imaging., Molecular Imaging, 2, 50-64, 2003

85. Loo C, Lowery A, Halas NJ, West J and Drezeck J, Immunotargeted Nanoshells for Integrated Cancer Imaging and Therapy, Nano Letters, 5, 709-711, 2005

86. Lou HJ and Tan W, Femtoliter Microarray Wells for Ultrasensitive DNA/mRNA Detection, Instrumentation Science and Technology, 30, 465-476, 2002 
87. Love JC, Estroff LA, Kriebel JK, Nuzzo RG and Whitesides GM, Self-Assembled Monolayers of Thiolates on Metals as a Form of Nanotechnology, Chemical Reviews, 105, 1103-1169, 2005

88. Lübbe AS, Bergemann C, Riess H, Schriever F, Reichardt P, Possinger K, Matthias M, Dörken B, Herrmann F, Gürtler R, Hohenberger P, Haas N, Sohr R, Sander B, Lemke AJ, Ohlendorf D, Huhnt $W$ and Huhn D, Clinical Experiences with Magnetic Drug Targeting: A Phase I Study with 4'-Epidoxorubicin in 14 Patients with Advanced Solid Tumors, Cancer Research, 56, 4686, 1996

89. Mankoff DA, Molecular Imaging as a Tool for Translating Breast Cancer Science, Breast Cancer Research, 10, 12-19, 2008

90. Marshall MV, Rasmussen JC, Tan IC, Aldrich MB, Adams KE, Wang X, Fife CE, Maus EA, Smith LA and Sevick-Muraca EM, Near-Infrared Fluorescence Imaging in Humans with Indocyanine Green: A Review and Update, The Open Surgical Oncology Journal, 2, 12-25, 2010

91. Mazumder S, Dey R, Mitra MK, Mukherjee S and Das GC, Review: Biofunctionalized Quantum Dots in Biology and Medicine, Journal of Nanomaterials, 2009, 815734, 2009

92. Medintz IL, Uyeda HT, Goldman ER and Mattoussi H, Quantum Dot Bioconjugates for Imaging, Labelling and Sensing, Nature Materials, 4, 435-446, 2005

93. Meng X, Seton HC, Lu LT, Prior IA, Thanh NTK and Song B, Magnetic Copt Nanoparticles as Mri Contrast Agent for Transplanted Neural Stem Cells Detection, Nanoscale, 3, 977-984, 2011

94. Mennucci B, Cances E and Tomasi J, Evaluation of Solvent Effects in Isotropic and Anisotropic Dielectrics and in Ionic Solutions with a Unified Integral Equation Method: Theoretical Bases, Computational Implementation, and Numerical Applications, Journal of Physical Chemistry B, 101, 10506-10517, 1997

95. Merchant BG, Gold, the Noble Metal and the Paradoxes of Its Toxicology., Biologicals, 26, 49-59, 1998

96. Michalet X, Pinaud F, Lacoste TD, Dahan M, Bruchez M, Alivisatos PA and Weiss S, Properties of Fluorescent Semiconductor Nanocrystals and Their Application to Biological Labeling, Single Molecules, 2, 261-76, 2001

97. Mie G, Beiträge Zur Optik Trüber Medien, Speziell Kolloidaler Metallösungen, Annals of Physics, 330, 337-445, 1908 
98. Miyawaki A, Sawano A and Kogure T, Lighting up Cells: Labelling Proteins with Fluorophores, Nature Cell Biology, Sep, S1-7, 2003

99. Murray CB, Norris DJ and Bawendi M, Synthesis and Characterization of Nearly Monodisperse Cde $(\mathrm{E}=\mathrm{S}, \mathrm{Se}, \mathrm{Te})$ Semiconductor Nanocrystallites, Journal of the American Chemical Society, 115, 8706-8715, 1993

100. Nakamura $\mathrm{T}$ and Hayashi $\mathrm{S}$, Enhancement of Dye Fluorescence by Gold Nanoparticles: Analysis of Particle Size Dependence, Japan Journal of Applied Physics, 44, 6833-6837, 2005

101. Neeves AE and Birnboim MH, Composite Structures for the Enhancement of Nonlinear-Optical Susceptibility, Journal of the Optical Society of America B, 6, 787-796, 1989

102. Ntziachristos V and Chance B, Probing Physiology and Molecular Function Using Optical Imaging: Applications to Breast Cancer" Breast Cancer Research, 3, 41-46, 2001

103. O'Reilly JE, Fluorescence Experiments with Quinine, Journal of chemistry education, 52, 610, 1975

104. O'Neal DP, Hirsch LR, Halas NJ, Payne JD and West JL, Photo-Thermal Tumor Ablation in Mice Using near Infrared-Absorbing Nanoparticles, Cancer Letters, 209, 171-176, 2004

105. Ostuni E, Chapman RG, Michael N. Liang, Gloria Meluleni, Gerald Pier, Donald E. Ingber and Whitesides GM, Self-Assembled Monolayers That Resist the Adsorption of Proteins and the Adhesion of Bacterial and Mammalian Cells, Langmuir, 17, 6336-6343, 2001

106. Paciotti GF, Kingston DGI and Tamarkin L, Colloidal Gold: A Novel Nanoparticle Vector for Tumor Directed Drug Delivery, Drug Delivery, 11, 169-183, 2004

107. Paciotti GF, Kingston DGI and Tamarkin L, Colloidal Gold Nanoparticles: A Novel Nanoparticle Platform for Developing Multifunctional Tumor-Targeted Drug Delivery Vectors, Drug Development Research, 67, 47-54, 2006

108. Papahadjopoulos D, Allen TM, Gabizon A, Mayhew E, Matthay K, Huang K, Lee KD, Woodle MC, Lasic D, Redemann C and Martin FJ, Sterically Stabilized Liposomes: Improvements in Pharmacokonetics and Antitumor Therapeutic Efficacy, Proceedings of the National Academy of Sciences of the United States of America, 88, 11460-11464, 1991

109. Park JW, Liposome-Based Drug Delivery in Breast Cancer Treatment, Breast Cancer Research, 4, 95-99, 2002 
110. Patterson G, Day RN and Piston D, Fluorescent Protein Spectra, Journal of Cell Science, 114, 837-838, 2001

111. Piston DW and Kremers G-J, Fluorescent Protein Fret: The Good, the Bad and the Ugly, Trends in Biochemical Sciences, 32, 407-414, 2007

112. Prasher DC, Eckenrode VK, Ward WW, Prendergast FG and Cormier MJ, Primary Structure of the Aequorea Victoria Green-Fluorescent Protein, Gene, 111, 229-233, 1992

113. Raabe A, Beck J, Gerlach R, Zimmermann $M$ and Seifert V, Near-Infrared Indocyanine Green Video Angiography: A New Method for Intraoperative Assessment of Vascular Flow, Neurosurgery, 52, 132-139, 2003

114. Reed M, Randall J, Aggarwal R, Matyi R, Moore T and Wetsel A, Observation of Discrete Electronic States in a Zero-Dimensional Semiconductor Nanostructure, Physical Review Letters, 60, 535-537, 1988

115. Rendell D, Fluorescence and Phosphorescence (Analytical Chemistry by Open Learning), John Wiley, Chichester, 1987

116. Resch-Genger U, Grabolle M, Cavaliere-Jaricot S, Nitschke R and Nann T, Quantum Dots Versus Organic Dyes as Fluorescent Labels, Nature Methods, 5, 763-775, 2008

117. Rietdorf J, Microscopy Techniques, Springer-Verlag GmbH, Berlin Heidelberg, 2005

118. Ritchie RH, Plasma Losses by Fast Electrons in Thin Films, Physical Review, 106, $874-881,1957$

119. Rodriguez J, Scherlis D, Estrin D, Aramendia PF and Negri RM, Aml Study of the Ground and Excited State Potential Energy Surfaces of Symmetric Carbocyanines, Journal of Physical Chemistry A, 101, 6998-7006, 1997

120. Root SW, Andrews GA, Kniseley RM and Tyor MP, The Distribution and Radiation Effects of Intravenously Administered Colloidal Gold-198 in Man, Cancer, 7, 856-866, 1954

121. Rossetti R, Nakahara S and Brus LE, Quantum Size Effects in the Redox Potentials, Resonance Raman Spectra, and Electronic Spectra of Cds Crystallites in Aqueous Solution, Journal of Chemical Physics, 79, 1086, 1983

122. Ruckebusch C, Nedjar-Arroume N, Magazzeni S, Huvenne J-P and Legrand P, Hydrolysis of Haemoglobin Surveyed by Infrared Spectroscopy: I. Solvent Effect 
on the Secondary Structure of Haemoglobin, Journal of Molecular Structure, 478, 185,1999

123. Ryman-Rasmussen JP, Riviere JE and Monteiro-Riviere NA, Surface Coatings Determine Cytotoxicity and Irritation Potential of Quantum Dot Nanoparticles in Epidermal Keratinocytes, Journal of Investigative Dermatology, 127, 143-153, 2007

124. Salata OV, Applications of Nanoparticles in Biology and Medicine, Journal of Nanobiotechnology, 2, 3-9, 2004

125. Sakatani K, Kashiwasake-Jibu M, Taka Y, Wang S, Zuo H, Yamamoto K and Shimizu K, Noninvasive Optical Imaging of the Subarachnoid Space and Cerebrospinal Fluid Pathways Based on Near-Infrared Fluorescence, Journal of Neurosurgery, 87, 738-745, 1997

126. Scaffardi L, Di Paolo RE and Duchowicz R, Simultaneous Absorption and Fluorescence Analysis of the Cyanine Dye Doci, Journal of Photochemistry and Photobiology A, 107, 185, 1997

127. Schneider G, Decher G, Nerambourg N, Praho R, Werts MHV and BlanchardDesce M, Distance-Dependent Fluorescence Quenching on Gold Nanoparticles Ensheathed with Layer-by-Layer Assembled Polyelectrolytes, Nano Letters, 6, 530536,2006

128. Shukla R, Bansal V, Chaudhary M, Basu A, Bhonde R and Sastry M, Biocompatibility of Gold Nanoparticles and Their Endocytotic Fate inside the Cellular Compartment: A Microscopic Overview, Langmuir, 21, 10644-10654, 2005

129. Singh R and Lillard JW, Nanoparticle-Based Targeted Drug Delivery, Experimental and Molecular Pathology, 102, 38, 2009

130. Skala MC, Crow MJ, Wax A and Izatt JA, Photothermal Optical Coherence Tomography of Epidermal Growth Factor Receptor in Live Cells Using Immunotargeted Gold Nanospheres, Nano Letters, 8, 3461-3467, 2008

131. Smithpeter C, Dunn A, Drezek R, Collier T and R. R-K, Near Real Time Confocal Microscopy of Cultured Amelanotic Cells: Sources of Signal, Contrast Agents and Limits of Contrast, Journal of Biomedical Optics, 3, 429-436, 1998

132. Sperling RA, Rivera-Gil P, Zhang F, Zanella $M$ and Parak WJ, Biological Applications of Gold Nanoparticles, Chemical Society Reviews, 37, 1896-1908, 2008 
133. Spiker JO, Kang KA, Drohan W and Bruley DF, Protein C Detection Via Fluorophore Mediated Immuno-Optical Biosensor, Advances in experimental medicine and biology, 428, 621, 1998

134. Stryer L and Haugland R, Energy Transfer: A Spectroscopic Ruler, Proceedings of the National Academy of Sciences, 58, 719-726, 1967

135. Tan W, Wang K and Drake TJ, Molecular Beacons, Current Opinion on Chemical Biology, 8, 547-553, 2004

136. Taton TA, Nanostructures as Tailored Biological Probes, Trends in Biotechnology, $20,277-279,2002$

137. Tomasi J, Mennucci B and Cammi R, Quantum Mechanical Continuum Solvation Models, Chemical Reviews, 105, 2999, 2005

138. Tyagi S and Kramer FR, Molecular Beacons: Probes That Fluoresce Upon Hybridization, Nature Biotechnology, 14, 303-308, 1996

139. Vet JAM, Van der Rijt BJM and Blom H, Molecular Beacons: Colorful Analysis of Nucleic Acids, Expert Review of Molecular Diagnostics, 2, 77-86, 2002

140. Vulkovic S, Corni S and Mennucci B, Fluorescence Enhancement of Chromophore Close to Metal Nanoparticle - Optimal Setup Revealed by the Polzrizable Continuu Model, Journal of Physical Chemistry C, 113, 121-133, 2009

141. Waggoner A, Fluorescent Labels for Proteomics and Genomics, Current Opinion on Chemical Biology, 10, 62-66, 2006

142. Wang C, Shim M and Guyot-Sionnest P, Electrochromic Nanocrystal Quantum Dots, Science, 291, 2390-2392, 2001

143. Wang J, Nantz M, Achilefu S and Kang KA, Fret-Like Fluorophore-Nanoparticle Complex for Highly Specific Cancer Localization, Advances in Experimental Medicine and Biology, 662, 407-414, 2010

144. Wang J, Nantz M, Achilefu S and Kang KA, Gold Nanoparticle-Fluorophore Complex for Conditionally Fluorescing Signal Mediator, Analytica Chimica ACTA, 696, 96-104, 2011

145. Wang J, O'Toole M, Massey A, Biswas S, Nantz M, Achilefu S and Kang KA, Highly Specific, NIR Fluorescent Contrast Agent with Emission Controlled by Gold Nanoparticle, Advances in Experimental Medicine and Biology, 701, 149-54, 2011 
146. Wang J, Jiang S, Li Z, diFlorio-Alexander R, Barth R, Kaufman P, Pogue B and Paulsen K, In Vivo Quantitative Imaging of Normal and Cancerous Breast Tissue Using Broadband Diffuse Optical Tomography, Medical Physics, 37, 3715-3724, 2010

147. Weissleder R, Elizondo G, Wittenberg J, Rabito CA, Bengele HH and Josephson L, Ultrasmall Superparamagnetic Iron Oxide: Characterization of a New Class of Contrast Agents for Mr Imaging, Radiology, 175, 489-493, 1990

148. West J and Halas N, Engineered Nanomaterials for Biophotonics Applications: Improving Sensing, Imaging, and Therapeutics, Annual Review of Biomedical Engineering, 5, 285-292, 2003

149. Whitesides GM, The "Right" Size in Nanobiotechnology, Nature Biotechnology, $21,1161-1165,2003$

150. Wokaun A, Lutz HP, King AP, Wild UP and Ernst RR, Energy Transfer in Surface Enhanced Luminescence, Journal of Chemical Physics, 79, 1, 1983

151. Yelin D, Oron D, Thiberge S, Moses E and Silberberg Y, Multiphoton PlasmonResonance Microscopy, Optics Express, 11, 1385-1391, 2003

152. Zayats AV, Smolyaninov II and Maradudin AA, Nano-Optics of Surface Plasmon Polaritons, Physics Reports-Review Section of Physics Letters, 408, 131-314, 2005

153. Zhang J, Campbell RE, Ting AY and Tsien RY, Creating New Fluorescent Probes for Cell Biology, Nature Review Molecular Cell Biology, 3, 906-918, 2002

154.Zharov VP, Galitovskaya EN, Johnson C and Kelly T, Synergistic Enhancement of Selective Nanophotothermolysis with Gold Nanoclusters: Potential for Cancer Therapy, Lasers in Surgery and Medicine, 37, 219-226, 2005 


\section{APPENDIX I \\ GOLD NANOPARTICLES FOR SENSITIVITY ENHANCEMENT IN REAL-TIME, IMMUNO- OPTICAL CARDIAC MARKER SENSING}

\section{A. BACKGROUND}

\section{Four Cardiac-marker Sensing System for AMI Diagnosis}

Acute myocardial infarction (AMI) is the world's leading cause of morbidity and mortality. It is also a disease with a high rate of misdiagnosis due to the low sensitivity of the current diagnostic tools used in the emergency room [Wu, et al., 1999; Pope, et al., 2000]. A better method for AMI diagnosis is to quantify the elevation of cardiac markers in blood plasma [Bernard, et al., 1979]. Currently, the cardiac markers are usually quantified in a central laboratory, and it often takes hours from when it is ordered till the results are received. Due to the needs for urgent treatment of the disease nature, a rapid and accurate point-of-care (POC) system is critical. The fiber-optic, fluorophoremediated, immuno-sensing system has demonstrated accurate, sensitive, rapid, and reliable simultaneous quantification of various biomarkers [Wu, et al., 1999; Pope, et al., 2000]. The Kang group has been developing portable, near-infrared, real-time multi cardiac marker sensing system by immuno-optical sinsing. The four markers selected for our sensing system were as follows: B-type natriuretic peptide (BNP) and C-reactive protein (CRP) are crucial markers for the diagnosis of congestive heart failure and acute 
coronary syndromes [Maisel, et al., 2002; Sabatine, et al., 2002]; Myoglobin (MG) and cardiac troponin I (cTnI) are important markers for early diagnosis of a heart attack [Apple, et al., 1999]. Our research group has previously determined the clinically important sensing range of the marker to be $26 \sim 260$ pM for BNP; 30 300 pM for cTnI; 4 40 nM for $\mathrm{MG}$ and 5.6 56 nM for CRP [Tang, et al., 2005; Tang and Kang, 2006; Tang et al., 2006], b0ased on the assessment of the clinical results. For BNP and cTnI, their concentrations in plasma at an early disease stage are only tens of pico molar level, requiring extremely high sensitivity, which can be improved by enhancing the signal.

\section{GNP Reagent for Fluorescence Enhancement}

The Kang group has been studying to use GNPs in the fluorophore mediated, fiber-optic biosensing to enhance the fluorescence to improve the sensitivity of the sensor [Hong and Kang, 2006; Kang and Hong, 2006]. The sensor performs a fluorophoremediated sandwich immunoassay on the sensor surface, and the fluorescence intensity is correlated with the analyte concentration in blood plasma [Kang et al., 1997; Spiker et al., 1998; Kwon et al., 2002; Baler et al., 2002].

In an attempt to enhance the sensitivity of the sensing system GNPs were applied to the sensor surface after the sandwich formation, and the fluorescence intensity was observed and compared with that without GNP application. The minimum distance between the GNP and the fluorophore was adjusted by a self-assembled monolayer (SAM) on the GNP surface. The effect of GNP size and SAM thickness was studied. Among the GNP-SAMs tested (GNP size of 2, 5, and $10 \mathrm{~nm}$ and SAM thickness of 1, 2 and $3 \mathrm{~nm}$ ), $5 \mathrm{~nm}$ GNPs coated with $2 \mathrm{~nm}$ SAMs (5 nm GNP-SAM2 $\mathrm{nm}$ ) were found to 
be the best enhancer, and the enhancement level was up to $230 \%$.

Hong and Kang [2006] also found that some solvents could also enhance the fluorescence of a fluorophore without denaturing the antibody on the sensor surface, by shifting the fluorophore excitation/emission wavelengths, and/or by changing the transcis isomerization direction, and/or by shrinking fluorophores tagged proteins, so that the fiber received more fluorescence. They have tested various solvents and found that 1butanol and ethanol showed the highest and $2^{\text {nd }}$ highest enhancement, respectively.

To maximize the enhancement effect, Hong and Kang [2006] mixed the two enhancers, the GNP-SAM and the solvent, to form GNP reagent (GNPR). They have tested the GNPR of $5 \mathrm{nmGNP}-\mathrm{SAM} 2 \mathrm{~nm}$ in ethanol with Alexa fluorophore (Alex-647) for four-cardiac marker sensing and achieved sensitivity improvement by $1.5 \sim 3$ times.

In this study, our effort was focused on further improving the sensitivity by using GNPR with butanol. Improving sensitivity allows reducing of the sensor size and, therefore, the sample and reagents volume (i.e., assay cost reduction), and also portability.

\section{B. MATERIALS, INSTRUMENTS AND METHODS}

\section{Sensor Preparation}

For the cardiac markers and their respective monoclonal antibodies, BNP was purchased from Bachem (Torrance, $\mathrm{CA}$ ) and monoclonal IgG against human BNP was from Strategic Biosolutions (Newark, DE). cTnI, MG, and CRP, and their antibodies were obtained from Fitzgerald Industries (Concord, MA). 
Four cardiac marker biosensors were constructed, following the protocol established by Tang et al. [2006]. Briefly the surface of four quarts fibers (Research International, Inc.; Monroe, WA) were treated and immobilized with streptavidin (Sigma/Aldrich, St. Louis, MO). 0.1 M ethanolamine (Sigma/Aldrich) was applied to terminate the reactive function groups on the sensor surface. The monoclonal antibodies $\left(1^{\circ} \mathrm{Mab}\right)$ against the respective markers were conjugated with biotin (Sigma/Aldrich) and immobilized on the surface of fiber via streptavidin-biotin bond, and the sensors were encased in a micro-sensing chamber. The fluorophore Alexa Fluor ${ }^{\circledR} 647$ (AF647; max. excitation/emission wavelengths, 649/666 nm) was from Invitrogen (Carlsbad, CA). The respective second monoclonal antibodies $\left(2^{\circ} \mathrm{Mab}\right)$ were conjugated with AF647 following the manufacture's instruction.

\section{Assay Protocol with the Sensor}

The emulated plasma was prepared $103 \mathrm{mg} / \mathrm{ml}$ human serum albumin (HSA; Sigma/Aldrich) in the PBS buffered solution, because it provides similar physical properties for sensing [Hong and Kang, 2006]. Samples with cardiac markers were prepared by adding known amount of cardiac markers to the emulated plasma.

The cardiac marker assay was proceeded as follows: a sample was introduced into the sensing chamber and incubated for $3 \mathrm{~min}$ with the sample circulating at a rate of 1.2 $\mathrm{cm} / \mathrm{sec}$. The sensor was then washed with phosphate buffered saline with $0.1 \%$ Tween 20 (PBST, $\mathrm{pH} 7.4$ ) for 1 minute in circulation to remove the unbound molecules in the sample. Then the baseline fluorescence was measured using the fluorometer (Analyte $2000^{\mathrm{TM}}$; Research International). The instrument has four sensing ports. AF647 tagged $2^{\circ} \mathrm{Mab}$, at a concentration of $10 \mu \mathrm{g} / \mathrm{ml}$, was then applied to the chamber and incubated for 
4 minutes the reagent in the circulation mode to react with the bound analyte. For the simultaneous four marker sensing, all four $2^{\circ}$ Mabs were mixed and applied to the sensing system. The sensor was washed with PBST for 1 minute and the second fluorescence readings were taken. The signal difference between this signal and the baseline subtracted by the negative control is the signal intensity of the sample.

For preparation of the GNPR, 5nm-GNPs coated with citric acid were purchased from Ted Pella (Redding, CA). 1-butanol was purchased from Sigma/Aldrich. For the sensing with a GNPR. When GNPR was used in sensing, there were slight changes in the sensing protocol: For the baseline measurement, the GNPR was applied before the sample incubation. For the signal by the sample, GNPRs were applied after the second antibody was reacted with the analyte on the sensor surface. The difference between the latter and the former was taken as the signal with GNPR. The enhancement was defined as the fluorescence signal with the GNPR divided by the fluorescence from the sensor without GNPR.

\section{Microfluidic System and Automated Sensing Operation}

The sensing chip and microchannel network of the sensing unit were microfabricated by the Chong H. Ahn group [Sohn, et al.,2005 and Hong, et al., 2008]. The system includes a polycarbonate mother board with imbedded network of the microchannels; a four-channel, plastic sensing module; a micro-solenoid pump (12 v, 50 $\mu \mathrm{L}$ per stroke, $2 \mathrm{~W}$ ); seven micro-solenoid valves (12 v, $280 \mathrm{~mW}$, Lee Co.; Westbrook, CT); a data acquisition (DAQ) card (USB-6008, 8 inputs, 12 bits, 10000 samples/s, multifunctional I/O, National Instruments; Austin, TX); and a drive circuit with a power plug, a power switch, and a power LED. 


\section{RESULTS AND DISCUSSION}

\section{Reduction in Sensor Size}

Here, we have attempted to reduce the sensor size from 3 to $1.5 \mathrm{~cm}$ by using 5 nmGNP-SAM2 $\mathrm{nm}$ in butanol. Since BNP has the lowest sensing range among the four cardiac markers, BNP sensor size was our main concern.

Figure API-1 shows the performance of the BNP sensors with the sensor size of (a) 3 and (b) $1.5 \mathrm{~cm}$, with and without GNPR, when the total sensing time was $15 \mathrm{~min}$. In terms of the signal intensity enhancement by GNPR, the signals were enhanced by 4 and 3 times for the $3 \mathrm{~cm}$ and $1.5 \mathrm{~cm}$ sensors, respectively. For the $1.5 \mathrm{~cm}$, the signal without GNPR was very low in the entire sensing range. The signal for the $1.5 \mathrm{~cm}$ sensor with GNPR was approximately $40 \%$ of that of the $3 \mathrm{~cm}$ sensor. Nonetheless, the correlation coefficient was 0.96 ( 0.95 for $3 \mathrm{~cm}$ sensor), and the signal to noise ratio ( $\mathrm{S} / \mathrm{N})$ was 4.1 ( 3.8 for $3 \mathrm{~cm}$ sensor), showing that the performance of the $1.5 \mathrm{~cm}$ sensor was still satisfactory, but with less sample and reagent.

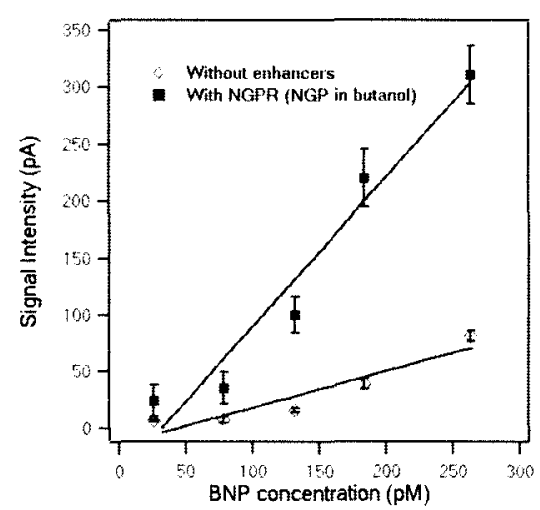

(a)

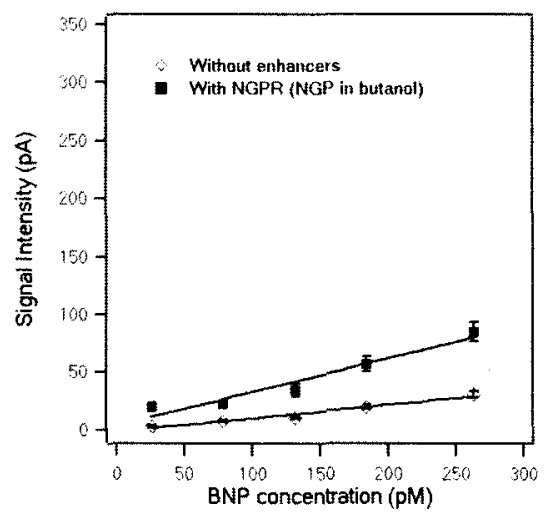

(b)

Figure API-1. Performance of BNP sensor at sensor sizes of (a) $3 \mathrm{~cm}$ and (b) $1.5 \mathrm{~cm}$ with and without GNPR ( $5 \mathrm{nmGNP}-\mathrm{SAM} 2 \mathrm{~nm}$ in butanol). The total sensing time, $15 \mathrm{~min}$ 


\section{Four Cardiac Marker Sensing by the Mini-sensor}

Since the sensing performance of the $1.5 \mathrm{~cm}$ BNP sensor was satisfactory, the 1.5 $\mathrm{cm}$ sensor size was then tested for all four cardiac markers in the four sensor micro sensing chip. The size of the sensing chip for four cardiac marker was reduced from $4 \times 4$ $\mathrm{cm}$ (for $3 \mathrm{~cm}$ sensors) to $2 \times 2.5 \mathrm{~cm}$ (for $1.5 \mathrm{~cm}$ sensors) accordingly. A schematic diagram of the chip is shown in Fig. API-2. As previously shown by Hong, et al., [2008], on the inner surface of the microchannel, bumps/baffles were microfabricated to create local turbulence, to facilitate the analyte transport to the sensor surface. The minisensing chip requires a sample size of only $200 \mu \mathrm{l}$, and therefore, only $0.5 \mathrm{ml}$ of a blood sample is needed for an assay.

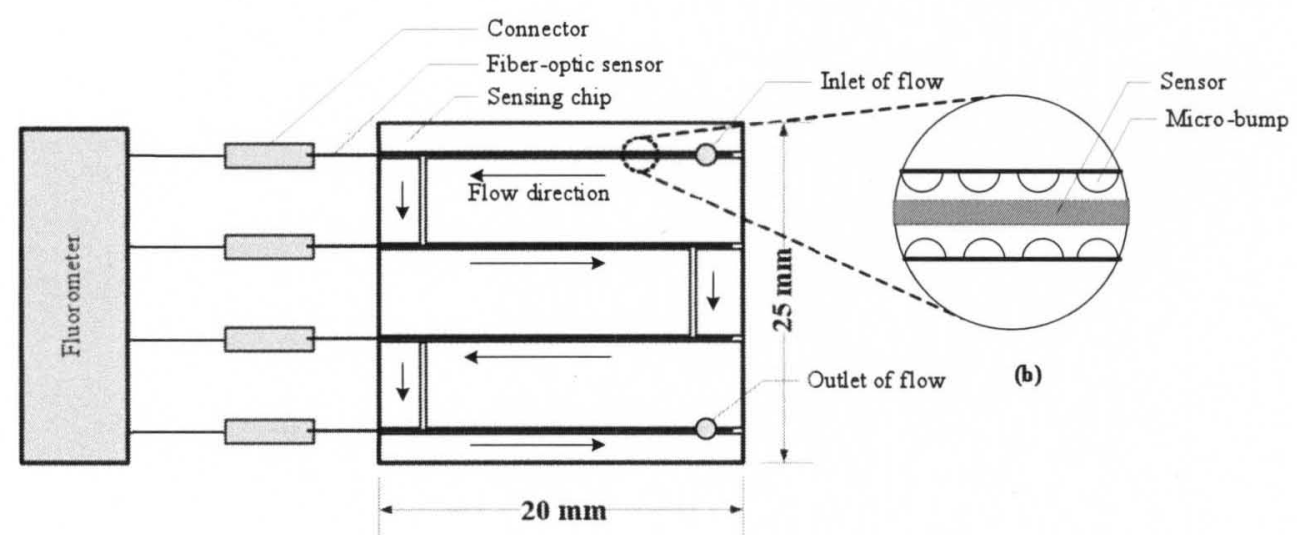

(a)

Figure API-2. (a) Schematic diagram of mini-sensing chip and (b) Enlarged diagram of a sensing chamber

Figure API-3 shows the performance of the four-cardiac marker sensors with and without GNPR, when the entire senisng time was $15 \mathrm{~min}$. For BNP and cTnI, the signal range without using GNPR was only $3 \sim 30 \mathrm{pA}$. With GNPR, the signal was enhanced by 3 and 4 times, respectively. The $\mathrm{S} / \mathrm{N}$ ratios were 4.1 and 4.5 , and the correlation 
coefficients were 0.96 and 0.97 , respectively. For MG and CRP, the signal ranges without and with enhancer were $4 \sim 105 \mathrm{pA}$ and $90 \sim 400 \mathrm{pA}$, respectively. The signals were enhanced by 5 and 2 times and the $\mathrm{S} / \mathrm{N}$ ratios were 5.2 and 6.7 for MG and $\mathrm{CRP}$, respectively. As can be seen from these values, these two markers do not need enhancer. But, since the four marker sensing needs to be done simultaneously with a single sample and reagents, GNPR was applied for all four sensors.

All four $1.5 \mathrm{~cm}$ sensors showed linear relationships between the marker concentration and the signal intensity, with correlation coefficients above 0.95 .

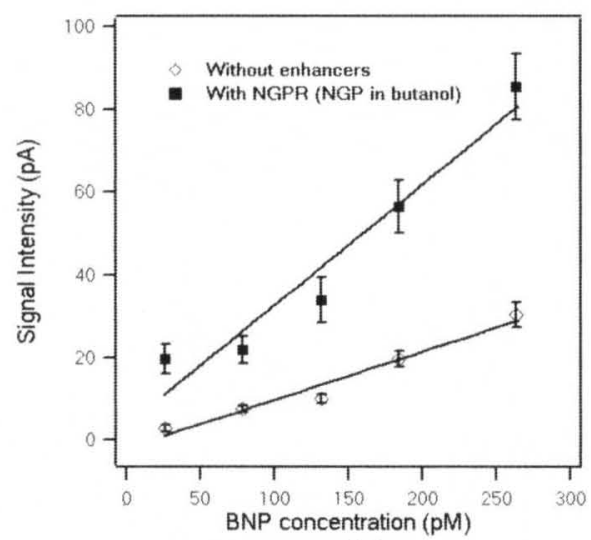

(a)

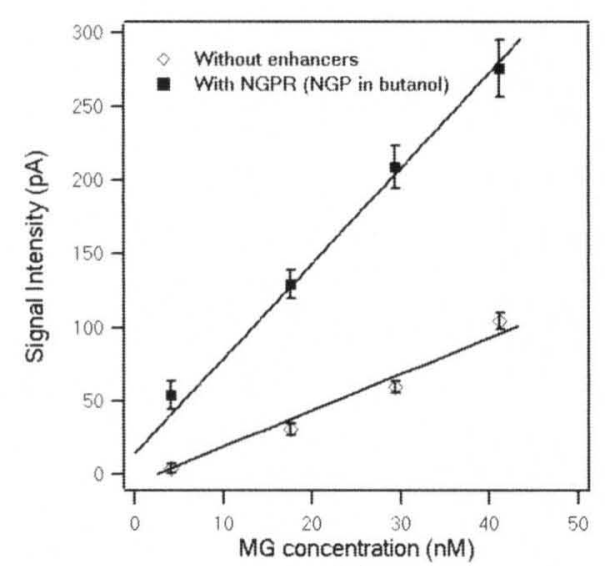

(c)

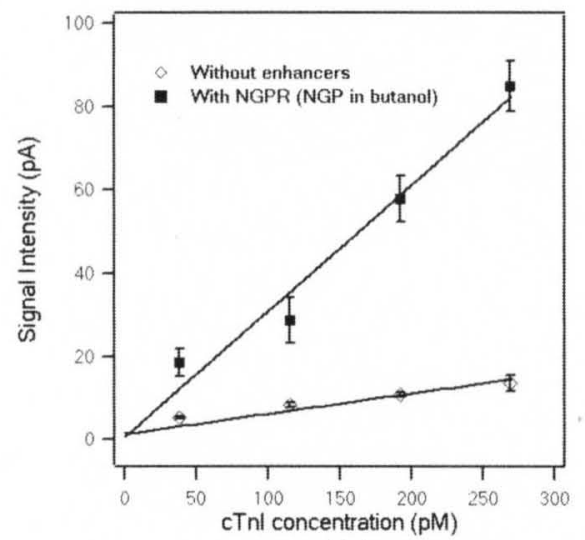

(b)

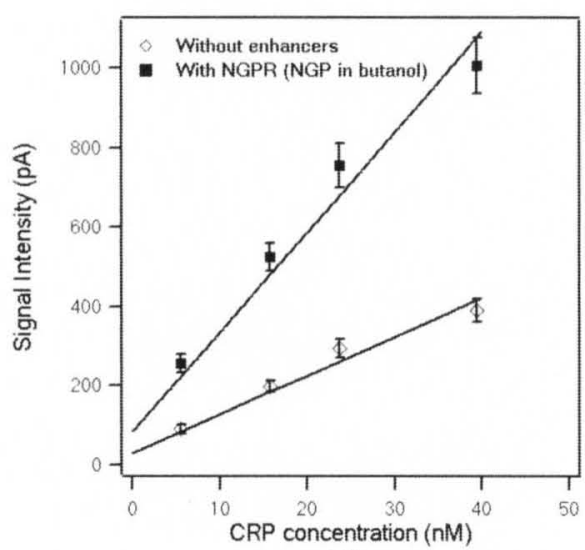

(d)

Figure API-3. Sensing performance of $1.5 \mathrm{~cm}$ (a) BNP, (b) cTnI, (c) MG, and (d) CRP sensors, with and without GNPR (5 nm GNP-SAM2 $\mathrm{nm}$ in butanol) 


\section{CONCLUSIONS AND FUTURISTIC, PORTABLE ALL-IN-ONE DEVICE}

Our MEMS based sensing device with mini-sensing chips $(2 \times 2.5 \mathrm{~cm})$ and novel gold nanoparticle reagent can accurately quantify four cardiac markers simultaneously at clinically significant concentration ranges within $15 \mathrm{~min}$, requiring a plasma sample volume of only $200 \mu$ l. The entire sensing system may be converted to be a highly portable, all-in-one, point-of-care (POC) sensing device. For the next step, the system may be further optimized considering both sensitivity, time, and cost, by comparing the performance of GNPR with ethanol and butanol in the mini-sensing system.

In the future, the current sensing system of the fluorometer, microfluidic flow control unit, display monitor and input keyboard may be put into a highly portable, all-inone device at a size of $30 \times 20 \times 15 \mathrm{~cm}$ (Fig. API-4). With disposable sensing chip and sample reagent containers, a four cardiac marker sensing may be performed withing 15 min in an emergency room or even in an emergency-medical-service vehicle.

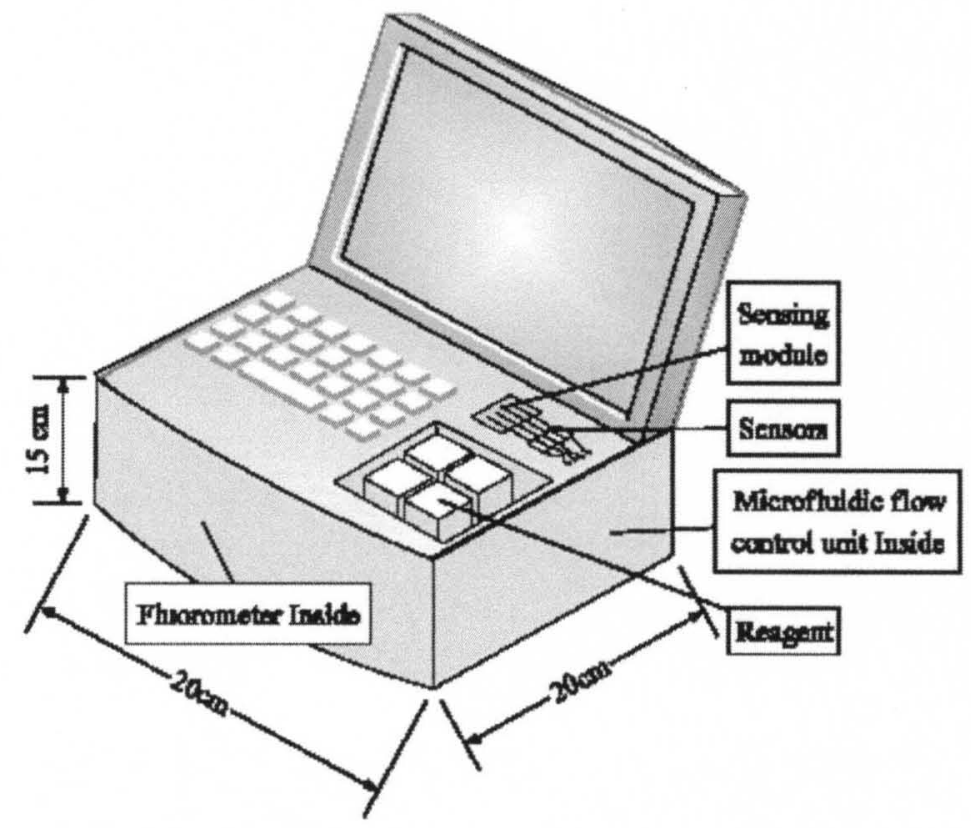

Figure API-4. Schematic design diagram of all-in-one four cardiac marker sensing device. 


\section{E. REFERENCE FOR APPENDIX-I}

1. Apple FS, Christenson RH, Valdes R, Andriak AJ, Mascotti K, and Wu AHB, Simultaneous Rapid Measurement of Whole Blood Myoglobin, Creatine Kinase $\mathrm{Mb}$, and Cardiac Troponin I by the Triage Cardiac Panel for Detection of Myocardial Infarction, Clinical Chemistry, 45, 199-205, 1999

2. Bernard R, Corday E and Eliasch H, Nomenclature and Criteria for Diagnosis of Ischemic Heart Disease. Report of the Joint International Society and Federation of Cardiology/World Health Organization Task Force on Standardization of Clinical Nomenclature, Circulation, 59, 607-609, 1979

3. Hong B and Kang KA, Biocompatible, Nanogold-Particle Fluorescence Enhancer for Fluorophore Mediated, Optical Immunosensor, Biosensors and Bioelectronics, $21,1333-1338,2006$

4. Hong B and Kang KA, Fluorescence Enhancers for Fluorophore Mediated Biosensors for Cardiovascular Disease Diagnosis, Advances in experimental medicine and biology, 578, 179-184, 2006

5. Hong B, Tang L, Ren YJ and Kang KA, Real-Time, Automated, Fluorophore Mediated Multi-Cardiac Marker Biosensing System with Nano-Metallic Particle Reagent, Advances in Experimental Medicine and Biology, 599, 23-29, 2008

6. Kang KA and Hong B, Biocompatible Nano-Metal Particle Fluorescence Enhancers Critical Reviews in Eukaryotic Gene Expression, 16, 45-60, 2006

7. Maisel AS, Krishnaswamy P, Herrmann HC and McCullough PA, Rapid Measurement of B-Type Natriuretic Peptide in the Emergency Diagnosis of Heart Failure, New England Journal of Medicine, 347, 161-167, 2002

8. Pope JH, Aufderheide TP and Ruthazer R, Missed Diagnoses of Acute Cardiac Ischemia in the Emergency Department, The New England Journal of Medicine $342,1167-1170,2000$

9. Sabatine MS, Morrow DA, Cannon CP and Braunwald E, Multimarker Approach to Risk Stratification in Non-St Elevation Acute Coronary Syndromes: Simultaneous Assessment of Troponin I, C-Reactive Protein, and B-Type Natriuretic Peptide, Circulation, 105, 1760-1763, 2002

10. Spiker JO, Kang KA, Drohan W and Bruley DF, Protein C Detection Via Fluorophore Mediated Immuno-Optical Biosensor, Advances in Experimental Medicine and Biology, 428, 621-627, 1999 
11. Tang L, Multi-Analyte, Fiber-Optic Immuno-Biosensing System for Rapid Disease Diagnosis: Model Systems for Anticoagulants and Cardiac Markers, Dissertation. Chemical Engineering, University of Louisville, Louisville, KY., 2005

12. Tang L and Kang KA, Preliminary Study of Simultaneous Multi-Anticoagulant Deficiency Diagnosis by a Fiber Optic Multi-Analyte Biosensor, Advances in Experimental Medicine and Biology, 566, 303-309, 2006

13. Tang L, Ren YJ, Hong B and Kang KA, A Fluorophore-Mediated, Fiber-Optic, Multi-Analyte, Immuno-Sensing System for Rapid Diagnosis and Prognosis of Cardiovascular Diseases, Journal of Biomedical Optics 11, 021011, 2006

14. Wu A, Apple FS, Gibler WB, Jesse RL, Warshaw MM and Valdes R, National Academy of Clinical Biochemistry Standards of Laboratory Practice: Recommendations for the Use of Cardiac Markers in Coronary Artery Diseases, Clinical Chemistry 45, 1104, 1999 


\section{APPENDIX II \\ HOLLOW GOLD NANOSPHERE FOR CONDITIONAL FLUORESCENCE QUENCHING}

\section{A. BACKGROUND}

Solid, spherical gold nanoparticles (GNPs) are known to generate surface plasmon field resonance around $520 \mathrm{~nm}$ [Eustics and El-Sayed, 2006]. Other gold nanostructures such as shells, thin films, nanorods, and triangles also generate strong surface plasmon field by the visible light, and the wavelength of their absorption peaks are strongly dependent on the dimension of their nanostructure [Hu, et al., 2006]. Dr. Jin Z. Zhang's group at the University of California, Santa Cruz, California, developed hollow gold nanospheres (HGNs) with various plasmon resonance peaks in the entire range of the visible and NIR wavelengths, by varying the diameter-to-wall thickness ratio [Hu, et al., 2006; Schwartzberg, et al., 2006].

HGNs tuned for NIR feature several advantages. Their strong absorption in NIR makes the HGN an effective absorption contrast agent for NIR imaging. For example, the extinction coefficient of hemoglobin, one of the strongest bio-chromophore in NIR, is in the range of $3 \times 10^{2} / \mathrm{cm} \mathrm{M}[\mathrm{Kim}$, et al., 2005]. From our measurement and calculation, the extinction coefficient of the HGNs that we currently use is in the range of 5-12 x $10^{10} / \mathrm{cm} \mathrm{M}$, which is approximately 8 orders of magnitude higher than that of hemoglobin. Because of this high absorption in NIR they were used for NIR 
hyperthermia very effectively [Ji, et al., 2007; Lu, et al., 2009]. GNPs are also strong scatterers for X-ray and therefore HGNs can be effective X-ray/CT contrast agents [Zhang, 2010]. These multiple properties allow HGNs be excellent candidates for both cancer detection (NIR fluorescence/X-ray/optical imaging) and treatment.

According to our theoretical analysis on fluorescence quenching by GNP [Chapter IV, section 1], when the Ex/Em wavelengths of a fluorophore are near the plasmon resonance wavelength of the particle, the fluorescence is strongly quenched. Therefore, HGNs with plasmon resonance peak in the far red or NIR region can be a highly effective quencher for NIR fluorophores.

Here, the conditional emission of Cypate fluorescence was studied utilizing HGNs, using the short spacer containing the -G-G-R- motif to be cleaved by uPA [Chapter IV, section 2]. HGNs studied were: (i) $\mathrm{HGN}_{654}$ : The particle has the outer diameter of $41 \mathrm{~nm}$ with the hollow center diameter of $28 \mathrm{~nm}(28 / 41 \mathrm{~nm})$. Its plasmon resonance peak is at $654 \mathrm{~nm}$; (ii) $\mathrm{HGN}_{786}$ : The hollow and outer diameter are 43 and 53 $\mathrm{nm}$, respectively, and the plasmon resonance peak is at $786 \mathrm{~nm}$.

\section{B. MATERIALS, INSTRUMENTS AND METHODS}

$\operatorname{HGN}_{654}\left(3.32 \times 10^{-6} \mu \mathrm{M}\right)$ and $\mathrm{HGN}_{786}\left(2.49 \times 10^{-6} \mu \mathrm{M}\right)$ stabilized with citric acid in water were provided by the Zhang group at the University of California, Santa Cruz. Cypate and Cypate conjugated short-spacer $\mathrm{HSCH}_{2} \mathrm{CH}_{2} \mathrm{NH}-\mathrm{G}-\mathrm{G}-\mathrm{R}-\mathrm{G}-\mathrm{G}-\mathrm{G}-\mathrm{NH}_{2}(s \mathrm{SP}-$ Cy), sPEG and uPA were as described in the materials section (Chapter III).

HGN-sSP-Cy was synthesized and purified using the same methods used for synthesizing GNP-sSP-Cy (Chapter III). For $\mathrm{HGN}_{654}$, the concentration of $\mathrm{HGN}_{654}$ and 
Cypate in the $\mathrm{HGN}_{654}-s \mathrm{SP}-\mathrm{Cy}$ was quantified by the absorption at 654 and $780 \mathrm{~nm}$, respectively. For $\mathrm{HGN}_{786}-\mathrm{SP}-\mathrm{Cy}$ because the absorption of $\mathrm{HGN}_{786}$ and Cypate significantly overlap, we were not able to quantify these by the spectroscopic method.

The fluorescence of the sample in 0.001M PBS buffer was measured in a 96-well Uniplate using a Spectra Gemini XPS fluorometer at the excitation and emission wavelengths of 780 and $830 \mathrm{~nm}$, respectively.

\section{RESULTS AND DISCUSSION}

\section{Fluorescence Quenching and Restoration of $\mathrm{HGN}_{654}-\mathrm{SSP}-\mathrm{Cy}$}

The fluorescence of $\mathrm{HGN}_{654}-\mathrm{SSP}-\mathrm{Cy}$ was measured and compared to that of the free $s \mathrm{SP}-\mathrm{Cy}$ at the same $s \mathrm{SP}-\mathrm{Cy}$ concentration $(5 \mu \mathrm{M})$; (Fig. 1). At this Cypate concentration the $\mathrm{HGN}_{654}$ concentration was $3.5 \times 10^{-3} \mathrm{nM}$.

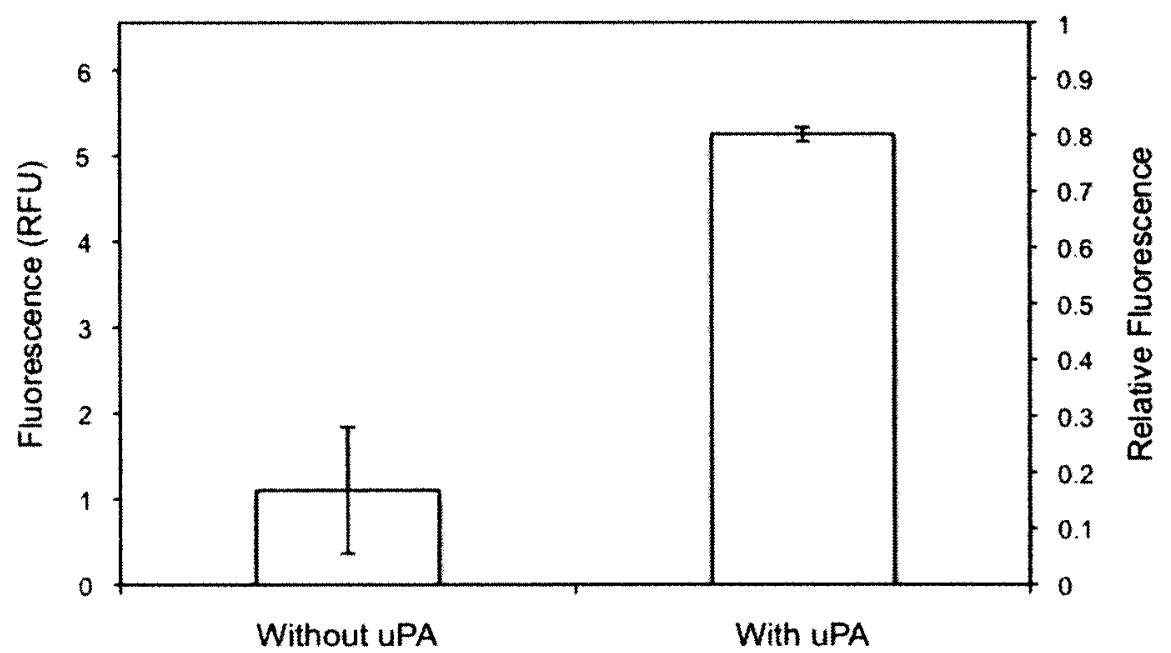

Figure APII-1. Absolute and relative fluorescence of $\mathrm{HGN}_{654}-5 \mathrm{SP}-\mathrm{Cy}$, before and after adding uPA (30 min after uPA addition). [Experiment conditions: Cypate conc., $5 \mu \mathrm{M}$; HGN conc., $3.5 \times 10^{-3} \mathrm{nM}$; Ex/Em 780/830 $\mathrm{nm}]$ 
In $\mathrm{HGN}_{654}-\mathrm{SSP}-\mathrm{Cy}$ the Cy fluorescence was quenched by $84 \%$. Compared to $s \mathrm{SP}-$ Cy with 3.7 or $8 \mathrm{~nm}$ GNPs (with GNP concentration 31 or $7 \mathrm{nM}$, quenched by 96 or $95 \%$, respectively) this was slightly less.

After uPA was added (1030 unit/mL, at a sufficient amount) to the $\mathrm{HGN}_{654}-\mathrm{sSP}$ Cy sample, the fluorescence was restored to $80 \%$ of the free Cypate.

\section{Fluorescence Quenching and Restoration of $\mathrm{HGN}_{786}-\mathrm{sSP}-\mathrm{Cy}$}

The absorption peak $(786 \mathrm{~nm})$ of $\mathrm{HGN}_{786}$ is very close to the emission wavelength of Cypate $(830 \mathrm{~nm})$. $\mathrm{HGN}_{786}$ and Cypate in the final product $\left(\mathrm{HGN}_{786}-\mathrm{SP}\right.$ Cy) could not be determined because the HGN absorption peak highly overlaps with that of Cypate. Therefore, only the absolute fluorescence before and after adding uPA were obtained (Fig. 2), without the relative fluorescence values.

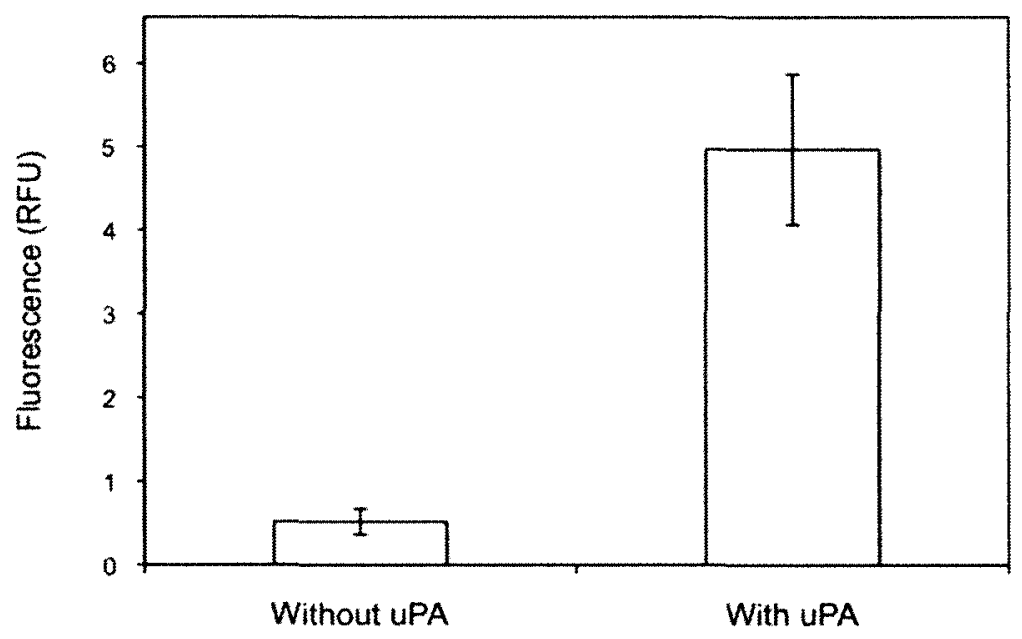

Figure APII-2. Fluorescence of $\mathrm{HGN}_{786}-\mathrm{SSP}-\mathrm{Cy}$ before and after adding uPA (30 min after uPA addition), with Ex/Em 780/830 nm. 
The fluorescence of $\mathrm{HGN}_{786}$-SP-Cy without uPA was $0.52 \mathrm{RFU}$, at the lower limit of the instrument, suggesting that the Cypate fluorescence was almost or completely quenched by $\mathrm{HGN}_{786}$. When uPA was added to the sample, the fluorescence increased to 5.0 RFU. Assuming that both $\mathrm{HGN}_{654}$ and $\mathrm{HGN}_{786}$ have similar restoration rate, the fluorescence of $\mathrm{HGN}_{786}-\mathrm{SP}-\mathrm{Cy}$ is $53 \%$ lower than that of $\mathrm{HGN}_{654}-\mathrm{SP}-\mathrm{Cy}$, and $\mathrm{HGN}_{786}$ appears to quench more effectively than $\mathrm{HGN}_{654}$ does, due to its stronger absorption at the Cypate emission peak's wavelength.

\section{CONCLUSIONS}

The two HGNs, which absorption wavelengths are adjusted in NIR, especially $\mathrm{HGN}_{786}$, demonstrated an excellent ability for quenching Cypate fluorescence when Cypate is conjugated on them via the short spacer. The conditional fluorescence emission in the presence of the cancer-secreting enzyme was well confirmed. This conditional emission property is especially important because it adds an imaging-contrast ability to the nano-entity that already possesses multi-functions of NIR absorption contrast; X-ray scattering contrast; and photo-thermal property.

\section{E. REFERENCE FOR APPENDIX II}

1. Eustics S and El-Sayed MA, Why Gold Nanoparticles Are More Precious Than Pretty Gold: Noble Metal Surface Plasmon Resonance and Its Enhancement of the Radiative and Non-Radiative Properties of Nanocrystals of Different Shapes, Chemical Society Reviews, 35, 209-217, 2006

2. Hu M, Chen J, Li Z-Y, Au L, Hartland GV, Li X, Marquez M and Xia Y, Gold Nanostructures: Engineering Their Plasmonic Properties for Biomedical Applications, Chemical Society Reviews, 35, 1084-1094, 2006 
3. Ji X, Shao R, Elliott AM, Stafford RJ, Esparza-Coss E, Bankson JA, Liang G, Luo ZP, Park K, Markert JT and Li C, Bifunctional Gold Nanoshells with a Superparamagnetic Iron Oxide-Silica Core Suitable for Both $\mathrm{Mr}$ Imaging and Photothermal Therapy, Journal of Physical Chemistry C, 111, 6245-51, 2007

4. Kim J, Xia M, Liu H, Extinction coefficients of hemoglobin for near-infrared spectroscopy of tissue. Engineering in Medicine and Biology Magazine, IEEE, $24,118-21,2005$

5. Lu W, Xiong CY, Zhang GD, Huang Q, Zhang R, Zhang JZ and Li C, Targeted Photothermal Ablation of Murine Melanomas with Melanocyte-Stimulating Hormone Analog-Conjugated Hollow Gold Nanospheres, Clinical cancer research, 15, 876-886, 2009

6. Schwartzberg AM, Olson TY, Talley CE and Zhang JZ, Synthesis, Characterization, and Tunable Optical Properties of Hollow Gold Nanospheres, Journal of Physical Chemistry B, 110, 19935-44., 2006

7. Zhang JJ, Biomedical Applications of Shape-Controlled Plasmonic Nanostructures: A Case Study of Hollow Gold Nanospheres for Photothermal Ablation Therapy of Cancer, The Journal of Physical Chemistry Letters, 1, 686695,2010 


\section{CURRICULUM VITAE}

NAME: Jianting Wang

ADDRESS: $\quad$ Department of Chemical Engineering

Speed School of Engineering, University of Louisville

Louisville, KY 40292

DOB:

Xi'an, Shaanxi, P. R. China; January 12, 1983

EDUCATION: $\quad$ B.S. in Chemical Engineering

Tsinghua University

1999-2003

M.S. in Chemical Engineering

Tsinghua University

2003-2006

Ph.D. in Chemical Engineering

University of Louisville

2006- 2011

AWARDS/

The University Graduate Fellowship, Graduate school

HONORS:

University of Louisville, 2006-2008

Travel Award, Institute for Molecular Diversity and Drug Design, Univ. of Louisville, 2007 Annual AIChE conference, Salt Lake City, Utah, USA, Nov. 4-6, 2007

The Britton Chance award for young researcher for student, International Society on Oxygen Transport to Tissue annual conference, 2008

Travel Award, Institute for Molecular Diversity and Drug Design, Univ. of Louisville, 2008 annual AIChE conference, Philadelphia, PA, Nov. 17-21, 2008

The engineering collaboration award for graduate student, First Place, Research!Louisville 2009

Duane F. Bruley travel awards for student, International Society on Oxygen Transport to Tissue annual conference, 2009

E-Expo research competition award, 5th Place, Expo, Speed school, University of Louisville, 2010 
Arno Spatola graduate research award, The Institute for Molecular Diversity and Drug Design $\left(\mathrm{IMD}^{3}\right)$, University of Louisville, 2010 $-2011$

PROFESSIONAL American Institute of Chemical Engineering (AIChE)

SOCIETIES: $\quad$ Biomedical Engineering Society (BMES)

International Society on Oxygen Transport to Tissue (ISOTT)

\section{PUBLICATIONS:}

1. J. Wang, J. Moore, S. Laulhe, M. Nantz, S. Achilefu, K. A. Kang' Fluorophore-gold nanoparticle complex for sensitive optical biosensing and imaging, Submitted

2. J. Wang, D. Wheeler, J. Z. Zhang, S. Achilefu, and K. A. Kang, NIR fluorophore-gold nanosphere complex for cancer enzyme triggered detection and hyperthermia, Proceeding of ISOTT annual meeting 2011, Jul. 25-28, Washington, DC, 2011

3. K. A. Kang, J. Wang, M. O'Toole, J.D. Moore, S. Laulhe, M. Nantz, S. Achilefu, Sensitivity Enhancement of NIR Fluorescence Contrast Agent Utilizing Gold Nanoparticle, Advances in Experimental Medicine and Biology, 737: 285-291 (in print)

4. J. Wang, M. Nantz, S. Achilefu, K. A. Kang. Gold nanoparticle-fluorophore complex for conditionally fluorescing signal mediator, Analytica Chimica ACTA, 696:96-104, 2011

5. K. A. Kang, J. Wang, J. B. Jasinski, S. Achilefu, Fluorescence Manipulation by Gold Nanoparticles: From Complete Quenching to Extensive Enhancement, Journal of Nanobiotechnology, 9:16, 2011

6. J. Wang, M. O'Toole, A. Massey, S. Biswas, M. Nantz, S. Achilefu, K. A. Kang. Highly specific, NIR fluorescent contrast agent with emission controlled by gold nanoparticle, Advances in Experimental Medicine and Biology, 701:149-54, 2011

7. J. Wang, M. Nantz, S. Achilefu, K. A. Kang. FRET-like fluorophorenanoparticle complex for highly specific cancer localization, Advances in Experimental Medicine and Biology, 662:407-13 
8. J. Wang, B. Hong, J. Kai, J. Han, Z. Zou, C. H. Ahn, and K. A. Kang. Mini sensing chip for Point-of-Care AMI diagnosis utilizing MEMS and nanotechnology, Advances in Experimental Medicine and Biology, 645:101-107, 2009.

9. J. Wang, D. Chen, and S. Hu, Planning decision for a natural gas chemical company by map of decision network, Journal of Chemical Industry and Engineering (China), 59:2289-2294, 2008

10. J. Wang, S. Hu, Y. Li, and D. Xue, Industrial planning decision with consideration of environmental effects, Modern Chemical Industry, 25:58-62, 2005

11. D. Zheng, S. Hu, Y. Li, J. Shen, and J. Wang, Mass integration for ecoindustrial parks, Computers and Applied Chemistry, 21:6-10, 2004

12. J. F. Wang, W. Ma, S. Hu, H. Luo, J. Shen, Y. Li, and J. Wang, Planning of changsha huangxing eco-industrial park, Computers and Applied Chemistry, $21: 48-50,2004$

\section{NATIONAL/INTERNATIONAL MEETING PRESENTATIONS:}

1. J. Wang, M. Nantz, S. Achilefu, J. Z. Zhang, and K. A. Kang, Highly Sensitive, Optical Contrast Agent with Gold Nanoparticle and Hollow Gold Nanophere, Biomedical Engineering Society 2011 Annual Fall Meeting, Hartford, CT, Oct. 12-15, 2011 (Poster, to be presented)

2. J. Wang, M. Nantz, S. Achilefu, J. Z. Zhang, and K. A. Kang, Tailored fluorescence emission level of optical contrast agent by gold nanoparticles, 2011 annual AIChE conference, Minneapolis, MN, Oct. 16-21, 2011 (Oral, to be presented)

3. J. Wang, D. Wheeler, J. Z. Zhang, S. Achilefu, and K. A. Kang, NIR fluorophore-gold nanosphere complex for cancer enzyme triggered detection and hyperthermia, ISOTT annual meeting, Jul. 25-28, Washington, DC, 2011 (Oral)

4. J. Wang, B. Souvik, J.D. Moore, S. Laulhe, M. Nantz, S. Achilefu, and K. A. Kang, Enzyme triggered, nanoparticle controlled fluorescence emission for sensitive and specific breast cancer detection, 2010 annual AIChE conference, Salt lake city, UT, Nov. 7, 2010 (Oral)

5. J. Wang, B. Souvik, M. Nantz, S. Achilefu, and K. A. Kang, Development of cancer enzyme triggered fluorescent nano-contrast agent, Biomedical 
Engineering Society 2009 Annual Fall Meeting, Austin, TX, Oct. 6, 2010 (Oral)

6. K. A. Kang, J. Wang, M. O'Toole, J.D. Moore, S. Laulhe, M. Nantz, S. Achilefu, Sensitivity Enhancement of NIR Fluorescence Contrast Agent Utilizing Gold Nanoparticle, ISOTT annual meeting, Jul. 18-23, Ascona, Switzerland, 2010 (Oral)

7. J. Wang, M. O'Toole, S. Achilefu, and K. A. Kang, Fluorescence level manipulation by gold nano particles, 2009 annual AIChE conference, Nashville, TN, Nov. 12, 2009 (Oral)

8. M. O'Toole, J. Wang, K. Sanapala, J. Jasinski, and K. A. Kang, Gold-coated iron-oxide nanoparticles for optical imaging and hyperthermia of cancer, 2009 annual AIChE conference, Nashville, TN, Nov. 12, 2009 (Oral)

9. J. Wang, M. O'Toole, J. Jasinski, S. Achilefu, M. Nantz, and K. A. Kang, From quenching to enhancement - Fluorescence emission manipulation using gold nano particles, Biomedical Engineering Society 2009 Annual Fall Meeting, Pittsburgh, PA, Oct. 8, 2009 (Poster)

10. M. O'Toole, J. Wang, K. Sanapala, J. Jasinski, and K. A. Kang, A single nanoentity for optical contrast enhancement and hyperthermia treatment, Biomedical Engineering Society 2009 Annual Fall Meeting, Pittsburgh, PA, Oct. 8, 2009 (Oral)

11. J. Wang, M. O’Toole, A. Massey, S. Biswas, M. Nantz, S. Achilefu, K. A. Kang. Highly specific, NIR fluorescent contrast agent with emission controlled by gold nanoparticle, ISOTT annual meeting, Jun. 5-9, Cleveland, $\mathrm{OH}, \mathrm{USA}$ (Oral)

12. J. Wang, S. Achilefu, K. A. Kang. Fluorescence emission control by nanometal particles, 2008 annual AIChE conference, Philadelphia, PA, Nov. 20, 2008 (Oral)

13. K. A. Kang, J. Wang, M. Nantz, S. Achilefu. Novel design of fluorophorenanoparticle complex for highly specific cancer locator, 2008 annual AIChE conference, Philadelphia, PA, Nov. 20, 2008 (Oral)

14. J. Wang, M. Nantz, S. Achilefu, K. A. Kang. Controlled fluorescence emission in molecular sensing/imaging by nanometal particles, Biomedical Engineering Society 2008 Annual Fall Meeting, St. Louis, USA, Oct. 4, 2008 (Poster)

15. K. A. Kang, J. Wang, M. Nantz, S. Achilefu. Highly Specific Molecular Recognition Via Conditional Fluorescence Emission by Nanometal Particles, 
Biomedical Engineering Society 2008 Annual Fall Meeting, St. Louis, USA, Oct. 3, 2008 (Oral)

16. J. Wang, M. Nantz, S. Achilefu, K. A. Kang. FRET-like fluorophorenanoparticle complex for highly specific cancer localization, 36th ISOTT annual meeting, Aug. 7, Sapporo, Japan, 2008 (Oral)

17. J. Wang, B. Hong, J. Kai, J. Han, Z. Zou, C. H. Ahn, and K. A. Kang, Nanometal particle reagent for highly sensitive, real-time, fluorophore mediated biosensor, 2007 annual AIChE conference, Salt Lake City, Utah, USA, Nov. 5, 2007 (Oral)

18. J. Wang, B. Hong, H. Jin and K. A. Kang. Nano-metal particles for fluorescence enhancement in fluorophore mediated biosensing and bioimaging, 2007 annual AIChE conference, Salt Lake City, Utah, USA, Nov. 5, 2007 (Oral)

19. J. Wang, B. Hong, J. Kai, J. Han, Z. Zou, C. H. Ahn, and K. A. Kang. Nanogold particle reagent and MEMS for highly sensitive, automatic, multicardiac marker sensing, Biomedical Engineering Society 2007 Annual Fall Meeting, Los Angels, USA, Sep. 28, 2007 (Poster)

20. J. Wang, B. Hong, J. Kai, J. Han, Z. Zou, C. H. Ahn, and K. A. Kang. Mini sensing chip for Point-of-Care AMI diagnosis utilizing MEMS and nanotechnology, The 35th ISOTT annual meeting, Uppsala, Sweden, Aug. 28, 2007 (Oral)

21. J. Wang, S. Hu, Y. Li, and D. Xue, Industrial planning decision with green efficient index, International Society for Industrial Ecology Conference, Stockholm, Sweden, Jun. 15, 2005 (Poster)

\section{LOCAL/INSTITUTIONAL PRESENTATIONS}

1. J. Wang, M. O'Toole, S. Biswas, J. D. Moore, S. Laulhe, M. Nantz, S. Achilefu, J. Z. Zhang and K. A. Kang, Nano-light bulb for breast cancer detection E-Expo 2011, Louisville, KY, Mar. 5, 2011 (Poster)

2. J. Wang, M. O'Toole, S. Biswas, J. D. Moore, S. Laulhe, M. Nantz, S. Achilefu, J. Z. Zhang and K. A. Kang, Gold nanoparticle based, fluorescent contrast agent for highly specific and sensitive disease detection and diagnosis, Institute for Molecular Diversity and Drug Design Symposium, Louisville, KY, Mar. 8, 2011 (Poster)

3. J. Wang, R. Lupitskyy, M. Nantz, S. Achilefu, and K. A. Kang, Enzymetriggered, gold nanoparticle-based optical contrast agent for breast cancer 
detection, the 9th Annual Brown Cancer Center Retreat, Louisville, KY, Nov. 5, 2010 (Poster)

4. J. Wang, M. O'Toole, S. Biswas, M. Nantz, S. Achilefu, and K. A. Kang, Enzyme-triggered fluorescent contrast agent for highly specific breast cancer detection, Institute for Molecular Diversity and Drug Design Symposium, Louisville, KY, Mar. 9, 2010 (Poster)

5. J. Wang, M. O'Toole, M. Nantz, S. Achilefu, and K. A. Kang, Nano-light bulb locating breast cancer, E-Expo 2010, Louisville, KY, Mar. 6, 2010 (Poster)

6. J. Wang, M. O'Toole, S. Biswas, M. Nantz, S. Achilefu, and K. A. Kang, Molecular beacon-like nano contrast agent for early breast cancer detection, the 8th Annual Brown Cancer Center Retreat, Louisville, KY, Nov. 6, 2009 (Poster)

7. J. Wang, M. O'Toole, S. Biswas, M. Nantz, S. Achilefu, and K. A. Kang, Molecular beacon-like nano contrast agent for highly specific breast cancer imaging, 2009 Institute for Molecular Diversity and Drug Design Fall Fest, Louisville, KY, Oct. 29, 2009 (Oral)

8. J. Wang, M. O'Toole, Souvik Biswas, M. Nantz, S. Achilefu, and K. A. Kang, Molecular beacon-like fluorophore-gold nanoparticle complex for highly specific breast cancer imaging, Research!Louisville, Louisville, KY, Oct. 13, 2009 (Poster)

9. J. Wang, M. O'Toole, M. Nantz, and K. A. Kang, Molecular beacon-like nano-entity for highly specific breast cancer detection, Institute for Molecular Diversity and Drug Design Symposium, Louisville, KY, Mar. 10, 2009 (Poster)

10. J. Wang, M. O'Toole, M. Nantz, S. Achilefu, and K. A. Kang, Tiny Particle, Big Future - Nanotechnology in disease diagnosis and treatment, E-Expo 2009, Louisville, KY, Mar. 7, 2009 (Poster)

11. J. Wang and K. A. Kang, Application of nanometal particles in biosensing, bioimaging and cancer treatment, Graduate Research Day at the Capitol, Frankfort, KY, Feb.2, 2009 (Poster)

12. J. Wang, M. Nantz, S. Achilefu, and K. A. Kang, Artificial fluorescence alteration by nanometal particle in targeted biosensing and molecular imaging, Research!Louisville, Louisville, KY, Oct. 16-19, 2008 (Poster) 
13. J. Wang, B. Hong, J. Kai, J. Han, Z. Zou, C. H. Ahn, and K. A. Kang, M. H. Nantz, S. S. Kakar, and K. A. Kang, Big future in medicine by tiny particles: nano-metal particles for disease diagnosis and treatment, Ohio Valley Affiliates for Life Sciences $6^{\text {th }}$ Annual Conference, Louisville, KY, Apr. 14, 2008 (Poster)

14. J. Wang, B. Hong, J. Kai, J. Han, Z. Zou, C. H. Ahn, and K. A. Kang. Nanogold particle reagent and MEMS for highly sensitive, automatic, multicardiac marker sensing, KYNANOMAT workshop, Louisville, KY, Mar. 1618, 2008 (Poster)

15. J. Wang, B. Hong, J. Kai, J. Han, Z. Zou, C. H. Ahn, and K. A. Kang, M. H. Nantz, S. S. Kakar, and K. A. Kang, Application of nano-metal particles in biosensing, bioimaging, and cancer treatment, 2008 Institute for Molecular Diversity and Drug Design Symposium, Louisville, KY, Mar. 11, 2008 (Poster)

16. J. Wang, B. Hong, J. Kai, J. Han, Z. Zou, C. H. Ahn, and K. A. Kang, M. H. Nantz, S. S. Kakar, and K. A. Kang, Application of nano-metal particles in disease diagnosis and treatment, E-Expo 2008, Louisville, KY, Mar. 1, 2008 (Poster)

17. J. Wang, B. Hong, J. Kai, J. Han, Z. Zou, C. H. Ahn, and K. A. Kang, Application of nano and MEMS technologies in the development of mini sensing chip for real-time AMI diagnosis, Research!Louisville, Louisville, KY, Oct. 16-19, 2007 (Poster) 